\title{
Single Mode Excitation in the Shallow Water Acoustic Channel Using Feedback Control
}

by

\author{
John R. Buck \\ Submitted in partial fulfillment \\ of the requirements for the degree of \\ Doctor of Philosophy \\ at the \\ Massachusetts Institute of Technology \\ and the
}

$1 \cdot \mathrm{O} \cdot \mathrm{H} \cdot \mathrm{M}$ 'SSWW' $370 \mathrm{H}$ SO00\%

1 d\&y 7 1.80178069? $780150 \% 50$ Jus $\therefore$ its

Woods Hole Oceanographic Institution

June 1996

(c) John R. Buck, MCMXCVI. All rights reserved.

The author hereby grants to MIT and to WHOI permission to reproduce and to distribute copies of this thesis document in whole or in part.

Author

MIT Department of EECS and the MIT/XyHOI Joint Program in Oceanography and Oceanographic Engineering, May 23, 1996

Certified by James C. Preisig

Visiting Investigator, WHOI, Thesis Supervisor

Certified by

Alan V. Oppenheim

Distinguished Professor of Electrical Engineering, MIT, Thesis Supervisor

Accepted by

Henrik Schmidt Acting Chairman, MIT/WHOI Joint_Gommiftee, on Oceanographic Engineering Accepted by

Frederic R. Morgenthaler Chairman, MIT EECS' Departmental Commirtee on Graduate Students 


\title{
Single Mode Excitation in the Shallow Water Acoustic Channel Using Feedback Control
}

\author{
by \\ John R. Buck \\ Submitted in partial fulfillment of the \\ requirements for the degree of \\ Doctor of Philosophy \\ at the \\ MASSACHUSETTS INSTITUTE OF TECHNOLOGY \\ and the \\ WOODS HOLE OCEANOGRAPHIC INSTITUTION \\ June 1996
}

\begin{abstract}
The shallow water acoustic channel supports far-field propagation in a discrete set of modes. Ocean experiments have confirmed the modal nature of acoustic propagation, but no experiment has successfully excited only one of the suite of mid-frequency propagating modes propagating in a coastal environment. The ability to excite a single mode would be a powerful tool for investigating shallow water ocean processes. A feedback control algorithm incorporating elements of adaptive estimation, underwater acoustics, array processing and control theory to generate a high-fidelity single mode is presented. This approach also yields a cohesive framework for evaluating the feasibility of generating a single mode with given array geometries, noise characteristics and source power limitations. Simulations and laboratory waveguide experiments indicate the proposed algorithm holds promise for ocean experiments.
\end{abstract}

Thesis Supervisor: Alan V. Oppenheim

Title: Distinguished Professor of Electrical Engineering, MIT

Thesis Supervisor: James C. Preisig

Title: Visiting Investigator, WHOI 


\section{Acknowledgments}

First, and most importantly, I want to thank my parents and family: Dad, Mom, Dul, Jose, Rich, and Slick. Without their love, support and encouragement, I would not have been in a position to attempt this work, let alone complete it. This is a debt beyond the possibility of repayment.

$\mathrm{Al}$ Oppenheim has been an invaluable mentor for the last eight years. Both by example and advice, $\mathrm{Al}$ has taught me a tremendous amount about how to be a teacher, scientist, writer, and mentor, giving me room to do these things in my own way, while challenging me to do them all as well as I possibly could. The chance that led me into his class as a junior has been one of the most fortunate things that has happened to me in my life. I look forward to continuing our friendship for many years.

Without Jim Preisig's advice and encouragement, the work in this thesis would be a poor shadow of what it is now. His valuable technical feedback and suggestions on my work has consistently led me to fruitful new approaches. More importantly, Jim has graciously navigated the transition from fellow grad student to advisor in a way that never once strained our friendship. Jim's intellectual generosity is unrivaled in my experience. I appreciate the time and understanding he has given me over the last four years.

I want to thank my committee members, Art Baggeroer and Jim Lynch, for keeping my work solidly anchored in the realities of oceanography. I have benefited greatly from their combined years of ocean-going experience and the important suggestions and critiques of my ideas.

Mark "The Hawk" Johnson played the part of the Fifth Business in this thesis. Although he had no official academic role, Mark was absolutely essential in getting this work completed. His unflagging enthusiasm and conviction this work was good science and should be done helped me through several rough spots. The success of the experimental work in Chapter 4 is a testimony to his technical expertise and dedication. I will not soon forget many Hawk Feeds we shared during the winter months in Woods Hole.

The Digital Signal Processing Group at MIT has been a great environment for me during my graduate student career. I have greatly enjoyed sharing conversations, ideas, laughter, and of course, meals, with my fellow group members. I feel especially indebted to Steve Isabelle, Denis Peregrym, Andy Singer, Kathleen Wage, and Greg Wornell. I have been truly blessed to have companions such as these. I also want to thank Giovanni Aliberti, Maggie Beucler and Sally Santiago for smoothing out many of the rough spots along the way. I am at a loss to say what I would have done without their patient and expert assistance.

I have benefitted from conversations with many faculty in several departments both at MIT and WHOI during this research. George Frisk, Henrik Schmidt, George Verghese, and Alan Willsky patiently listened to me fumbling about in fields I knew 
little about and tactfully guided me towards a better understanding of what I was trying to do.

The experimental work in this thesis is the result of the active assistance of several people at WHOI. Matt Grund's help with the computer system in general and AMS specifically was invaluable. Matt's good nature and humor helped keep me on an even keel through some long winter weeks in Woods Hole. Karlen Wannop's work preparing the electronics for the flume went far above and beyond the call of duty. Keith Von der Heydt gave helpful advice and remarkably trusting loans of equipment on several occasions. Stan Rosenblad and Subramaniam Rajan loaned us the sources for the experiment. Don Peters accomodated our eratic experimental schedule. Vector Research kindly loaned us the sound damping plates. John Kemp made the lead ballast for the hydrophone array. Matt Gould generously loaned us his prodigious strength and mechanical expertise in setting up the flume.

The Department of Applied Ocean Physics and Engineering at WHOI has also been a productive and enjoyable second home for me. I want to thank my fellow Joint Program students, Joe Bondaryk, Trym Eggen, Brian Sperry, Brian Tracey, and Pete Traykovski for their friendship and advice, not to mention phone messages, over many summers. Ann Henry in the AOPE department and Abby Alvin, Jake Peirson and John Farrington have expertly and efficiently solved all manner of problems and crises for me.

Many friends in Boston and on the Cape have helped me survive and even thrive during my time in graduate school. I especially want to thank Susan Alderman for her love, encouragement, and for generously giving me the time I needed to write over the past year. The denizens of Decomoronoanthropolis, Cork, Dan, Doug, Erika, James, Jon, Kelly, Kevin, Marc, Mike, Nick, Pat, Sean, and Tim have all been great friends and housemates, making a crazy idea a great if sometimes exasperating place to live. Outside the legions of dumb guys, Kirk, Heather, Linda, Sarah, Benson, Liz, and Erik have also been tried and true friends, sharing triumphs and tragedies. Gian-Carlo and Theo have time and again soothed my doubts and fears with sage advice. In Woods Hole, Dave, Amy, Jay, Jamie, Diane, and BillBill have welcomed me during the itinerant phases of the last several years. I have been fortunate beyond telling to have had such generous and true friends.

Josko Catipovic funded my research for summer of 1992 on the Office of Naval Research Grant Number N00014-92-J-1661 and from June 1993 through August 1995 on Defense Advanced Research Projects Agency Grant Number MDA972-92-J1041. The Office of Naval Research Grant N00014-95-1-0362 to MIT supported the computer facilities used to do much of this work.

This thesis is dedicated to the memory of Antonio Pizzigati. Tony was a true friend and a giant among men. The passion and energy he brought to everything he did inspired those of us fortunate enough to have known him. His kindness and compassion were without bound or equal in my experience.

Finally, as always, Ad Majorem Dei Gloriam. 
Write down the vision

Clearly upon the tablets, so that one can read it readily.

For the vision still has its time, presses on to fulfillment, and will not disappoint; If it delays, wait for it, it will surely come, it will not be late.

Habakuk 2:1-2 


\section{Contents}

1 Introduction $\quad 13$

1.1 Normal Mode Model for Acoustic Propagation . . . . . . . . . . . . . 17

1.2 Green's Function . . . . . . . . . . . . . . . . . 24

1.3 Mode Filters . . . . . . . . . . . . . . . . . . . . . 26

1.3.1 Sampled Mode Shapes Mode Filter . . . . . . . . . . . . . 27

1.3.2 Pseudo-Inverse Mode Filter . . . . . . . . . . . . . . . . . . 31

1.3.3 Diagonal Weighting . . . . . . . . . . . . . . . 33

1.3.4 Maximum A Posteriori Mode Filters . . . . . . . . . . . . . . 34

1.3.5 Relation of Mode Filters to Target Pressure Vector . . . . . . 37

1.4 Control Theory . . . . . . . . . . . . . . . . . . . . . . . . . . . . . . . . . . . . . .

1.5 Matched Signals . . . . . . . . . . . . . . . . . . . . . . . . . . . . . . . . . . . . 43

1.6 Summary . . . . . . . . . . . . . . . . . . 45

2 Control Algorithm $\quad 47$

2.1 Propagation Model . . . . . . . . . . . . . . . . . . . 49

2.2 Source Array Weight Selection . . . . . . . . . . . . . . . . . . . . . 52

2.3 Condition Number-limited Least Squares . . . . . . . . . . . . . . . . 58

2.4 Kalman Filter Estimator . . . . . . . . . . . . . . . . . . . . . . 63

2.5 Least Mean Squares Estimator . . . . . . . . . . . . . . . . . . . . 67

3 Simulation Results $\quad 69$

3.1 Range-Invariant Environment . . . . . . . . . . . . . . . . 70

3.2 Rock Outcropping . . . . . . . . . . . . . . . . . . . . 77

3.3 Downsloping Wedge . . . . . . . . . . . . . . . . . . . . . . . . . 84

3.4 Solitary Internal Wave . . . . . . . . . . . . . . . . . . . . . . . . . . . . . . . . . . . . . . .

3.5 Summary . . . . . . . . . . . . . . . . . . . 92

4 Laboratory Waveguide Experiments $\quad 95$

4.1 Waveguide and Equipment Description . . . . . . . . . . . . . . 96

4.2 Acoustic Properties of the Laboratory Waveguide . . . . . . . . . . . 98

4.3 Time-Invariant Waveguide . . . . . . . . . . . . . . . . . . . . 101

4.4 Time-Varying Channels . . . . . . . . . . . . . . . . . . . . . 109 
4.4.1 Abrupt Channel Change . . . . . . . . . . . . . . . . 110

4.4 .2 Gradual Channel Change . . . . . . . . . . . . . . . . . . 111

4.5 Summary . . . . . . . . . . . . . . . 116

5 Conclusions and Future Directions 117

A Transient Response of the CNLLS Estimator 123 


\section{List of Figures}

1-1 Proposed Experimental Setup . . . . . . . . . . . . . . . . . 15

1-2 Open-loop Control For Single Mode Excitation . . . . . . . . . . . . 39

1-3 Closed-loop Control For Single Mode Excitation . . . . . . . . . . . 39

2-1 Control Algorithm for Ocean Experiment . . . . . . . . . . . 48

3-1 Hydrographic Transect Providing Environmental Data for Simulations 70

3-2 Vertical Profile of Range-Invariant Environment . . . . . . . . . . . 71

3-3 Mode Shapes for Range-Invariant Environment . . . . . . . . . . 72

3-4 Ratio of Energy in Desired Profile to Energy in Mean Error for CNLLS Estimator . . . . . . . . . . . . . . . . 74

3-5 SER for CNLLS Estimator at different SNR levels . . . . . . . . . 75

3-6 Vertical Profile of Pressure for Typical Trial of CNLLS Estimator . . 75

3-7 Ratio of Energy in Desired Profile to Energy in Mean Error for Kalman Filter Estimator with $\alpha=0.98 \ldots \ldots$. . . . . . . . . 77

3-8 Ratio of Energy in Desired Profile to Energy in Mean Error for Kalman Filter Estimator with $\alpha=0.99 \ldots \ldots \ldots \ldots$. . . . . . 78

3-9 Ratio of Energy in Desired Profile to Energy in Mean Error for Kalman Filter Estimator with $\alpha=1.0 \ldots \ldots \ldots 78$

3-10 Vertical Profile of Pressure for Typical Trial of Kalman Filter Estimator with $\alpha=0.99, \mathbf{P}_{\mathbf{f}}=10^{-6} \mathbf{I} \ldots \ldots \ldots \ldots \ldots$

3-11 Comparison of CNLLS and Kalman filter estimators . . . . . . . . 79

3-12 Rock Outcropping Environment . . . . . . . . . . . . . . . . 81

3-13 Performance of Estimators For Rock Outcropping Environment . . . 82

3-14 Vertical Profile of Pressure for a Typical Trial in Rock Outcropping Environment . . . . . . . . . . . . . . . . . . 83

3-15 Pressure Field Excited by Open Loop Controller in Rock Outcropping Environment . . . . . . . . . . . . . . . . . 85

3-16 Pressure Field Excited by Feedback Controller in Rock Outcropping Environment . . . . . . . . . . . . . . . . 85

3-17 Downsloping Wedge Environment. . . . . . . . . . . . . . . . 86

3-18 Mean SER Convergence for Downsloping Wedge Environment . . . . 88

3-19 Typical Vertical Pressure Profiles for Downsloping Wedge Environment 88 
3-20 Sound speed profile $c(r, z)$ for solitary internal wave with $\operatorname{sech}^{2}$ profile 90

3-21 Performance of feedback control algorithm using CNLLS estimator compared with open loop controller for solitary wave propagating through feedback volume. . . . . . . . . . . . . . . . . . 91

3-22 Performance of feedback control using Kalman filter estimator compared with open loop controller for solitary wave propagating through the feedback volume. . . . . . . . . . . . . . . . . 91

4-1 Configuration of Laboratory Waveguide Experiments . . . . . . . 98

4-2 Singular Values of Replica Matrix for Laboratory Waveguide . . . . 100

4-3 Pressure Magnitude for System Modes of Laboratory Waveguide . . . 101

4-4 SER Performance for System Mode 1 in Time-Invariant Waveguide . 103

4-5 SER Performance for System Mode 2 in Time-Invariant Waveguide . 104

4-6 SER Performance for System Mode 3 in Time-Invariant Waveguide . 104

4-7 SER Performance for System Mode 4 in Time-Invariant Waveguide . 105

4-8 SER Performance for Mode $1+0.4 j$ Mode 2 in Time-Invariant Waveguide . . . . . . . . . . . . . . . . . . 105

4-9 Comparison of the SER for System Mode 1 in the Time-Invariant Waveguide for different $\mathbf{P}_{\mathbf{f}}$ in the Kalman Filter Channel Estimator . 107

4-10 Comparison of the SER for System Mode 1 in the Time-Invariant Waveguide for different $\gamma$ in the CNLLS Channel Estimator . . . . 108

4-11 Comparison of the SER for System Mode 1 in the Time-Invariant Waveguide for different choices of $a$ in the LMS Channel Estimator . 108

4-12 Depth Profile of Pressure Magnitude for All Channel Estimators after 100 Iterations . . . . . . . . . . . . . . . . . . . 109

4-13 Transient Response of Channel Estimators for Abrupt Change in Channel . . . . . . . . . . . . . . . . . . . . . . . . 112

4-14 Transient Response of Kalman Filter Channel Estimator for Gradual Change in the Waveguide . . . . . . . . . . . . . . . . . 113

4-15 Transient Response of CNLLS Channel Estimator for Gradual Change in the Waveguide . . . . . . . . . . . . . . . . . . . . . . 114

4-16 Transient Response of LMS Channel Estimator for Gradual Change in the Waveguide . . . . . . . . . . . . . . . . . . . . . . 114 


\section{Chapter 1}

\section{Introduction}

The shallow water channel is a waveguide for pressure waves, supporting propagation for a discrete set of normal modes in the far field, [1], [2]. Ocean acoustics problems such as internal wave tomography [3], [4] remote sensing [5], and noise propagation [6] [7] often discuss propagation in terms of modes. Numerous experiments have verified the modal model of propagation in the shallow water [8], [9], [2], [10], [11], but no mid-frequency (circa $400 \mathrm{~Hz}$ ) ocean experiment has successfully excited a single mode in a shallow water environment.

This thesis proposes an algorithm for controlling a vertical array of narrowband sources such that the pressure field in the shallow water acoustic channel measured at a reference location consists of only a single mode. While none of the individual aspects of the proposed algorithm are original, the synthesis of these elements produces a novel approach to exciting a single mode. The algorithm uses feedback control to obtain the desired pressure field at the reference hydrophone array. This technique is commonly used for pressure field control in open-air acoustics applications such as active noise control [12], [13] but is not often used in the underwater acoustics community. The specific feedback technique used to control the pressure field at the feedback array is the method of indirect control as described by Narendra and Annaswamy [14]. The control algorithm estimates the Green's function between 
each element of the source array and each hydrophone of the reference array. This matrix of estimated Green's functions is then inverted to determine the shading to apply to the source array in order to obtain the desired pressure field at the feedback hydrophone array. Both the acoustic model and control algorithm are simple and standard techniques in their respective fields. Although much more sophisticated approaches to the feedback control algorithm and ocean acoustics are possible, this simple approach will allow a clearer understanding of the problem in this preliminary investigation. If the algorithm proposed using these straightforward ideas proves successful here, further investigations can determine if more sophisticated approaches to both the acoustics and control can provide further gains.

Although the feedback control algorithm requires measurements of the sound speed profile at the feedback array, it obviates the need for detailed a priori information throughout the control volume in order to excite the desired pressure field. Previous work exciting a single mode in a laboratory waveguide exploited detailed environmental knowledge and time-invariance to find the array weights for exciting a single mode analytically [15], [16]. While these experiments successfully controlled the pressure in laboratory tanks in open loop mode, this approach appears unlikely to work in an arbitrary ocean situation without detailed knowledge of the environment. Range inhomogeneities in the environment may couple energy among modes or from the continuum into propagating modes before the pressure wave reaches the desired observation volume.

The distribution of propagating modes can be inferred from the pressure field sampled at the locations of the hydrophones in the reference array. This estimation problem is known as mode filtering in the ocean acoustics literature [17], [9]. A variety of algorithms have been proposed for solving this inverse problem. The sensitivity of this estimate to noise depends on the geometry of the hydrophone array and the mode filtering algorithm. A crucial issue for this thesis is determining how the error between the desired and observed pressure profiles is related to the error 
between the desired and actual mode distribution propagating in the channel. This problem and mode filtering are intrinsically related. The control theory framework developed in this thesis for determining the observability of modes can offer insight into this problem.

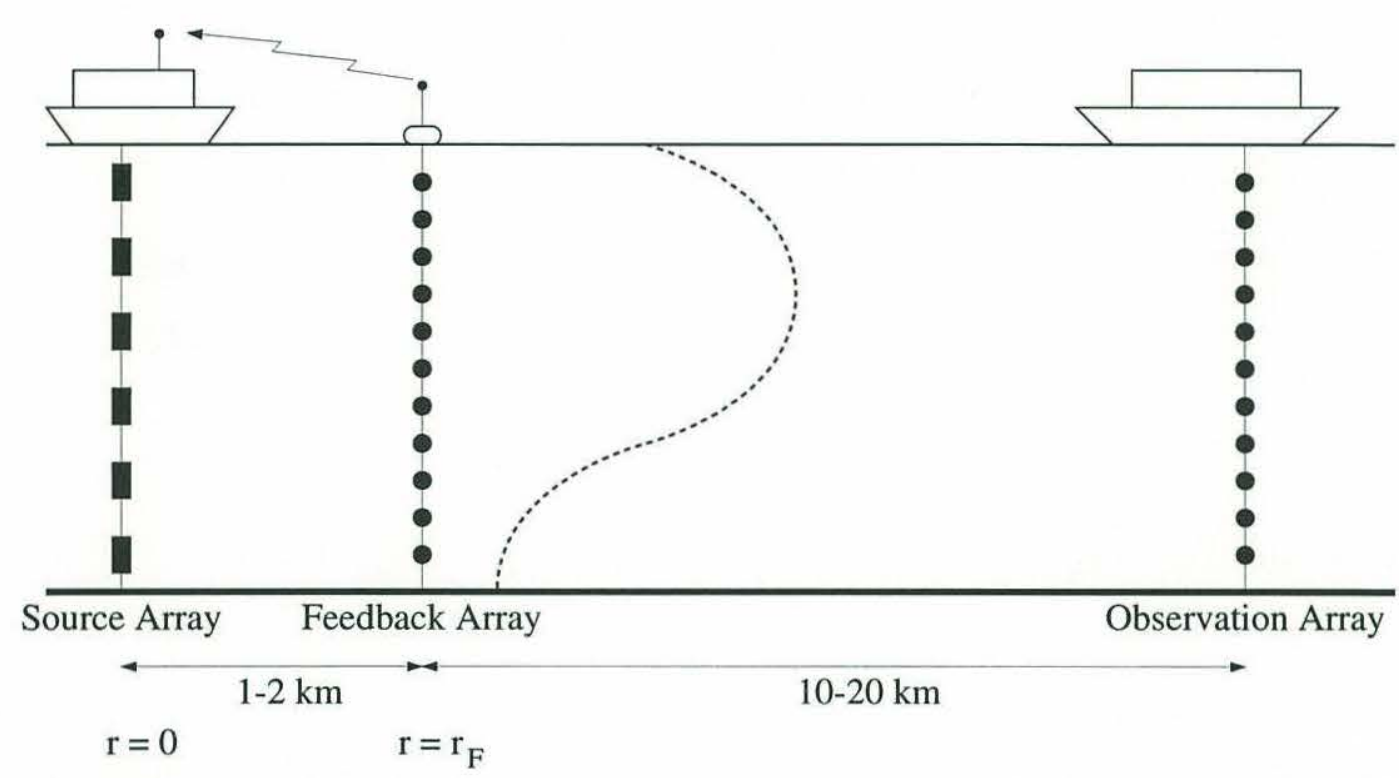

Figure 1-1: Proposed Experimental Setup

Figure 1-1 shows the proposed experiment configuration. The region between the source and feedback arrays will be called the feedback volume, while the region between the feedback and observation arrays will be referred to as the observation volume. The feedback algorithm attempts to control the pressure field such that only a single mode is propagating at the start of the observation volume, i.e., at the feedback array. This allows the observers at the observation array to be confident the pressure field they measure was generated by a high-fidelity mode impinging on the observation volume. The source array is tended by a ship, while the feedback array is located at $r_{F}=1-2 \mathrm{~km}$ downrange, at the start of the far field [18]. The far-field is defined as beginning at the range where all the significant energy propagating in the waveguide is described by the trapped modes. The feedback array transmits the pressure observed at its hydrophones back to the source ship over a ra- 
dio telemetry link. The scientific observation array is deployed $10-20 \mathrm{~km}$ downrange to measure the distribution of modes emerging from the observation region. For the purposes of the pressure received at the observation array, the feedback array can be considered a virtual source exciting the ocean at that location with a single mode. Knowing the pressure field entering the observation volume consisted of only a single mode, the modes arriving at the observation array can give information about the oceanographic properties of the intervening water mass.

Several studies have examined the effect of internal waves on acoustic propagation in the coastal environment. Lee [19] used a ray approach to demonstrate the presence of internal waves supported on a thermocline intensified the contrast in the shadow zones compared with a similar environment without the internal waves. Studies by Zhou, Zhang and Rogers [4] and Peregrym [3] simulated the propagation of acoustic normal modes through internal wave packets with sinusoidal displacement profiles. These simulations demonstrated a narrowband resonance coupling energy between modes when the difference between the modes' horizontal wavenumbers equaled the spatial wavenumber of the internal wave packet. More recent work by Preisig and Duda [20] have found the earlier studies may have exaggerated the narrowness of the resonance by using an unrealistically periodic displacement profile. All of this work indicates that given the pressure field entering the observation volume contains only one mode, the modes observed at the observation array contain significant information about the properties of any internal waves in that volume.

The problem of exciting a single mode using feedback control incorporates elements of underwater acoustics, mode filtering, control theory, and signal processing. The subsequent sections of this chapter summarize relevant aspects of each of these disciplines.

Chapter 2 describes the proposed control algorithm obtained by synthesizing the material covered in Chapter 1. First, a model is proposed for the acoustic channel of the feedback volume. The indirect control algorithm developed in this chapter 
first estimates the parameters of the channel model, then uses this model to choose the complex source weights to excite the desired pressure field at the feedback array. Several different estimators are presented for the identification of the channel model.

The proposed control algorithm is evaluated in Chapter 3 in simulations of a variety of shallow water ocean environments. All of the environments modeled are based on measured profiles from the South Continental Shelf off Martha's Vineyard. The propagation for these environments is simulated using the finite-element parabolic equation (FEPE) approximation to the wave equation [21].

In addition to the simulations, the thesis presents results from a series of experiments using the algorithm to control the pressure field at a hydrophone array in a scale model laboratory waveguide. These experiments, described in Chapter 4 , demonstrate the algorithm is robust enough to work in real time with actual sources, hydrophones, and acoustic waves propagating through water. The different algorithms for estimating the channel model are examined for both steady state and transient performance.

Chapter 5 makes conclusions about the algorithm proposed based on the simulations and experiments described in the thesis. In addition, this chapter suggests future directions for developing algorithms for single mode excitation and other possible uses for feedback control and single mode excitation in ocean acoustics.

\subsection{Normal Mode Model for Acoustic Propaga- tion}

The pressure field for a time-harmonic point source located at $\mathbf{r}_{0}$ in a range-independent environment can be described by the wave equation [22],

$$
\rho(z) \nabla \cdot\left[\frac{1}{\rho(z)} \nabla p(\mathbf{r})\right]+k^{2}(z) p(\mathbf{r})=-4 \pi \delta\left(\mathbf{r}-\mathbf{r}_{0}\right)
$$


where $\mathbf{r}=(r, \theta, z)$ is the observer's location in cylindrical coordinates, $\mathbf{r}_{0}=\left(0,0, z_{0}\right)$ is the source location, and the wavenumber $k(z)=\omega / c(z)$ is the ratio of the angular frequency to the depth-dependent sound speed. Assuming cylindrically symmetric solutions separable in range and depth of the form

$$
p(r, z)=\Psi_{m}(z) R_{m}(r)
$$

yields the normal mode solution for the field [22], [23], and [1]. Substituting Eq. 1.2 into the homogeneous form of Eq. 1.1 and separating the functions of depth and range gives the differential equations specifying the eigenfunctions for each of these coordinates:

$$
\begin{aligned}
\frac{1}{\rho(z)} \frac{d^{2} \Psi_{m}(z)}{d z^{2}}+\frac{d}{d z}\left[\frac{1}{\rho(z)}\right] \frac{d \Psi_{m}(z)}{d z}+\frac{k_{z m}^{2}(z)}{\rho(z)} \Psi_{m}=0 \\
\frac{1}{r} \frac{d}{d r}\left[r \frac{d R_{m}(r)}{d r}\right]+k_{m}^{2} R_{m}(r)=0
\end{aligned}
$$

where the separation constant is $k_{m}^{2}$, its square root $k_{m}$ is the horizontal wavenumber, and $k_{z m}(z)=\sqrt{k^{2}(z)-k_{m}^{2}}$ is the vertical wavenumber.

The total solution for the pressure will be a superposition of all solutions of the form of Eq. 1.2

$$
p(r, z)=\sum_{m} a_{m}\left(z_{0}\right) \Psi_{m}(z) R_{m}(r) .
$$

Substituting this expression into Eq. 1.1 and simplifying the resulting equation with Eq. 1.3 reveals

$$
\begin{aligned}
& R_{m}(r)=i \pi H_{0}^{(1)}\left(k_{m} r\right) \\
& a_{m}\left(z_{0}\right)=\Psi_{m}^{*}\left(z_{0}\right) / \rho\left(z_{0}\right),
\end{aligned}
$$

where $H_{0}^{(1)}\left(k_{m} r\right)$ is the zeroth-order Hankel function of the first kind, and the operator $(\cdot)^{*}$ denotes complex conjugation. Combining these gives the solution for the 
pressure field:

$$
p(r, z)=\frac{i \pi}{\rho\left(z_{0}\right)} \sum_{m} \Psi_{m}^{*}\left(z_{0}\right) \Psi_{m}(z) H_{0}^{(1)}\left(k_{m} r\right) .
$$

For the far field $\left(k_{m} r \gg 1\right)$, the Hankel function can be replaced by its asymptotic approximation to get

$$
p(r, z) \approx \frac{\sqrt{2 \pi} e^{i \pi / 4}}{\rho\left(z_{0}\right)} \sum_{m} \Psi_{m}^{*}\left(z_{0}\right) \Psi_{m}(z) \frac{e^{i k_{m} r}}{\sqrt{k_{m} r}} .
$$

In general, the sound speed profile $c$ and density $\rho$ are functions of range as well as depth. Consequently, the solutions to Eq. 1.3 vary with range. The solutions to the depth eigenfunction equation for the hydrographic and boundary conditions at a given range are known as the local modes at that range. The pressure field at range $r$ can be described as a superposition of the local modes $\Psi_{m}(z ; r)$, or

$$
p(r, z)=\sum_{m} d_{m}\left(r, z_{0}\right) \Psi_{m}(z ; r),
$$

where $d_{m}\left(r, z_{0}\right)$ are the complex mode coefficients. If the channel is linear, the pressure field excited by an array of sources can be written as a superposition of solutions of the form of Eq. 1.10. Equivalently, the complex mode coefficients can be considered to be functions of the source depth vector $\mathbf{z}_{s}$ and the complex source weights $\mathbf{w}$, in addition to $r$. The pressure field generated by a vertical array of sources at depths $\mathbf{z}_{s}$ weighted by $\mathbf{w}$ is

$$
p(r, z)=\sum_{m} d_{m}\left(r, \mathbf{z}_{s}, \mathbf{w}\right) \Psi_{m}(z ; r)
$$

The proposed algorithm attempts to choose $\mathrm{w}$ such that only one mode coefficient $d_{m_{0}}$ is non-zero at the feedback hydrophone array at $r=r_{F}$.

Acoustic propagation of range-dependent modes is categorized as adiabatic or coupled. The adiabatic model of propagation assumes no energy is exchanged between different modes although the local modes $\Psi_{m}(z ; r)$ may vary slowly with range. 
In coupled propagation, energy is exchanged between the local modes due to range variations in bathymetry and sound speed [24],[25].

The normal mode formulation for acoustic propagation is especially attractive in shallow water environments at medium to low frequency. In this scenario, the horizontal wavenumber spectrum can be segmented into three classes of waves. The propagating, or "trapped," modes are the solutions to the eigenfunction equations (Eqs. 1.3 and 1.4) such that the horizontal wavenumber $k_{m}$ is real, or at least has only a very small imaginary part due to medium attenuation during propagation. The evanescent modes have vertical profiles which are solutions to the depth eigenfunction equation (Eq. 1.3), but with $k_{z m}>k$, so $k_{m}=\sqrt{k^{2}-k_{z m}^{2}}$ is imaginary. These modes decay exponentially in range. In an ideal waveguide with perfectly reflecting boundaries, the trapped and evanescent modes contain all the energy. For a realistic ocean waveguide, the situation is complicated by the absence of a perfectly reflecting bottom. The solutions in this environment can be found by taking the limit as the ideal boundary goes to infinite depth [22]. This results in a continuum of horizontal wavenumbers from the solutions to Eqs. 1.3 and 1.4. A discrete finite subset of these solutions propagate in range with little or no attenuation; these solutions are the trapped modes for the waveguide. The rest of the solutions constitute the modal continuum. The contribution of the continuum is significant at short ranges, but quickly decays in range to leave only the trapped modes in the far field.

Many shallow water environments have bathymetry that is sufficiently rangevarying to invalidate the adiabatic propagation model. Desaubies [25] quantified measures indicating the importance of modal coupling in an environment. He found that the precise conditions when the adiabatic approximation breaks down depends on the quantity of interest, i.e., pressure, transmission loss, propagation time, etc.... For many of the quantities of interest, Desaubies found the conditions when the adiabatic approximation became invalid depended in subtle and sometimes intricate ways on the rate of change of the environment in range. Because the coupling induced 
by the bottom or range-inhomogeneities in the water column can be strong, it is very difficult to compute the source weights $\mathbf{w}$ to excite only a single mode without very extensive and accurate environmental measurements.

Many researchers have investigated and validated the modal model of shallow water propagation. The seminal work in shallow water modal propagation is the monograph "Theory of Propagation of Explosive Sound in Shallow Water" by C. L. Pekeris [2]. This paper develops the so-called "Pekeris waveguide," consisting of an isovelocity water layer, bounded above by a pressure release boundary and below by a higher-velocity isovelocity halfspace. Using this model and the theory of normal modes, the paper predicts the features of the pressure pulse caused by an impulsive source (TNT detonation) on the ocean bottom [26].

Bucker did significant work verifying the modal model of propagation for the shallow water channel. In [10], he compared experimental and theoretical mode curves for explosive sources in the Bering Sea, obtaining close agreement. The timefrequency distribution of the arrivals allowed him to identify the various modes in the energy observed at a single hydrophone. In a later paper [27], he computed expressions for the normal mode shapes for a variety of analytic sound speed profiles, as well as one measured experimental environment. Bucker used the computed mode shapes to predict transmission loss as a function of range in that experiment, and found reasonable agreement with the observed values.

Ferris et al. [11], [8], transmitted time-windowed sinusoidal pulses from a single $400 \mathrm{~Hz}$ source to a nine element vertical array of hydrophones $10 \mathrm{~km}$ downrange. The differing group delays of the modes resulted in different travel times between the source and receiver. These travel times differed by enough that two, and sometimes three, distinct modes could be temporally resolved at the receiver without any spatial processing. The vertical profiles of these modes across the array corresponded well with the computed mode shapes for the sound speed profile. The later paper, [8], also employed a simple spatial filter which projected the pressure field against samples 
of the theoretically derived mode shapes to verify the mode strength as a function of source depth matched the computed mode shapes.

Tindle et al. [9] performed an experiment very clearly demonstrating shallow water propagation was well-modeled by modes. They found a site almost perfectly homogeneous in range and depth. Over a range of $5 \mathrm{~km}$, the bottom depth was $50 \pm 1 \mathrm{~m}$, with a sound speed profile that was nearly perfectly homogeneous in depth and range with value $1508.7 \pm 0.3 \mathrm{~m} / \mathrm{s}$. The analytically derived depth eigenfunctions for an isovelocity water column fit this experimental environment very well. By running experiments transmitting windowed sinusoids centered at 60, 100, and 140 $\mathrm{Hz}$ and using the least squares mode filter which is discussed in Section 1.3, they were able to resolve the predicted arrivals of one, two and three modes, respectively.

Buckingham [28], [29] analytically derived an approximate expression for the modes for a downslope wedge environment with an isovelocity water column over an isovelocity halfspace bottom. Although the propagation for this environment can be approximated with the coupled mode model, he demonstrated the true eigenfunctions of the wave equation for this geometry were circularly curved wavefronts, centered on the apex of the wedge, sinusoidally varying in angle. These wedge modes propagate downslope without coupling energy among them, and thus are the true modes of the system. Since the wedge possesses spherical symmetry rather cylindrical, it is intuitively reasonable that the azimuthal angle plays the role depth played in cylindrical geometry with a flat bottom.

Tindle, Hobaek, and Muir [30], [31] confirmed Buckingham's theory in a series of thorough and elegant experiments investigating modal propagation in a scale model downslope environment. Their laboratory tank was $93 \mathrm{~cm}$ wide and $10 \mathrm{~m}$ long with $10 \mathrm{~cm}$ of water at the source over a $20 \mathrm{~cm}$ deep sand bottom. The bottom could be tilted at any angle between $0^{\circ}$ and $9^{\circ}$. Their source transmitted pulses centered on $80 \mathrm{kHz}$ and the pressure field in the tank was measured by a hydrophone moved between successive transmissions to create a synthetic discrete array. Tindle et al. 
observed the signals propagated downslope with curved wavefronts centered on the apex of the wedge as predicted by Buckingham. They resolved these modes by spatially filtering the pressure field using the least-squares technique of Tindle et al. in [9]. Comparing the results obtained using a mode filter designed with the local vertical modes to those obtained when the filter used the curved wedge modes demonstrated that only the latter propagated without coupling.

Several experiments examined single mode excitation in laboratory tanks. Clay and Huang attempted to transmit and receive a single mode in a laboratory tank as part of an experiment to measure backscattering from fish [15]. The tank they used had $25 \mathrm{~cm}$ of water and they transmitted at a frequency of $220 \mathrm{~Hz}$. The experimental waveguide had pressure-release surfaces both above and below, thus supported roughly 70 modes at this frequency. Clay and Huang attempted to excite only the first mode using 8 source transducers, shading these sources with samples of the desired mode shape quantized to three levels. The same array and shading were used as a receiver. Under these conditions of gross undersampling and coarse quantization, it is not surprising that the received pressure field roughly matched the shading. While Clay and Huang claim this indicates only the first mode was excited, it also seems possible they synthesized an approximation to this crudely quantized first mode shape using all the modes as basis functions. Thus, the channel may not have contained just one mode, but may actually have contained many propagating modes whose superposition approximated a quantized version of the first mode shape. Because Clay and Huang's receiver undersampled the channel spatially and they gave no information about temporal dispersion, it is not possible to conclude with certainty from their results what modes were propagating in the channel. Also, their experiment used fixed array weights built into the source/receiver array. The laboratory environment was almost certainly time-invariant, so the fixed nature of the weights was probably not an issue. Because it could not respond to any temporal variations in the channel, this open-loop control algorithm probably would not 
perform well in many ocean environments.

One of the best experiments for single-mode generation in a laboratory setting was performed by Gazanhes and Garnier, [16]. They used a tank with only $57 \mathrm{~mm}$ of water over a sand bottom. At their working frequency of $124 \mathrm{kHz}$, this environment only supported 5 trapped modes, making control and estimation of the modes much more tractable than the Clay and Huang experiment. Using the Pekeris model of propagation, Gazanhes and Garnier computed the mode shapes for the channel and used samples of these shapes to weight 15 independent piezoelectric transducers. Although this is not the optimal weighting in the least-squares sense, it is a much better discrete approximation to the orthogonality condition than Clay and Huang's very coarse sampling and weighting. Gazanhes and Garnier confirmed that they excited only a single mode using synthetic aperture arrays in both range and depth. Although their control scheme is also open loop, it works well because they have a known time-invariant environment. This is encouraging evidence that it is possible to excite a single mode given an accurate channel estimate.

This concludes our review of previous work on mode propagation both in the ocean and in laboratory settings. The next section describes the Green's function for underwater acoustics. The Green's function is the foundation of our model for the channel between the source array and feedback array.

\subsection{Green's Function}

The Green's function characterizes the acoustic propagation between a source and receiver at a given frequency. To find this function in regions of constant density, simplify the Helmholtz equation (Eq. 1.1) to obtain

$$
\left[\nabla^{2}+k^{2}(\mathbf{r})\right] \mathbf{p}(\mathbf{r})=-4 \pi \delta\left(\mathbf{r}-\mathbf{r}_{\mathbf{0}}\right)
$$


for a point source at $\mathbf{r}_{0}$. The solution to this equation, $\mathbf{p}(\mathbf{r})=G\left(\mathbf{r}, \mathbf{r}_{0}\right)$, is the Green's function [22], [32]. In linear system theory terms, the Green's function is the transfer function between a point source at $\mathbf{r}_{0}$ and a receiver at $\mathbf{r}$ evaluated at a single frequency $\omega$. The Green's function is a very general model for propagation, and is still valid in many environments where a discrete set of normal modes cannot accurately model propagation. For a driving term $f\left(\mathbf{r}_{0}\right)$ it can be shown the pressure field is

$$
p(\mathbf{r})=\int_{V_{0}} G\left(\mathbf{r}, \mathbf{r}_{0}\right) f\left(\mathbf{r}_{\mathbf{0}}\right) d \mathbf{r}_{0}+\frac{1}{4 \pi} \int_{S_{0}}\left[G\left(\mathbf{r}, \mathbf{r}_{0}\right) \frac{\partial p\left(\mathbf{r}_{\mathbf{0}}\right)}{\partial n_{0}}-p\left(\mathbf{r}_{\mathbf{0}}\right) \frac{\partial G\left(\mathbf{r}, \mathbf{r}_{0}\right)}{\partial n_{0}}\right] d \mathbf{r}_{0}
$$

The first integral incorporates the contribution of all sources in the volume in question $V_{0}$, while the second integral includes the effect of the boundary conditions on $S_{0}$, the surface surrounding $V_{0}$.

If the acoustic channel between source and receiver arrays is a linear system with respect to the complex weight vector of the source array, the contribution of the second integral in Eq. 1.13 must be zero, and there must not be any other significant sources in the volume. The condition that the second integral of Eq. 1.13 be zero is the spatial analog of the initial rest boundary condition for a time-domain linear system. In physical terms, this corresponds to an absence of sources outside the volume in question, $V_{0}$. In general, the ocean may be considered time-invariant for the relatively brief propagation times between the feedback and source arrays. Under these conditions, the channel is well-modeled by a linear, time-invariant (LTI) system. Complex exponentials are eigenfunctions of LTI systems, so the Green's function completely characterizes the behavior of the channel at the frequency of excitation. Because it is a very general model for propagation, the Green's function can incorporate effects from any spatial frequencies in the modal continuum which are not one of the trapped modes, but still which couple energy back into the propagating modes, in addition to summarizing any coupling of energy among the propagating modes. 
In environments which are completely represented by a discrete set of normal modes, the depth-dependent Green's function has poles at the horizontal wavenumbers of the modes, indicating the pressure field consists predominantly of energy propagating at those spatial frequencies. Because the set of environments where the Green's function accurately models propagation is a superset of those accurately modeled by either the adiabatic or coupled mode models, it is a good model for the control algorithm to use to summarize the acoustic propagation through the feedback volume. The model allows single mode excitation in any environment well-modeled by either adiabatic or coupled mode propagation, and possibly in some more complex environments as well.

\subsection{Mode Filters}

The problem of estimating the coefficients of the modes propagating at a given location from samples of the pressure field obtained at hydrophones, known as mode filtering, is a common one in ocean acoustics. The feedback control algorithm must determine how the error between the desired and observed pressure profile at the feedback array is related to the error between the desired and excited mode coefficients. These two problems are closely related, and in addition to reviewing common algorithms used for mode filtering, this section will also examine the similarity between these problems.

The pressure field generated by $M$ propagating modes spatially sampled by a vertical array of $N$ hydrophones can be written as

$$
\left[\begin{array}{c}
p\left(z_{1}\right) \\
\vdots \\
p\left(z_{N}\right)
\end{array}\right]=\left[\begin{array}{ccc}
\Psi_{1}\left(z_{1}\right) & \ldots & \Psi_{M}\left(z_{1}\right) \\
\vdots & \ddots & \vdots \\
\Psi_{1}\left(z_{N}\right) & \ldots & \Psi_{M}\left(z_{N}\right)
\end{array}\right]\left[\begin{array}{c}
d_{1} \\
\vdots \\
d_{M}
\end{array}\right]+\left[\begin{array}{c}
n\left(z_{1}\right) \\
\vdots \\
n\left(z_{N}\right)
\end{array}\right]
$$


or in vector notation

$$
\mathrm{p}=\Psi \mathrm{d}+\mathrm{n}
$$

where $\mathbf{n}$ is the vector of observation noise at the hydrophone locations.

Linear mode filters estimate the mode coefficients as a linear function of the observed pressure samples. For Sections 1.3.1 through 1.3.3, the mode coefficient vector will be considered a deterministic but unknown quantity to be estimated. In This linear function can be represented by a matrix $\mathbf{H}$ multiplying the observed pressure field $\mathbf{p}$, i.e.,

$$
\hat{\mathrm{d}}=\mathbf{H p}=\mathbf{H} \Psi \mathrm{d}+\mathrm{Hn} .
$$

The various mode filters discussed in this section correspond to different choices for $\mathbf{H}$ proposed in the literature of generalized inverses [33], [34] and stochastic estimation [35]. For mode filters of this form, the covariance of the mode coefficient vector estimate $K_{\hat{\mathrm{d}} \hat{\mathbf{d}}}=E\left\{\hat{\mathbf{d}} \hat{\mathbf{d}}^{\mathrm{H}}\right\}-E\{\hat{\mathbf{d}}\} E\left\{\hat{\mathbf{d}}^{\mathrm{H}}\right\}$ is

$$
K_{\hat{\mathrm{d}} \hat{\mathrm{d}}}=\mathrm{H} K_{\mathrm{nn}} \mathrm{H}^{\mathrm{H}},
$$

where $K_{\mathbf{n n}}$ is the spatial covariance of the noise vector $\mathbf{n}$, and $(\cdot)^{\mathrm{H}}$ denotes the Hermitian, or conjugate-transpose, operator. Thus, the covariance of the estimated mode coefficients depends entirely on the noise process covariance $\mathbf{K}_{\mathbf{n n}}$ and the mode filter $\mathbf{H}$. The remainder of this section reviews the common choices for $\mathbf{H}$ in the ocean acoustics literature.

\subsubsection{Sampled Mode Shapes Mode Filter}

A common choice for $\mathbf{H}$ in mode propagation experiments is $\Psi^{\mathrm{H}}$. The motivation for this choice is that the mode shapes are orthonormal when integrated as a function of continuous depth and weighted by the density profile [22]. However, spatially sampling the modes does not necessarily preserve the orthogonality of the modes, 
i.e., $\Psi^{\mathrm{H}} \Psi \neq \mathrm{I}$. This lack of orthogonality can introduce a bias into the mode estimate. Assuming the noise vector $\mathbf{n}$ is zero-mean, the expected value of the mode coefficient estimator is

$$
E\{\hat{\mathbf{d}}\}=\Psi^{\mathrm{H}} \Psi \mathrm{d},
$$

giving a bias of $\left(I-\Psi^{\mathrm{H}} \Psi\right)$ d. Theoretically, as more hydrophones are added to sample the water column more finely in depth, the sampled modes matrix $\Psi$ becomes arbitrarily close to orthogonal, making the effect of the bias negligible. Realistically, it is impractical to deploy a vertical array of hydrophones spanning the entire sediment layer. Thus, even in the limiting case of a continuous array of hydrophones spanning the water column, some bias will still be present due to the unsampled pressure field in the bottom [36]. Many experiments including Ferris [8], and Clay and Huang [15] used the sampled mode shape mode filter, assuming the samples of the orthogonal mode functions are themselves orthogonal without examining the potential bias of the sampled mode shape filter. Tindle, Hobaek, and Muir [31] and Gazanhes and Garnier [16] used the same mode filter but examined the "cross-talk" introduced by sampling to verify the bias was negligible for the purposes of their experiments.

The mode coefficients may be difficult to determine from the pressure samples taken at the hydrophone locations if the hydrophone array either has fewer hydrophones than the number of propagating modes, or if the hydrophones do not fully span the water depth. The first difficulty will be referred to as undersampling, while the second will be referred to as poorly-conditioned sampling. The singular value decomposition (SVD) [37], an orthogonal matrix factorization, provides insight on how these problems affect the mode estimator. The SVD factors $\Psi$ as $\mathrm{U}_{\Psi} \boldsymbol{\Sigma}_{\Psi} \mathrm{V}_{\Psi}{ }^{\mathrm{H}}$, where $\mathrm{U}_{\Psi}$ and $\mathrm{V}_{\Psi}$ are orthonormal matrices. Assuming the number 
of hydrophones exceeds the number of trapped modes, i.e., $N>M$,

$$
\boldsymbol{\Sigma}=\left[\begin{array}{cccc}
\sigma_{1} & 0 & \ldots & 0 \\
0 & \ddots & \ddots & \vdots \\
\vdots & \ddots & \ddots & 0 \\
0 & \ldots & 0 & \sigma_{M} \\
\hline & 0 & &
\end{array}\right]
$$

where $\sigma_{\Psi 1} \geq \sigma_{\Psi 2} \geq \ldots \geq \sigma_{\Psi M} \geq 0$ are called the singular values of $\Psi$. In the undersampled case, with $N<M$, the mode filtering problem is underdetermined and $\hat{\mathbf{d}}$ cannot be uniquely determined from $\mathbf{p}$. Practically, this is usually not a problem for mid-to-low frequencies in the shallow water environment, since it is not difficult or expensive to construct an array with more hydrophones than the environment has propagating modes. Even if $N \geq M$, the choice of hydrophone locations may give a poorly-conditioned problem for determining $\hat{\mathrm{d}}$. Although the problem is now nominally over-determined, poorly-conditioned sampling will cause $\Psi$ to become rank-deficient or nearly rank-deficient. This will make it impossible to estimate $\hat{\mathbf{d}}$ reliably in the presence of noise. Golub and Van Loan [37] demonstrate poorlyconditioned sampling results in one or more of the singular values $\sigma_{m}$ becoming very small or zero. Rewriting the bias $B(\mathrm{~d})$ as

$$
\begin{aligned}
& B(\mathrm{~d})=\left(I-\Psi^{\mathrm{H}} \Psi\right) \mathrm{d} \\
& =\mathrm{V}_{\Psi}\left(I-\left[\begin{array}{ccc}
\sigma_{1}^{2} & & 0 \\
& \ddots & \\
& & \sigma_{M}^{2}
\end{array}\right]\right) \mathrm{V}_{\Psi^{H}}{ }^{H} \mathrm{~d}
\end{aligned}
$$

makes it clear decreasing any of the $\sigma_{m}$ 's to zero will increase the bias of the estimator. 
The covariance of the sampled mode shapes estimator depends on the covariance of the noise vector $K_{\mathrm{nn}}$. Two common models for noise in the shallow water channel are spatially white noise $\left(K_{\mathrm{nn}}=\sigma_{n}^{2} \mathbf{I}\right)$ and the Kuperman-Ingenito surface noise model [6]. The Kuperman-Ingenito model proposes that when the noise is dominated by surface generated noise, i.e., noise due to wind, surface waves, etc..., it will have a spatial covariance of the form

$$
K_{\mathrm{nn}}=\Psi\left[\begin{array}{cccc}
\sigma_{\tilde{d}_{1}}^{2} & 0 & \cdots & 0 \\
0 & \sigma_{\tilde{d}_{2}}^{2} & \ddots & \vdots \\
\vdots & \ddots & \ddots & 0 \\
0 & \cdots & 0 & \sigma_{\tilde{d}_{M}}^{2}
\end{array}\right] \Psi^{\mathrm{H}}=\Psi K_{\tilde{\mathrm{d}} \tilde{\mathrm{d}}} \Psi^{\mathrm{H}}
$$

where $\sigma_{\tilde{d}_{1}}^{2}, \ldots, \sigma_{\tilde{d}_{M}}^{2}$ are functions of the mode profiles and surface noise processes. The vector $\tilde{\mathbf{d}}$ is the mode coefficients of the noise process at the array.

For the spatially white case, Eq. 1.17 becomes

$$
K_{\hat{\mathrm{d}} \hat{\mathrm{d}}}=\sigma_{n}^{2} \sum_{m=1}^{M} \sigma_{\Psi m}^{2} \mathbf{v}_{\boldsymbol{\Psi} m} \mathbf{v}_{\boldsymbol{\Psi} m}{ }^{\mathrm{H}},
$$

where $\mathbf{v}_{\boldsymbol{\Psi} m}$ are the columns of $\mathbf{V}_{\boldsymbol{\Psi}}$. The Kuperman-Ingenito model gives

$$
K_{\hat{\mathrm{d}} \hat{\mathrm{d}}}=\left(\sum_{m=1}^{M} \sigma_{\Psi m}^{2} \mathbf{v}_{\boldsymbol{\Psi} m} \mathbf{v}_{\boldsymbol{\Psi}}{ }^{\mathrm{H}}\right)\left[\begin{array}{cccc}
\sigma_{\tilde{d}_{1}}^{2} & 0 & \ldots & 0 \\
0 & \sigma_{\tilde{d}_{2}}^{2} & \ddots & \vdots \\
\vdots & \ddots & \ddots & 0 \\
0 & \ldots & 0 & \sigma_{\tilde{d}_{M}}^{2}
\end{array}\right]\left(\sum_{m=1}^{M} \sigma_{\Psi m}^{2} \mathbf{v}_{\boldsymbol{\Psi} m} \mathbf{v}_{\Psi_{m}}{ }^{\mathrm{H}}\right)
$$

Poorly-conditioned sampling decreases some values of $\sigma_{\Psi m}$, but its effect on the estimator covariance does not grow more extreme as the conditioning of $\Psi$ becomes 
more severe. Once a particular singular value grows negligible compared to other singular values, further decreases in its value are insignificant in the covariance computations in Eqs. 1.22 and 1.23. At the other extreme, when the channel is highly oversampled, i.e., $N \rightarrow \infty$, the $\sigma_{\Psi m}$ 's approach 1, so

$$
\sum_{m=1}^{M} \sigma_{\Psi m}^{2} \mathbf{v}_{\boldsymbol{\Psi} m} \mathbf{v}_{\boldsymbol{\Psi} m}{ }^{\mathrm{H}} \rightarrow \mathbf{V}_{\boldsymbol{\Psi}} \mathbf{V}_{\boldsymbol{\Psi}}{ }^{\mathrm{H}}=\mathbf{I}
$$

and Eqs. 1.22 and 1.23 simplify to $K_{\hat{\mathrm{d}} \hat{\mathrm{d}}}=\sigma_{n}^{2} I$ and $K_{\hat{\mathrm{d}} \mathrm{d}}=K_{\tilde{\mathrm{d}} \tilde{\mathrm{d}}}$, respectively.

Thus, poorly-conditioned sampling of the water column by the hydrophone array will increase the bias of the sampled mode shape filter, but will not effect the estimator covariance significantly. Oversampling the channel so that the inner product of the sampled mode shapes closely approximates the integral of the continuous mode shapes can reduce the bias to leave only a small contribution from the unsampled bottom, and result in an estimator covariance reflecting the underlying nature of the noise process.

\subsubsection{Pseudo-Inverse Mode Filter}

The pseudo-inverse mode filter results from choosing $\hat{\mathrm{d}}$ to minimize the squared error between $\Psi \hat{\mathbf{d}}$ and $\mathbf{p}$. Tindle et al. [9] appears to be the first reference in the ocean acoustics literature to formulate the mode estimation problem in this least squares sense. The resulting mode filter $\mathbf{H}=\left(\Psi^{\mathrm{H}} \Psi\right)^{-1} \Psi^{\mathrm{H}}$, denoted $\Psi^{\dagger}$, is called the pseudo-inverse or Penrose-Moore inverse of $\Psi$ [37], [33]. This name results from the fact $\Psi^{\dagger} \Psi=I$. A consequence of this property is that if $E\{\mathrm{n}\}=0$, the mode estimate obtained using $\mathbf{H}=\Psi^{\dagger}$ is unbiased. However, if the hydrophone array gives a poorly-conditioned sampling of the modes, this estimator becomes very sensitive to noise. 
For the spatially white noise model, Eq. 1.17 gives

$$
\begin{aligned}
K_{\hat{\mathbf{d}} \hat{\mathbf{d}}} & =\sigma_{n}^{2} \mathbf{V}_{\Psi} \Sigma_{\Psi}^{\dagger}\left(\Sigma_{\Psi}^{\dagger}\right){ }^{\mathrm{H}} \mathbf{V}_{\Psi}{ }^{\mathrm{H}} \\
& =\sigma_{n}^{2} \sum_{m=1}^{M} \sigma_{\Psi m}^{-2} \mathbf{V}_{\Psi m} \mathbf{V}_{\Psi m}{ }^{\mathrm{H}} .
\end{aligned}
$$

Although this estimate will remain unbiased so long as $N \geq M$, for very short aperture arrays the estimator will have a very large covariance as one more or more singular values $\sigma_{\Psi m}$ go to zero. When a full aperture array oversamples the channel, the singular values of $\Psi$ approach 1 , and Eq. 1.25 approaches $\sigma_{n}^{2} \mathbf{I}$.

Substituting the Kuperman-Ingenito noise model and the pseudo-inverse mode filter into Eq. 1.17 gives

$$
\begin{aligned}
K_{\hat{\mathrm{d}} \hat{\mathrm{d}}} & =\Psi^{\dagger} \Psi K_{\tilde{\mathrm{d} \tilde{d}}} \Psi^{\mathrm{H}} \Psi^{\dagger \mathrm{H}} \\
& =K_{\tilde{\mathrm{d}} \tilde{\mathrm{d}}}
\end{aligned}
$$

which is intuitively sensible, as $K_{\tilde{\mathrm{d}} \tilde{\mathrm{d}}}$ is the covariance of the noise process as it is coupled into the channel by the modes. Ideally, this covariance is unchanged by reductions in the array aperture. Practically, the mode filter $\Psi^{\dagger}$ is usually based on an estimate of $\Psi$ computed by numerical integration of an observed or estimated sound speed profile. As the $\sigma_{m}$ 's of the true $\Psi$ grow smaller as the array aperture decreases and the sampling becomes poorly-conditioned, the bias and covariance of the pseudo-inverse mode filter can become very sensitive to errors between the $\Psi$ obtained by numerical integration and the actual $\Psi$ of the ocean channel.

For the spatially white Gaussian noise case, the pseudo-inverse mode filter can be shown to be the maximum-likelihood (ML) estimator. In general, it is not an efficient estimator and does not attain the Cramer-Rao Bound (CRB) for the variance of unbiased estimators unless all the singular values $\sigma_{\Psi m}$ are equal. Poorly-conditioned sampling of the channel can push the covariance of this mode filter arbitrarily far 
from the CRB as some of the singular values grow very small. If an efficient estimator exists, it is the ML estimator [35]; since the ML estimator is not efficient, no efficient estimator exists for the spatially white noise model.

The pseudo-inverse mode filter is also the ML estimator for the KupermanIngenito noise model. For this case, this conclusion is not very informative since $\mathbf{n}$ is defined to be in the range of $\Psi$ from the formulation of the noise model. The existence of the ML estimator depends on the Kuperman-Ingenito model perfectly describing the noise process, since if $\mathbf{n}$ contains any component in the orthogonal complement to the range of $\Psi$, the conditional probability density $p_{\mathbf{P} \mid \mathbf{D}}(\mathbf{p} \mid \mathbf{d})=0$ [38], and the ML estimate is meaningless since no set of mode coefficients $\mathbf{d}$ could have produced the observed signal. For this noise model, the Fisher Information Matrix can be shown to be $K_{\tilde{\mathrm{d}} \tilde{\mathrm{d}}}^{-1}$, so the CRB for the mode estimator is $K_{\tilde{\mathrm{d}} \tilde{\mathrm{d}}}$, the variance of the underlying noise process in each mode. The pseudo-inverse mode filter attains this bound on the variance, and is an efficient estimator.

\subsubsection{Diagonal Weighting}

As noted above, when the array has poorly-conditioned sampling of the modes, one or more of the singular values of $\Psi$ will grow very small. This causes $\Psi^{\mathrm{H}} \Psi$ to be singular or nearly singular, and the computation of the inverse of this matrix can grow numerically sensitive. One method of compensating for this is to modify the error function being minimized to include a term proportional to the magnitude squared of the estimated mode coefficient vector $\hat{\mathrm{d}}$ [39]. The quantity to be minimized is then

$$
e=\|\mathbf{p}-\Psi \hat{\mathbf{d}}\|^{2}+\beta\|\hat{\mathbf{d}}\|^{2}
$$


where $\beta$ is a scale factor indicative of the relative importance of the two terms in the error expression. The estimator minimizing this quantity is

$$
\hat{\mathrm{d}}_{\mathrm{DW}}=\left(\Psi^{\mathrm{H}} \Psi+\beta \mathbf{I}\right)^{-1} \Psi^{\mathrm{H}} \mathbf{p}
$$

This expression is very similar to the pseudo-inverse, except for a small diagonal matrix $\beta \mathbf{I}$ has been added to $\Psi^{\mathrm{H}} \Psi$ before inversion to alleviate conditioning problems. The $\beta \mathbf{I}$ term is often referred to as the white noise sensitivity term. The addition of this term places a lower bound of $\beta$ on the singular values of $\left(\Psi^{\mathrm{H}} \Psi+\beta \mathbf{I}\right)$, and thus limits the covariance of the estimator shown in Eq. 1.25, since no $\sigma_{\Psi m}^{2}$ for the diagonally-weighted inverse can exceed $\beta^{-2}$. For this reason, this approach is often referred to as diagonal loading or weighting. While this estimator does not possess many of the nice theoretical properties of the pseudo-inverse mode filter, in many scenarios it is computationally more stable, especially at relatively high noise levels.

\subsubsection{Maximum A Posteriori Mode Filters}

The maximum a posteriori (MAP) mode filter chooses $\hat{\mathbf{d}}_{\mathrm{MAP}}$ to maximize the probability density function $p_{\mathbf{D} \mid \mathbf{P}}(\mathbf{d} \mid \mathbf{p})$ based on the observed pressure field, $\mathbf{p}$. This approach assumes the mode coefficient vector $\mathbf{d}$ is an unknown, random quantity to be determined. The mode filter also assumes knowledge of the probability density functions of both the stochastic processes generating the mode coefficients $d$ and the noise $\mathbf{n}$. For the case when both these processes are Gaussian, the MAP estimate is identical to the minimum mean-squared error estimate (MMSE) [35]. A very important feature of the MAP mode filter is that in many environments it gracefully transitions from the pseudo-inverse filter to the sampled mode shape filter as the conditioning of $\Psi$ deteriorates. Between these extremes, the performance of the MAP filter exceeds either of the other two filters, and the MAP filter never exhibits the poor performance shown by the pseudo-inverse or sampled mode shape filters when 
these filters are applied to a problem whose conditioning is inappropriate for their strengths. If the mode coefficients are well-modeled by a Gaussian process with zero mean and covariance $K_{\mathrm{dd}}$, while the noise is also Gaussian, zero-mean, uncorrelated with $\mathrm{d}$, and has covariance $K_{\mathrm{nn}}$, the MAP mode filter can be written as

$$
\hat{\mathrm{d}}_{\mathrm{MAP}}=K_{\mathrm{xx}} \Psi^{\mathrm{H}} K_{\mathrm{nn}}^{-1} \mathbf{p},
$$

where

$$
K_{\mathrm{xx}}^{-1}=K_{\mathrm{dd}}^{-1}+\Psi^{\mathrm{H}} K_{\mathrm{nn}}^{-1} \Psi .
$$

Some insight into the performance of this mode filter may be gained by considering the somewhat unrealistic case when the modes are independent and identically distributed, i.e., $K_{\mathrm{dd}}=\sigma_{d}^{2} \mathbf{I}$, and the noise is spatially white with $K_{\mathrm{nn}}=\sigma_{n}^{2} \mathbf{I}$. Assuming there are more hydrophones than modes $(M>N)$, Eq. 1.29 reduces to

$$
\hat{\mathbf{d}}_{\mathrm{MAP}}=\mathbf{V}_{\Psi}\left[\begin{array}{cccc}
\frac{\sigma_{d}^{2} \sigma_{\Psi 1}}{\sigma_{d}^{2} \sigma_{\Psi 1}^{2}+\sigma_{n}^{2}} & 0 & \cdots & 0 \\
0 & \frac{\sigma_{d}^{2} \sigma_{\Psi 2}}{\sigma_{d}^{2} \sigma_{\Psi 2}^{2}+\sigma_{n}^{2}} & \ddots & \vdots \\
\vdots & \ddots & \ddots & 0 \\
0 & \cdots & 0 & \frac{\sigma_{\sigma}^{2} \sigma_{\Psi M}}{\sigma_{d}^{2} \sigma_{\Psi M}^{2}+\sigma_{n}^{2}}
\end{array}\right] \mathbf{0}_{\Psi}^{\mathrm{H}} \mathbf{p}
$$

Eq. 1.31 has an appealing interpretation as a generalization of the discrete spatial Wiener filter (DSWF) [40]. Multiplying by $\mathrm{U}_{\Psi}{ }^{\mathrm{H}}$ rotates the problem into the coordinate frame where the spatial components are uncorrelated. Each component is then weighted by the Wiener gain for the ratio of the mode power to the noise power for that component $\sigma_{\Psi m}^{2} \sigma_{d}^{2} /\left(\sigma_{\Psi m}^{2} \sigma_{d}^{2}+\sigma_{n}^{2}\right)$. These estimates of the components are then multiplied by the inverse singular values $\sigma_{\Psi m}^{-1}$ before being transformed into mode coefficients by $\mathrm{V}_{\Psi}$. For the case when all the singular values are $1, \mathrm{~V}_{\Psi}=\mathrm{I}$ and $\mathrm{U}_{\Psi}$ is the appropriate set of samples of complex exponentials, Eq. 1.31 reduces exactly to the DSWF. 
Eq. 1.31 provides theoretical justification for the pragmatically-motivated mode filtering algorithm proposed by T. C. Yang in [41]. Yang's algorithm suggested deleting very small eigenvalues of $\Psi^{\mathrm{H}} \Psi$ before inverting this product for the pseudoinverse mode filter. His paper proposes this approach to ensure numerical stability of the inverse and uses some rough a priori assumptions about the values of the mode coefficients. His approach is a limiting case of Eq. 1.31 as some of the singular values of $\Psi$ get very small compared to $\sigma_{d}$ and $\sigma_{n}$. Under these conditions, Yang's ad hoc "dropped eigenvalue" method is an approximation to the MAP mode filter obtained when the array gives a poorly-conditioned sampling of the mode shapes and some of the diagonal elements in Eq. 1.31 approach zero.

If the Kuperman-Ingenito noise model applies, and the process generating the modes has diagonal covariance $K_{\mathrm{dd}}$ with elements $\sigma_{d_{1}}^{2}, \ldots, \sigma_{d_{M}}^{2}$, the MAP estimator is

$$
\begin{aligned}
\hat{\mathbf{d}}_{\mathrm{MAP}}= & {\left[K_{\mathrm{dd}}^{-1}+K_{\tilde{\mathrm{d}} \tilde{\mathrm{d}}}^{-1}\right]^{-1} K_{\tilde{\mathrm{d}} \mathbf{\mathrm { d }}}^{-1} \Psi^{\dagger} \mathbf{p} } \\
= & {\left[\begin{array}{cccc}
\frac{\sigma_{d_{1}}^{2}}{\sigma_{d_{1}}^{2}+\sigma_{\dot{d}_{1}}^{2}} & 0 & \cdots & 0 \\
0 & \frac{\sigma_{d_{2}}^{2}}{\sigma_{d_{2}}^{2}+\sigma_{\dot{d}_{2}}^{2}} & \ddots & \vdots \\
\vdots & \ddots & \ddots & 0 \\
0 & \cdots & 0 & \frac{\sigma_{d_{M}}^{2}}{\sigma_{d_{M}}^{2}+\sigma_{\tilde{d}_{M}}^{2}}
\end{array}\right] \Psi^{\dagger} \mathbf{p} . }
\end{aligned}
$$

Because both the signal and noise processes are independent random variables transformed by the same linear transformation $(\Psi)$, multiplying $\mathbf{p}$ by the pseudo-inverse performs the discrete spatial Karhunen-Loeve transform, decorrelating the mode estimation problem into $M$ independent problems. Unlike the spatially white noise model, the physical basis of interest (mode space) coincides with the mathematical basis in which the underlying processes are independent. The diagonal elements in Eq. 1.32 become the Wiener gains for each of the independent components. Thus 
the structure of Eq. 1.32 shows that the estimation problem becomes $M$ independent MAP problems once $\mathrm{p}$ is multiplied by $\Psi^{\dagger}$. When the random processes have probability density functions which are symmetric about their means, such as the Gaussian distribution, Eqs. 1.31 and 1.32 are also the minimum mean-squared error (MMSE) solutions [35].

\subsubsection{Relation of Mode Filters to Target Pressure Vector}

As mentioned earlier, the variety of mode filtering algorithms corresponds to a variety of choices for the target pressure vector for the control algorithm. It is possible to formulate the control problem in terms of the desired pressure field rather than the desired mode coefficients. Section 2.2 discusses the circumstances when it is preferable to use this approach. The control algorithm uses an estimate of how the pressure field observed at the hydrophone $\mathbf{p}$ is a function of the source array weight vector $\mathbf{w}$ to choose $\mathbf{w}$ to obtain some target pressure vector $\mathbf{p}_{d}$. Formulating the problem as a mode filtering problem at the feedback array gives insight into the best choice of $\mathbf{p}_{d}$. The target pressure vector should be the pressure vector which would yield the desired mode coefficient vector $\mathbf{d}_{d}$ when the most appropriate mode filter for the ocean environment and feedback array geometry is applied.

The most obvious choice for $\mathrm{p}_{d}$ is $\Psi \mathrm{d}_{d}$. This target pressure vector corresponds to the pseudo-inverse mode filter, since $\Psi^{\dagger} \mathbf{p}_{d}=\Psi^{\dagger} \Psi \mathrm{d}_{d}=\mathrm{d}_{d}$. Thus, if this choice for $\mathbf{p}_{d}$ were observed at the feedback array, the pseudo-inverse mode filter would indicate the pressure consisted of the desired modes. Alternatively, the choice $\mathbf{p}_{d}=\Psi\left(\Psi^{\mathrm{H}} \Psi\right)^{-1} \mathrm{~d}_{d}$ corresponds to the sampled mode shape filter, since $\Psi^{\mathrm{H}} \mathbf{p}_{d}$ would give $\Psi^{\mathrm{H}} \Psi\left(\Psi^{\mathrm{H}} \Psi\right)^{-1} d_{d}=\mathrm{d}_{d}$. Similar expressions can be derived for the MAP mode filters with the various noise models.

The algorithms described in this thesis will use $\mathrm{p}_{d}=\Psi \mathrm{d}_{d}$. The feedback array is assumed to sample the channel sufficiently that the pseudo-inverse mode filter is the best choice of mode filter. This is a reasonable assumption for the relatively 
shallow water environments and mid-frequency transmissions examined in this thesis. Also, over the relatively short range between the source array and feedback array, the signal-to-noise (SNR) ratio at the feedback array should be high if the signal is going to be observable at the distant observation array as shown in Fig. 1-1. At high SNR, the MAP formulations reduce asymptotically to $\mathrm{p}_{d}=\Psi \mathrm{d}_{d}$. If these favorable conditions do not apply, the target pressure vector should be chosen based upon the appropriate mode filtering algorithm for the environment and geometry, but generally this means the algorithm will not be able to produce a single mode with high fidelity.

\subsection{Control Theory}

The field of control theory provides some important insights into the single mode excitation problem. The classification of control systems as either open-loop and closed-loop systems is one of the fundamental distinctions in control theory [42]. Both kinds of systems use a control law for determining the control input to attempt to attain the desired behavior of the output of the plant, or system controlled. For the mode excitation problem, the input is the source array weights, w, the plant is the ocean between the source array and the feedback array, the output is the samples of the pressure field observed at the feedback array, $\mathbf{p}$, and the desired behavior is the target pressure $\mathbf{p}_{d}$. For an open-loop system, the input is chosen based solely on a priori or assumed knowledge of the system. All prior work on single mode excitation used open-loop control [15], [16]. The behavior of a plant controlled with this approach can be very sensitive to inaccuracies in this a priori information or in the model of the plant. The algorithm proposed in this thesis uses feedback control to obtain the desired performance. Feedback control modifies the input to the plant based on the difference between the observed and desired output of the plant. This allows the control system to compensate for inaccuracies in the a priori information 
about the plant as well as respond to time-variations in the plant. Figs. 1-2 and 1-3 below illustrate the two approaches for the single mode excitation problem.

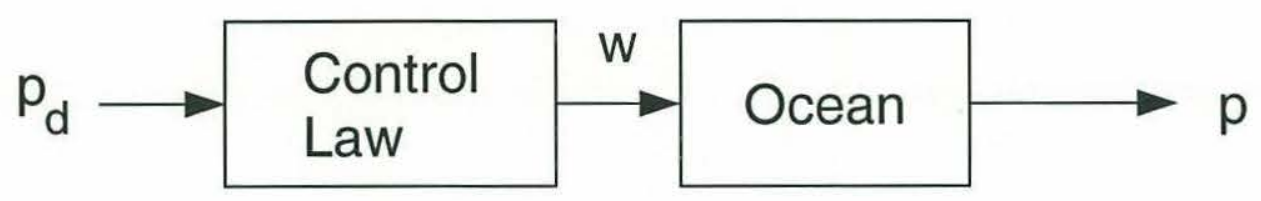

Figure 1-2: Open-loop Control For Single Mode Excitation

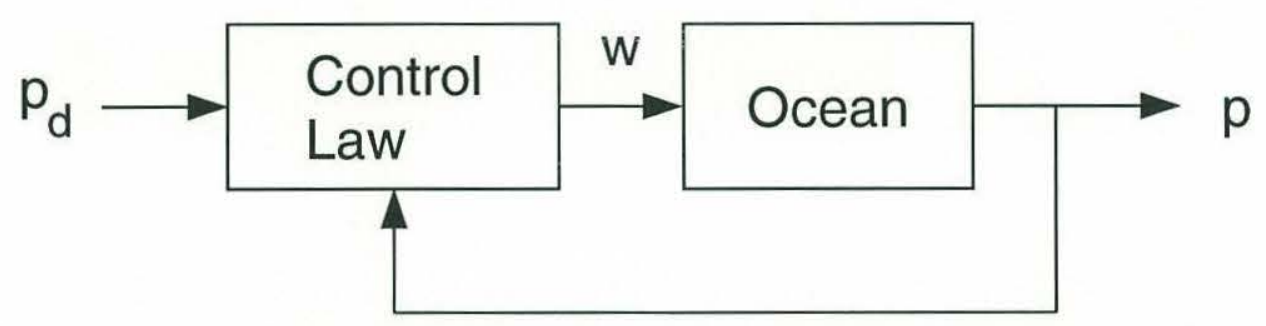

Figure 1-3: Closed-loop Control For Single Mode Excitation

Another useful concept from control theory is the use of state space models for systems with complicated but linear dynamics. Many feedback control algorithms use such a model to estimate the behavior of a plant they wish to control. Applying this model to the acoustic propagation between the source and feedback arrays provides important insight into several aspects of the single mode excitation problem. For the discrete-time case, the state-space model describes the evolution of the $1 \times M$ state vector $\mathbf{x}[n]$ by

$$
\mathbf{x}[n]=\mathbf{A}[n-1] \mathbf{x}[n-1]+\mathbf{B}[n] \mathbf{u}[n]+\mathbf{f}[n]
$$

where $\mathbf{u}[n]$ is the $1 \times L$ input vector for the system, and $\mathbf{f}[n]$ is a zero-mean white Gaussian noise with covariance $\mathbf{P}_{\mathrm{ff}}$ which is uncorrelated with all other signals in the problem. The output of the system $\mathbf{y}[n]$ is a $1 \times N$ vector

$$
\mathbf{y}[n]=\mathbf{C}[n] \mathbf{x}[n]+\mathbf{D}[n] \mathbf{u}[n]+\mathbf{n}[n],
$$


where $\mathbf{n}[n]$ is zero-mean, white Gaussian observation noise uncorrelated with all other signals in the problem, with covariance $\mathbf{P}_{\mathbf{n n}}$. Defining the state vector $\mathbf{x}[n]$ as the modes $\mathbf{d}[n]$ present at the feedback array at the $n^{\text {th }}$ iteration, the input as $\mathbf{w}[n]$, the complex source weights, and the output $\mathbf{y}[n]$ as $\mathbf{p}[n]$, the pressure observed at the feedback array, the state space model for the modal propagation between the source and feedback arrays simplifies to

$$
\begin{aligned}
& \mathbf{d}[n]=\mathbf{B}[n] \mathbf{w}[n]+\mathbf{f}[n] \\
& \mathbf{p}[n]=\Psi[n] \mathbf{d}[n]+\mathbf{n}[n] .
\end{aligned}
$$

In these equations, the $\mathbf{B}[n] \mathbf{w}[n]$ term is the portion of the total pressure field excited the source array, the $\mathbf{f}[n]$ term is the propagating background noise which satisfies the Kuperman-Ingenito noise model, and $\mathbf{n}[n]$ represents the sensor noise. The development that follows does not fully exploit this general model, but it is a conceptually appealing model for scenarios when there are different noise sources with differing spatial covariance structures. The matrix $\mathbf{B}[n]$ incorporates the effects of the mode shapes at the source array on the energy initially excited in each mode (Eq. 1.7), as well as any coupling of energy among the discrete modes between the source and feedback arrays. Note that Eq. 1.36 has the same form as the mode filtering problem (Eq. 1.15). Modeling the modal propagation in this way allows the application of several powerful results from control theory to the single mode excitation problem.

Reachability and observability are two control theory concepts germane to the mode excitation problem as formulated above in Eqs. 1.35 and 1.36. A system is said to be reachable over the interval $\left[n_{0}, n_{1}\right]$ if it is possible to drive the system to any final state $\mathbf{x}\left[n_{1}\right]$ from any initial state $\mathbf{x}\left[n_{0}\right]$ using $\mathbf{u}[n]$. The effect of the input $\mathbf{u}[n]$ on the state $\mathbf{x}\left[n_{1}\right]$ can be written as $\mathbf{\Phi}\left[n_{1}, n\right] \mathbf{B}[n] \mathbf{u}[n]$, where $\boldsymbol{\Phi}\left[n_{1}, n\right]$ is the state-transition matrix from time $n$ to $n_{1}$ for the undriven system. In the absence of inputs, $\mathbf{x}\left[n_{1}\right]=\Phi\left[n_{1}, n\right] \mathbf{x}[n]$. The total effect of the input over the interval $\left[n_{0}, n_{1}\right]$ 
on the state at $n_{1}$ can be written as

$$
\mathbf{x}\left[n_{1}\right]=\left[\mathbf{B}\left[n_{1}\right]\left|\mathbf{\Phi}\left[n_{1}, n_{1}-1\right] \mathbf{B}\left[n_{1}-1\right]\right| \ldots \mid \mathbf{\Phi}\left[n_{1}, n_{0}\right] \mathbf{B}\left[n_{0}\right]\right]\left[\begin{array}{c}
\frac{\mathbf{u}\left[n_{1}\right]}{\mathbf{u}\left[n_{1}-1\right]} \\
\frac{\mathbf{u}\left[n_{0}\right]}{\vdots}
\end{array}\right.
$$

For a system to be reachable, the partitioned matrix containing the B and $\boldsymbol{\Phi}$ terms must have full rank. The controllability gramian $\mathbf{W}_{1}\left[n_{0}, n_{1}\right]$ of a system is defined to be the correlation of this matrix. A system is reachable if and only if $\mathbf{W}_{1}\left[n_{0}, n_{1}\right]$ has full rank. For the simple state space model of Eqs. 1.35 and 1.36, the controllability gramian is

$$
\mathbf{W}_{1}\left[n_{0}, n_{1}\right]=\mathbf{B}\left[n_{1}\right] \mathbf{B}^{\mathrm{H}}\left[n_{1}\right],
$$

so the system is reachable over $\left[n_{0}, n_{1}\right]$ if and only if $\mathbf{B}\left[n_{1}\right]$ has full row rank $\mathbf{M}$. $\mathbf{A}$ system is defined to be completely reachable if it is reachable over some interval, and is uniformly completely reachable if there exists some $\alpha$ and $m$ such that the smallest eigenvalue of $\mathbf{W}_{1}[n, n+m]$ is greater than $\alpha$ for all $n$. Because the modes present at the feedback array for this model depend only on the input weights from the current iteration, properties like uniform complete reachability depend solely on $\mathbf{B}[n]$.

A system is described as observable over the interval $\left[n_{0}, n_{1}\right]$ if the state $\mathbf{x}\left[n_{0}\right]$ can be uniquely determined from knowing $\mathbf{u}[n]$ and $\mathbf{y}[n]$ over this interval. It can be shown this property is equivalent to the observability gramian $\mathbf{M}\left[n_{0}, n_{1}\right]$ having full rank $M$ [43]. The very simple state space model of mode propagation has the following observability gramian:

$$
\mathbf{M}\left[n_{0}, n_{1}\right]=\Psi^{\mathrm{H}}\left[n_{0}\right] \Psi\left[n_{0}\right] .
$$


Thus, the coefficients of the modes present at the feedback array can only be uniquely determined if the sampled mode shape matrix $\Psi_{\mathbf{r}}[n]$ has full column rank.

Framing the mode excitation problem in the state space model clearly separates the two primary issues of the problem. The first issue is whether it is possible to excite the desired modes in the channel. The second issue is whether the control algorithm can recognize the desired mode profile by observing samples of the pressure field. The first issue corresponds to the reachability of the system as described by the state space model for mode propagation. The reachability depends on the source array geometry and the propagation conditions between the source array and feedback array. For the ranges and depths discussed in this thesis, the propagation is usually predominantly determined by the bottom composition and bathymetry. Since the source array is the only factor effecting reachability under the control of the scientist, care must be taken to design the source array to ensure reachability over as wide a range of ocean conditions as possible.

The ability of the algorithm to recognize the desired mode profile from pressure observations corresponds to the observability of the system in the state space formulation. The observability of the system depends on the mode shapes and feedback array geometry. The mode shapes are a function of the sound speed profile and bottom properties at the array location, and are not under the control of the observer. Thus, the feedback array must be designed carefully to guarantee observability over as wide a range of sound speed profiles and bottom properties as possible to ensure the experiment's success.

An informative experiment and persistency of input are two additional important concepts from system identification and control theory. An experiment is said to be informative if it allows the unique identification of a single set of system model parameters out of the class of proposed system models [44]. A necessary condition for an experiment to be informative is that the control input sequence be persistent. A persistent input sequence is one whose autocorrelation matrix has full rank. This 
criteria will be formulated more precisely in Chapter 2 where the class of channel models used by the control algorithm is presented. The conflicting requirements of keeping the source weights fixed to obtain a high fidelity mode and varying them enough to get a persistent input for system identification will be an important issue for the control algorithm to address.

\subsection{Matched Signals}

Single mode excitation at a feedback array location can be viewed as a generalization from the time domain to the mode domain of the matched signal problem addressed by Parvulescu and Clay [45], [46], [47]. Parvulescu and Clay investigated the time-domain dispersion due to propagation through underwater acoustic channels. Specifically, they were interested in finding a signal which when transmitted from a single source through a given channel would give an "impulse-like" signal at a single receiving hydrophone. They observed that when they transmitted a short impulsive waveform, the received signal exhibited the well-known multi-path arrival structure. The matched filter whose impulse response is the time-reversed version of the received signal is the best linear detector for this signal in the presence of white noise [35]. Parvulescu and Clay noted that if the transmitted signal is a time-reversed version of the channel impulse response, the acoustic propagation will perform the matched filtering operation, and the received signal should be temporally concentrated. No matched filter will be necessary at the receiver. They successfully implemented a simple version of such a system using a reel-to-reel tape deck and two ships about $18 \mathrm{~km}$ apart in $2 \mathrm{~km}$ of water connected by a radio link. The source ship transmitted an impulsive signal, and the signal observed at the receiving ship was recorded on the tape deck over the radio channel. The source ship then transmitted a time-reversed version of the recorded impulse response by playing the tape backwards, and the received waveform was the desired temporally-concentrated sig- 
nal. In addition, by making several successive transmissions using the same reversed impulse response, Parvulescu and Clay attempted to estimate the coherence time of the channel from the deterioration of the received impulse. Unfortunately, they could not separate the effects of channel variability from those due to the source ship drifting. The combination of these effects resulted in the received signal being almost completely decorrelated with the matched filter after about 30 minutes. Because the source ship had drifted approximately $500 \mathrm{~m}$ over this time interval, Parvulescu and Clay proposed this displacement was the primary factor causing the mismatch, but acknowledge without a rigorous control for the experiment it would be impossible to say conclusively.

DiNapoli et al. attempted a similar experiment over a much longer range (50$150 \mathrm{~km})$ and lower frequencies $(10-30 \mathrm{~Hz})$ in the Arctic [48]. Because the Arctic environment is fairly stable, they believed computational models could predict the impulse response of the propagation path, and no attempt was made observe the actual impulse response as Parvulescu and Clay did. The transmissions DiNapoli et al. made using a reversed version of the impulse response predicted by computational models failed to give a clearly identifiable, temporally concentrated arrival. The most likely causes for this disappointing result are inaccuracies in their environmental knowledge and in the Arctic acoustic models used to predict the impulse response. The impulsive nature of noise in the Arctic due to ice dynamics also would make it difficult to identify the desired arrival amidst the ice noise.

The single mode excitation problem generalizes this approach from attempting to create a waveform which is concentrated over a short time interval one which is concentrated at a single mode. The challenge is to determine which transmitted source array weights will give the desired mode coefficients at the feedback array. If the channel is assumed to be range-invariant, the source array weights exciting a single mode can be computed from the sound speed profile and bottom composition at the source array. This is analogous to assuming the channel is dispersionless and 
has no multi-path interference for the time-domain experiments of Parvulescu and Clay. Most shallow water channels are not range-invariant. In many range-varying channels, the weights computed assuming the channel is range-invariant will excite a variety of modes at the feedback array location, and not just the desired single mode. The source initially excites a single mode which is then distorted by propagation through the channel, coupling its energy into several modes. The question then arises how best to excite an initial distribution of modes at the source array which is pre-compensated for this distortion such that the coupling caused by the channel will cancel out all the modes except the desired one when the pressure field is observed at the feedback array. The feedback control algorithm attempts to estimate the matched signal for the channel coupling between the source and feedback arrays adaptively. This multiple input/multiple output problem is more complicated than the single source/single hydrophone one addressed by Parvulescu and Clay, but the underlying strategy of matching the transmitted signal to the environment is the same.

\subsection{Summary}

As noted at the start of this chapter, the primary contribution of this thesis is the synthesis of ideas from several disciplines to produce a new algorithm for exciting a single mode. This chapter has reviewed the relevant background information that will be used to derive the control algorithm in the next chapter. The section on mode filtering yielded an important perspective on the relationship among the MAP, pseudo-inverse, and sampled mode shape mode filters. The MAP filter was also shown to provide a theoretical justification for Yang's ad hoc dropped eigenvalue mode filter. Chapter 2 will show how observability and reachability can provide insight into how the source and feedback array geometries affect the ability of the algorithm to excite a single mode. 


\section{Chapter 2}

\section{Control Algorithm}

This chapter presents an algorithm for single mode excitation using indirect control [14]. An indirect control algorithm explicitly estimates a model of the plant, then uses this model to compute the control input giving the desired behavior. The algorithm proposed for single mode excitation begins with some initial estimate of the acoustic propagation through the feedback volume. Based on this estimate, it computes the best set of source array weights $\mathbf{w}$ to get the desired pressure vector $\mathbf{p}_{d}$. After exciting the channel using $\mathbf{w}$, the algorithm compares the observed pressure vector $\mathbf{p}$ to $\hat{\mathbf{p}}$, the pressure predicted by the current channel estimate. The channel estimate is updated based on the difference between the observed and predicted pressure, and the process begins again. Figure 2-1 shows the result of incorporating this type of control algorithm into the scenario of Figure 1-1. While this control scheme is fairly simple, it clearly demonstrates the potential of feedback control for exciting a single mode. If this straight-forward scheme proves successful, more advanced control schemes like robust control [49] [50] [51] can be investigated for this problem.

The control algorithm uses the replica matrix described in Section 2.1 to model the propagation between the source and feedback arrays. Section 2.1 also presents a more precise definition of persistent excitation as first discussed in Section 1.4. The 


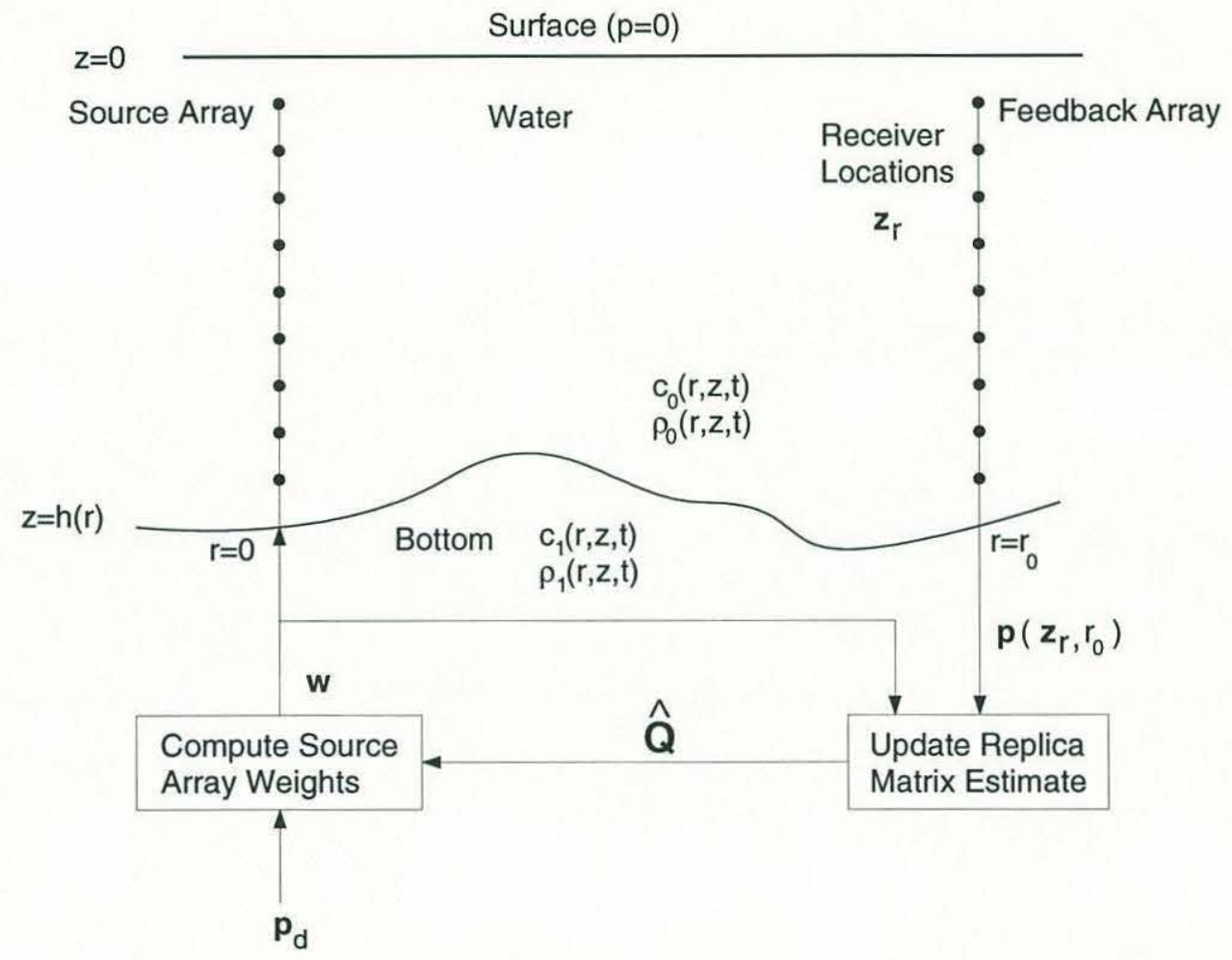

Figure 2-1: Control Algorithm for Ocean Experiment 
source array weights are computed from the current estimate of the channel replica matrix using the least squares method described in Section 2.2. The replica matrix estimator is an important part of the control algorithm, and this chapter presents three different estimators for the replica matrix. Section 2.3 develops the condition number-limited least squares algorithm for estimating the channel replica matrix. The replica matrix estimation problem can also be formulated as a Kalman filtering problem, and Section 2.4 presents the resulting replica matrix estimator. Section 2.5 develops the least mean squares (LMS) [13] estimator for the replica matrix. This common adaptive algorithm will be compared with the other two estimation algorithms in the experimental work of Chapter 4.

\subsection{Propagation Model}

The control algorithm presented in this chapter models the pressure received at the feedback array $\mathbf{p}$ as a linear function of the complex source array weights $\mathbf{w}$, i.e.,

$$
\mathrm{p}=\mathrm{Qw}
$$

where $\mathbf{Q}$ is the replica matrix for the channel. The $\ell^{\text {th }}$ column of $\mathbf{Q}$ is the replica vector at the feedback hydrophone array for the $\ell^{\text {th }}$ source array element. Equivalently, the $(i, j)^{\text {th }}$ element of $\mathrm{Q}$ is the Green's function between the $j^{\text {th }}$ source and $i^{\text {th }}$ receiver evaluated at the transmission frequency. Considering the state space models of Eqs. 1.35 and 1.36, the replica matrix is $\mathbf{Q}[n]=\Psi_{r}[n] \mathbf{B}[n]$. Thus, this model combines the effects of the feedback array hydrophone locations on $\Psi_{r}[n]$ with the transmission properties of $\mathbf{B}[n]$ summarizing the source locations and mode coupling. Poor conditioning in $\mathbf{Q}[n]$ can be caused by any or all of the following phenomena: poor hydrophone sampling of the modes at the feedback array, poor placement of the source array elements for exciting the modes at the source array, or unfavorable mode coupling between the source and feedback arrays. 
The validity of $\mathrm{Q}[n]$ as a propagation model depends on the time scales of variations in the channel relative to the propagation time between the source and feedback arrays. As noted in Section 1.2, the replica matrix model is valid for continuous wave $(\mathrm{CW})$ propagation in linear, time-invariant propagation environments. For the typical scenario shown in Fig. 1-1, the propagation time from source to feedback array is 1-2 sec. The oceanographic features and processes dominating shallow water acoustics such as internal waves, tidal mixing, and bottom bathymetry are believed to vary on timescales much longer than this. Observations in the recent SWARM experiment indicated the predominant energy in the internal waves in a typical coastal environment had timescales on the order of five to seven minutes [52]. Based on these observations, it appears safe to assume the channel is time-invariant over the propagation interval between the source and feedback arrays.

The iteration time for the algorithm will depend both on the distance $r_{0}$ from the source to feedback array and the group velocities of the modes as determined by the sound speed profile $c(r, z)$. After changing the source array weights, the control algorithm must wait long enough for the slowest mode to propagate from the source array to the feedback array before observing the pressure field and updating its estimate of the channel replica matrix. The control algorithm samples the propagation environment with sampling period equal to its iteration time. The Nyquist sampling theorem [53] indicates that setting the iteration time to be at least the propagation time for the slowest mode means that oceanographic processes at timescales shorter than twice this propagation time will be aliased in the observations of the environment. This best case limit on the bandwidth of observable processes must be taken into account when designing the experiment. Due to the averaging and windowing of data employed by the estimators described in this chapter, the limit on the fastest processes the control algorithm can expect to track is much slower than the limit specified by the Nyquist sampling theorem.

A sequence of inputs $\mathrm{w}[0], \ldots, \mathrm{w}[n]$ is said to be persistent if it allows unique 
identification of one set of model parameters out of all possible models in a given model class [44]. Since the replica matrix model for propagation defines the space of possible models to be all $N \times L$ complex matrices $\left(\mathcal{C}^{N \times L}\right)$, persistency can be defined more precisely than the general discussion in Section 1.4. To identify a unique $\mathbf{Q}$, the sequence of input vectors must span $\mathcal{C}^{L}$. Thus, persistency over the interval $[0, n]$ is equivalent to $[\mathbf{w}[0]|\mathbf{w}[1]| \ldots \mid \mathbf{w}[n]]$ having rank $L$. This condition is also equivalent to the square matrix $\sum_{i=0}^{n} \mathbf{w}[i] \mathbf{w}^{\mathrm{H}}[i]$ having full rank.

Because the control algorithm attempts to hold the pressure field observed at the feedback array fixed, the input signal usually will not vary after an initial convergence transient if the replica matrix estimate $\hat{\mathbf{Q}}$ is accurate and the channel is quasi-stationary. Later discussions will illustrate how this impersistent or nearly impersistent input sequence affects the convergence and transient behavior of the estimators. If the steady state source array weights $\mathbf{w}_{s s}$ are orthogonal to the errors in the rows of $\hat{\mathbf{Q}}[n]$, these errors will not be revealed by the algorithm in the steady state. Although the errors in $\hat{\mathbf{Q}}$ are undetectable by $\mathbf{w}_{s s}, \mathbf{w}_{s s}$ may not be the source array weight vector giving the best performance. The control algorithm does have fixed points other than the global optimum $\mathbf{w}_{L S}=\mathbf{Q}^{\dagger} \mathbf{p}_{d}$, and thus may converge to a non-optimal solution for $\mathrm{w}$. In the simulations and experiments described later in this thesis, the performance of the algorithm was limited by the observation noise, and not by convergence to non-optimal solutions. The fixed points at these non-optimal solutions are generally unstable equilibria, so observation noise should provide sufficient impetus to propel the control algorithm away from them. This likely explains why no evidence was observed of the algorithm getting stuck at nonoptimum source weights in the simulations or laboratory waveguide experiments. 


\subsection{Source Array Weight Selection}

The least squares solution to the system of linear equations

$$
\mathbf{p}_{d}=\mathrm{Qw}
$$

yields the best source array weight vector for obtaining the desired pressure $\mathbf{p}_{d}$ given the current replica matrix $\mathrm{Q}$. Assuming $N>L$, the singular value decomposition of $\mathbf{Q}$ is

$$
\mathbf{Q}=\mathrm{U}_{\mathbf{Q}}\left[\begin{array}{ccc}
\sigma_{\mathrm{Q} 1} & & 0 \\
& \ddots & \\
0 & & \sigma_{\mathrm{Q} L} \\
\hline & 0 &
\end{array}\right] \mathrm{V}_{\mathrm{Q}}{ }^{\mathrm{H}}
$$

where $\mathrm{U}_{\mathbf{Q}}$ is $N \times N, \mathbf{V}_{\mathbf{Q}}$ is $L \times L$, the partitioned matrix on the right-hand side is $N \times L$, and the diagonal elements of that matrix are ordered such that $\sigma_{\mathbf{Q} 1} \geq \sigma_{\mathbf{Q} 2} \geq \ldots \geq \sigma_{\mathbf{Q} L} \geq 0$ [37]. The pseudo-inverse of $\mathbf{Q}, \mathbf{Q}^{\dagger}$, is defined to be

$$
\mathbf{Q}^{\dagger}=\mathbf{V}_{\mathbf{Q}},\left[\begin{array}{ccc|c}
\sigma_{\mathrm{Q} 1}^{-1} & & 0 \\
& \ddots & & 0 \\
& & & \\
0 & & \sigma_{\mathrm{Q} L}^{-1} &
\end{array}\right] \mathrm{U}_{\mathbf{Q}}{ }^{\mathrm{H}}
$$

except that any $\sigma_{\mathbf{Q} i}$ 's equal to zero are not inverted, but left as zero. If all the singular values $\sigma_{\mathbf{Q} i}$ are non-zero, $\mathbf{w}=\mathbf{Q}^{\dagger} \mathbf{p}_{d}$ gives the unique source array weight vector minimizing the error between the desired and obtained pressures. If the propagation is completely described by $M$ trapped modes, and $M<L$, there will be singular values of $\mathbf{Q}$ that are zero, and the solution minimizing the magnitude of the error is not unique. In this case, $\mathbf{w}=\mathrm{Q}^{\dagger} \mathbf{p}_{d}$ gives the minimum norm source array 
weight vector with the minimum squared error between the observed pressure $\mathbf{p}$ and the desired pressure $\mathbf{p}_{d}$ for the true channel replica matrix $\mathbf{Q}$. The weight selection step uses the same method to find $\mathrm{w}$ except $\mathrm{Q}$ is replaced by the current replica matrix estimate $\hat{\mathbf{Q}}$. For the $n^{\text {th }}$ iteration, the source array weights are determined by

$$
\mathbf{w}[n]=(\hat{\mathbf{Q}}[n])^{\dagger} \mathbf{p}_{d}
$$

where $\hat{\mathbf{Q}}[n]$ is the estimate of the channel replica matrix at the $n^{\text {th }}$ iteration. This gives the source array weight vector that would be the best fit if the current channel estimate $\hat{\mathbf{Q}}[n]$ were correct.

The weight selection step proposed in Eq. 2.5 minimizes the error in the pressure domain, which does not generally give the minimum error in the mode domain. It is possible to compute $\mathrm{w}$ to minimize the error in the mode domain using an estimate of $\mathbf{B}[n]$ from the state-space model of Eq. 1.35. The weight selection step then uses solves Eq. 1.35 in the least-squares sense assuming the state noise $\mathbf{f}[n]$ is zero. The resulting source array weight vector

$$
\mathbf{w}[n]=(\hat{\mathbf{B}}[n])^{\dagger} \mathbf{d}_{d}
$$

should minimize the error in the mode domain. In practice, this approach can be problematic for numerical reasons discussed below.

It is necessary to develop a precise framework to discuss the important issues that arise when some of the matrices in the control algorithm computations are not mathematically singular, but have very large condition numbers. Golub and Van Loan define the $\epsilon$-rank, $r_{\epsilon}$, of the matrix $\mathrm{Q}$ to be the number of singular values $\sigma_{i} \geq \epsilon$, i.e., if $r_{\epsilon}=\operatorname{rank}(\mathbf{Q}, \epsilon)=m$, then $\sigma_{\mathbf{Q} 1} \geq \sigma_{\mathbf{Q} 2} \geq \ldots \geq \sigma_{\mathbf{Q} m} \geq \epsilon>\sigma_{\mathbf{Q} m+1} \geq \sigma_{\mathbf{Q} L} \geq 0$ [37]. It is straight-forward to extend this idea and define the $\epsilon$-span of a matrix with $\epsilon$-rank $r_{\epsilon}$ as the span of $\left\{\mathbf{u}_{1}, \ldots, \mathbf{u}_{r_{\epsilon}}\right\}$, where the $\mathbf{u}_{i}$ are the columns of $\mathbf{U}_{\mathbf{Q}}$, the left orthogonal matrix of the SVD. Similarly, the $\epsilon$-nullspace of the matrix can be defined 
to be the space spanned by $\left\{\mathbf{v}_{r_{\epsilon}+1}, \ldots, \mathbf{v}_{L}\right\}$, where the $\mathbf{v}_{i}$ 's are the columns of $\mathbf{V}_{\mathbf{Q}}$, the right orthogonal matrix of the SVD.

If $\hat{\mathbf{Q}}[n]$ has $\epsilon$-rank less than $M$ for some $\epsilon$ appropriate to the scale of the experiments, it indicates the system is either numerically unreachable or unobservable, either of which may be problematic for mode excitation. The terms nearly unreachable and nearly unobservable will be used to describe these numerically troublesome conditions. If the system is nearly unreachable, both the pressure and mode domain approaches for computing $\mathbf{w}[n]$ may have difficulty exciting the desired mode. In practical terms, this condition corresponds to the scenario where the source element locations and propagation conditions in the feedback volume combine unfavorably so that some modes cannot be excited to any significant level given the power limitations on the sources. If the desired pressure $\mathbf{p}_{d} \in \epsilon$-span $\{\mathbf{Q}\}$, then the desired pressure field may be obtained within the power limits of the practical system as reflected by the choice of $\epsilon$. Otherwise, the power required to excite this particular mode exceeds the limitations of the sources. It is possible that for a given source array geometry and propagation conditions which result in numerical unreachability, some modes will still be excitable and others will not, depending on the $\epsilon$-span of $\mathbf{Q}$.

If the $\epsilon$-rank deficiency of $\hat{\mathbf{Q}}[n]$ is because the system is nearly unobservable, there may be undesired trapped modes propagating at the feedback array even when the observed pressure $\mathbf{p}[n]$ is a close fit to the desired pressure $\mathbf{p}_{d}$. Numerical unobservability can be due to one or more causes. Some modes may be sampled at or close to a pressure null, so that any energy in these modes cannot be seen over the observation noise. Alternatively, if one the columns of the sampled mode shapes can be written as a linear combination of other columns, the pressure field cannot be uniquely resolved into modes. These conditions are equivalent to the poorly conditioned sampling for $\Psi$ discussed in Section 1.3. If the feedback array sufficiently samples the water column such that the system is observable, the issue is not as important. In this case, exciting a pressure field close to the desired $\mathbf{p}_{d}$ will correspond 
to a mode coefficient vector $\mathbf{d}[n]$ close to the desired $\mathbf{d}_{d}$.

The weight vector minimizing $\left\|\mathbf{p}_{d}-\mathbf{p}[n]\right\|$ will not generally minimize $\left\|\mathrm{d}_{d}-\mathrm{d}[n]\right\|$. The errors in the two domains are related by a linear but generally non-unitary transformation $\Psi$ :

$$
\mathbf{p}_{d}-\mathbf{p}[n]=\Psi\left(\mathrm{d}_{d}-\mathrm{d}[n]\right)
$$

To see why the minimum error in the pressure domain does not necessarily correspond to the minimum error in the mode domain, consider the extreme case when $\Psi$ possesses a non-trivial null-space, i.e., when the system is unobservable. Let $\mathbf{d}[n]$ be the mode vector minimizing the error in the mode domain, and $\mathbf{p}[n]$ be resulting pressure vector, i.e., $\mathbf{p}[n]=\Psi[n] \mathrm{d}[n]$. Any $\tilde{\mathrm{d}}[n]=\mathrm{d}[n]+\Delta \mathbf{d}$, where $\Delta \mathbf{d}$ is in the null-space of $\Psi$ will attain the same pressure domain error, but not minimize the mode domain error. The argument is somewhat more involved when $\Psi$ does not have a null-space, but so long as the singular values of $\Psi$ are not all equal, the problem exists. If the system is well-sampled such that the $\sigma_{i}$ 's of $\Psi$ are at least commensurate with each other if not equal, the weight vector giving the minimum error in the pressure domain will give a small if not minimum error in the mode domain. In the limiting case with the array spanning the entire water column as $N \rightarrow \infty$, the $\sigma_{\Psi i}$ 's approach one and the solution minimizing the error in one domain also minimizes it in the other. As noted in Section 1.3 the difficulty of observing the pressure in the bottom prevents the singular values from actually reaching one, but generally they will come very close to it.

To see more clearly the difficulty in explicitly estimating $\hat{\mathrm{d}}[n]$ when $\Psi$ is nearly singular, consider the scenario where the observation noise $\mathbf{n}$ has a Gaussian, spatially white component with variance $\sigma_{n}^{2}$, and the propagation physics ensure the magnitudes of all mode coefficients are bounded by some constant $d_{\max }$, i.e.,

$$
\max _{i, n}\left\|d_{i}[n]\right\|<d_{\max }
$$


If $\operatorname{rank}\left(\Psi, \sigma_{n} / \lambda_{d}\right) \neq \min (M, N)$, the observations $\hat{\mathrm{d}}[n]=\Psi^{\dagger} \mathbf{p}[n]$ will be dominated by noise. One or more of the $\sigma_{\Psi i}^{-1}$ on the diagonal of $\Psi^{\dagger}$ will be large enough that it will make the white noise component in the $\mathbf{v}_{i}$ direction larger than the coefficients of the propagating modes. This will slow the convergence of the algorithm since the estimator will have to average many observations of $\hat{\mathrm{d}}[n]$ to obtain an accurate estimate of $\mathbf{B}$ from these low signal-to-noise ratio observations. Because $\hat{\mathbf{B}}[n]$ will converge slowly and fluctuate significantly due to noise, the weight vector given by Eq. 2.6 will vary considerably. Under these conditions, the pressure vector observed at the hydrophone will be unsatisfactory for two reasons. Because the system is nearly unobservable, undesired modes may be propagating at the feedback array which are not visible over the observation noise. In addition, fluctuations in $\hat{\mathbf{B}}[n]$ will make $\mathbf{w}[n]$ and consequently $\mathbf{p}[n]$ vary significantly from one iteration to the next. The pressure field at the feedback array may not only contain undesired modes, but may not even consist of the same modes on successive iterations. For these reasons, the pressure at the feedback array will be a poor source signal for oceanographic observers further downrange at the observation array.

If the modes are nearly unobservable, formulating the problem in the pressure domain will not alter the fact that the pressure field may consist of many modes, rather than the desired single mode. If the observation noise $\mathbf{n}[n]$ is relatively weak compared to the pressure due to the propagating modes, the pressure observed at the feedback array will not vary much when $\mathrm{w}[n]$ stays fixed. Because the noise is not amplified as it was when estimating $\hat{\mathbf{d}}[n]$, the observations $\mathbf{p}[n]$ are more reliable, and the estimate $\hat{\mathbf{Q}}[n]$ will converge faster than $\hat{\mathbf{B}}[n]$ and be less sensitive to noise. In this case the pressure domain approach will give a pressure field at the feedback array which is at least consistent over time even if it does not give the desired single mode. Although from the perspective of the oceanographic observers a high-fidelity single mode is the best source, a consistent mode distribution of lower fidelity is preferable to the erratic mode coefficients obtained by computing $\mathbf{w}[n]$ from $\mathbf{B}[n]$ 
when $\Psi[n]$ is poorly-conditioned.

If the spread of singular values of $\Psi$ is such that the modes are practically observable, i.e., $\left(\operatorname{rank}\left(\Psi, \sigma_{n} / \lambda_{d}\right)=M\right)$, but the $\mathbf{w}[n]$ minimizing $\left\|\mathbf{p}_{d}-\mathbf{p}[n]\right\|$ gives unacceptably large errors in the mode domain, the weight selection step can be reformulated as a weighted least squares problem using an error metric where all components in the mode domain are equally weighted. The source array weights $\mathrm{w}$ are chosen to minimize

$$
\|\tilde{\mathbf{e}}[n]\|^{2}=\|\operatorname{Re}[n]\|^{2}=\left(\mathbf{p}[n]-\mathbf{p}_{d}\right)^{\mathrm{H}} \mathbf{R}^{2}\left(\mathbf{p}[n]-\mathbf{p}_{d}\right),
$$

where $\mathbf{R}$ is an $N \times N$ positive semi-definite Hermitian matrix. Choosing

$$
\mathbf{R}=\mathbf{U}_{\Psi}\left[\begin{array}{ccc|c}
\sigma_{\Psi 1}^{-1} & & 0 & \\
& \ddots & & 0 \\
0 & & \sigma_{\Psi M}^{-1} & \\
\hline & 0 & & 0
\end{array}\right] \mathrm{U}_{\Psi}{ }^{H}
$$

gives the weighted pressure domain error whose minimization corresponds to the minimum error in the mode domain. Note that $\mathbf{R}$ has the same orthogonal complement as $\Psi^{\dagger}$, indicating pressure domain errors orthogonal to the columns of $\Psi$ are irrelevant to this metric. This is reasonable since if $\Psi$ is accurate, errors in these directions could not be due to the modes and must be observational noise. Solving the resulting least squares problem gives the new criteria for selecting the source array weight vector:

$$
\mathbf{w}[n]=\left(\hat{\mathbf{Q}}^{\mathrm{H}}[n] \mathbf{R}^{2} \hat{\mathbf{Q}}[n]\right)^{\dagger} \hat{\mathbf{Q}}[n] \mathbf{R}^{2} \mathbf{p}_{d} .
$$


If the replica matrix estimate $\hat{\mathbf{Q}}[n]=\Psi \hat{\mathrm{B}}[n]$, Eq. 2.11 reduces to

$$
\mathbf{w}[n]=(\hat{\mathbf{B}}[n])^{\dagger}\left(\boldsymbol{\Psi}^{\dagger} \mathbf{p}_{d}\right)=(\hat{\mathbf{B}}[n])^{\dagger} \mathbf{d}_{d}
$$

which would be the weight vector minimizing the error in the mode domain if an explicit estimate of $\hat{\mathbf{B}}[n]$ were available to the weight selection step. The weight vector that minimizes the weighted pressure domain error defined with Eqs. 2.9 and 2.10 gives the desired weight vector minimizing the error in the mode domain.

This section has developed a method for estimating the best source array weight vector $\mathbf{w}[n]$ to excite the desired pressure profile. This estimate depends on the current estimate of the channel replica matrix $\hat{\mathbf{Q}}[n]$. The remaining three sections of the chapter develop three different methods for estimating $\hat{\mathbf{Q}}[n]$ for the feedback volume.

\subsection{Condition Number-limited Least Squares}

The source array weight vector $\mathrm{w}[n]$ is a function of the current estimate of the replica matrix, $\hat{\mathbf{Q}}[n]$. In order to determine $\mathbf{w}[n]$, the control algorithm must compute $\hat{\mathbf{Q}}[n]$, the estimate of $\mathbf{Q}$ at the $n^{\text {th }}$ iteration, from the information available about the channel, specifically, the sequence of source array weights $\mathbf{w}[0], \ldots, \mathbf{w}[n-1]$ and the pressures observed at the feedback array $\mathbf{p}[0], \ldots, \mathbf{p}[n-1]$. Each of the $N$ rows of

$$
\hat{\mathbf{Q}}[n]=\left[\begin{array}{c}
\hat{\mathbf{q}}_{1}[n] \\
\vdots \\
\hline \hat{\mathbf{q}}_{N}[n]
\end{array}\right]
$$

can be estimated independently of the other rows using the observed pressure at the hydrophone of the feedback array corresponding to that row of the replica matrix. 
If the rows of $\mathbf{Q}[n]$ are assumed to be constant, the sequence of source array weights and feedback array pressures form a system of linear equations:

$$
\left[p_{i}[0]|\ldots| p_{i}[n-1]\right]=\mathbf{q}_{i}[\mathbf{w}[0]|\ldots| \mathbf{w}[n-1]]
$$

or

$$
\mathbf{P}_{i}[n-1]=\mathbf{q}_{i} \mathbf{W}[n-1],
$$

where

$$
\begin{aligned}
\mathbf{P}_{i}[n-1] & =\left[p_{i}[0]|\ldots| p_{i}[n-1]\right] \\
\mathbf{W}[n-1] & =[\mathbf{w}[0]|\ldots| \mathbf{w}[n-1]] .
\end{aligned}
$$

The least squares solution to this system provides $\hat{\mathbf{q}}_{i}[n]$

$$
\hat{\mathbf{q}}_{i}[n]=\mathbf{P}_{i}[n-1] \mathbf{W}^{\mathrm{H}}[n-1]\left(\mathbf{W}[n-1] \mathbf{W}^{\mathrm{H}}[n-1]\right)^{-1} .
$$

This equation can be manipulated into a recursive form by following the development of the Recursive Least Squares (RLS) algorithm [54]. Although the matrix inversion lemma used in that algorithm is a tempting means of avoiding the inversion in Eq. 2.18, the lemma is too numerically sensitive to be useful for this problem in practice. The extreme conditioning of $\mathrm{W}[n-1] \mathrm{W}^{\mathrm{H}}[n-1]$ and the numerical issues associated with it will be discussed in more detail later in this section. Because $\mathbf{W}[n-1] \mathbf{W}^{\mathrm{H}}[n-1]$ is not a function of $i$, the row number, the matrix inverted is the same for each row of $\hat{\mathbf{Q}}$. This allows all rows of the replica matrix estimate to be computed simultaneously. The same algebraic manipulations used to derive the RLS algorithm in [54] yield a partially recursive form of the least squares estimator for the channel replica matrix: 


$$
\begin{aligned}
\hat{\mathbf{Q}}[n+1] & =\hat{\mathbf{Q}}[n]+\mathbf{a}[n] \mathbf{k}^{\mathrm{H}}[n] \\
\mathbf{a}[n] & =\mathbf{p}[n]-\hat{\mathbf{Q}}[n] \mathbf{w}[n] \\
\mathbf{k}[n] & =\Phi^{-1}[n] \mathbf{w}[n] \\
\mathbf{\Phi}[n] & =\sum_{i=0}^{n} \mathbf{w}[i] \mathbf{w}^{\mathrm{H}}[i]=\mathbf{\Phi}[n-1]+\mathbf{w}[n] \mathbf{w}^{\mathrm{H}}[n] .
\end{aligned}
$$

Although this estimator must invert $\Phi[n]$ for each iteration, it is recursive in the sense that computing $\hat{\mathbf{Q}}[n+1]$ requires only the current observations $(\mathbf{p}[n]$ and $\mathbf{w}[n]$ ), the previous estimate $\hat{\mathbf{Q}}[n]$ and the source weight correlation matrix $\boldsymbol{\Phi}[n]$, itself easily updated. The complete sequence of source array weights and feedback array pressures need not be saved. Note that $\mathbf{a}[n]$ is the error signal between the observed pressure and the pressure predicted by the current estimate $\hat{\mathbf{Q}}[n]$.

The algorithm must be initialized with a replica matrix $\hat{\mathbf{Q}}[0]$ and source weight correlation $\Phi[0]$. These can be obtained by sequentially turning on each element of the source array with gain 1 , then collecting the observed pressures into the columns of $\hat{\mathbf{Q}}[0]$ and setting $\Phi[0]=\mathrm{I}$. Alternatively, $\hat{\mathbf{Q}}[0]$ can be set equal to some a priori guess at the replica matrix, and $\Psi[0]=\delta \mathbf{I}$ for some small constant $\delta$.

In realistic ocean environments, $\mathbf{Q}$ will not usually be constant, but slowly varying. The estimator specified in Eqs. 2.19-2.22 can be modified to include an exponential forgetting factor $\gamma$ to depreciate older observations and allow the estimator to respond to changes in the ocean environment [54]. This forgetting factor can be incorporated into the estimator by modifying Eq. 2.22 to

$$
\Phi[n]=\gamma \Phi[n-1]+\mathbf{w}[n] \mathbf{w}^{\mathrm{H}}[n],
$$

where $0<\gamma \leq 1$. Multiplying by $\gamma$ at each iteration modifies the estimator to choose $\hat{\mathbf{q}}_{i}[n]$ to minimize $\sum_{\ell=0}^{n} \gamma^{n-\ell}\left\|p_{i}[\ell]-\hat{\mathbf{q}}_{i}[\ell] \mathbf{w}[\ell]\right\|^{2}$. This new error criteria reduces 
the importance of old observations until they are eventually insignificant. The best choice of $\gamma$ will depend on the bandwidth and intensity of the oceanographic processes affecting acoustic propagation and the iteration time of the control algorithm. Choosing $\gamma$ to be too small will not allow the estimator to average enough observations to reduce the effects of observation noise. Too large a choice for $\gamma$ will not allow the estimator to respond expeditiously to changes in the propagation conditions. The choice of $\gamma$ must compromise between these conflicting demands.

The discussion of persistency in Section 2.1 observed that single mode excitation will generally require $\mathbf{w}[n]$ to be constrained within a small region of $\mathcal{C}^{L}$. The condition for a persistent input sequence given there is equivalent to $\Phi[n]$ as defined in Eq. 2.22 having full rank. An impersistent input causes problems for this algorithm because as $\mathbf{w}[n]$ remains constant or nearly constant, $\Phi[n]$ will become poorly conditioned. This causes difficulty computing $\Phi^{-1}[n]$ before $\Phi[n]$ is mathematically singular.

The large condition number of $\Phi[n]$ will cause the estimator to become overly sensitive to observation noise even before problems computing the inverse arise. A large sample of noise may make the error vector $\mathbf{a}[n] \neq 0$ even when $\mathbf{Q}[n]$ is accurate. The resulting small perturbation in $\hat{\mathbf{Q}}[n]$ will cause a slight perturbation in $\mathbf{w}[n]$ away from the correct source array weight vector. As the subsidiary eigenvalues of $\Phi[n]$ grow arbitrarily small, even a minor perturbation in $\mathbf{w}[n]$ from its fixed value will cause $\mathbf{k}[n]$ to have a very large norm. This could result in a large correction to $\hat{\mathbf{Q}}[n]$, and subsequently a large error in $\mathbf{w}[n]$ and $\mathbf{p}[n]$ for the next iteration. Although the estimator will quickly recover from this noise-induced transient, these excursions produce undesirable spikes in the error between the desired and observed mode coefficients. These spikes detract from the feedback array's value as a single mode source to observers further downrange in the scenario of Figure 1-1.

These transients may be prevented by limiting the condition number of the inverse of $\boldsymbol{\Phi}[n]$ to be no greater than some threshold $\eta$. To define this condition number- 
limited inversion more precisely, let the eigenvalue decomposition of $\Phi[n]$ be

$$
\Phi[n]=\mathrm{V}_{\Phi}\left[\begin{array}{ccc}
\lambda_{\Phi 1} & & 0 \\
& \ddots & \\
0 & & \lambda_{\Phi L}
\end{array}\right] \mathrm{V}_{\Phi}{ }^{\mathrm{H}}
$$

where $\lambda_{\boldsymbol{\Phi} 1} \geq \lambda_{\boldsymbol{\Phi} 2} \geq \ldots \geq \lambda_{\boldsymbol{\Phi} L} \geq 0$. The condition number-limited inverse $\boldsymbol{\Phi}_{C L}^{-1}[n]$ is defined to be

$$
\Phi_{C L}^{-1}[n]=\mathrm{V}_{\Phi}\left[\begin{array}{cccc}
\lambda_{1}^{-1} & & & \\
& \tilde{\lambda}_{2}^{-1} & & \\
& & \ddots & \\
& & \tilde{\lambda}_{L}^{-1}
\end{array}\right] \mathrm{V}_{\Phi}{ }^{\mathrm{H}}
$$

where $\tilde{\lambda}_{i}=\max \left(\lambda_{i}, \lambda_{1} / \eta\right)$. This insures the condition number of $\Phi_{C L}^{-1}[n]$ is never greater than $\eta$, while $\lambda_{1}$, the eigenvalue corresponding to the predominant eigenvector, is correctly inverted. Using $\boldsymbol{\Phi}_{C L}^{-1}[n]$ to compute $\mathbf{k}[n]$ insures that no component of $\mathbf{w}[n]$ is unduly amplified, and thus the norm of $\mathbf{k}[n]$ never grows too large. Replacing $\Phi^{-1}[n]$ by $\Phi_{C L}^{-1}[n]$ in Eq. 2.21 completes the definition of the condition number-limited least squares (CNLLS) algorithm. Note that the source weight correlation matrix $\Phi[n]$ propagates with unlimited condition number. If the channel or desired mode changes abruptly, the transient response is faster if $\Phi[n]$ has not been condition number-limited. Appendix A contains a simple example demonstrating the importance of limiting the condition-number of the inverse and not $\Phi[n]$ itself.

Another consequence of an impersistent excitation is that the CNLLS estimate of the replica matrix may converge to an inaccurate estimate. From Eqs. 2.19-2.22, it is apparent that any value of $\hat{\mathbf{Q}}[n]$ yielding $\mathbf{a}[n]=0$ is a fixed point of the estimator. Writing $\mathbf{p}[n]=\mathbf{Q}[n] \mathbf{w}[n]$ reveals that $\mathbf{a}[n]=0$ is equivalent to $(\mathbf{Q}[n]-\hat{\mathbf{Q}}[n]) \mathbf{w}[n]=\mathbf{0}$ or $(\Delta \hat{\mathbf{Q}}[n]) \mathbf{w}[n]=\mathbf{0}$ where $\Delta \hat{\mathbf{Q}}[n]$ is the error in the current replica matrix estimate. 
This condition does not imply $\Delta \hat{\mathbf{Q}}[n]=0$, but only that the error in each row of the estimate is orthogonal to $\mathbf{w}[n]$. Consequently, $(\mathbf{Q}[n]-\Delta \mathbf{Q}[n])^{\dagger} \mathbf{p}_{d}$ does not necessarily give the $\mathbf{w}[n]$ minimizing $\left\|\mathbf{p}[n]-\mathbf{p}_{d}\right\|$. As discussed in Sec. 2.1, these fixed points are unstable equilibria, and in practice the observation noise appears to prevent the algorithm from stalling at these incorrect channel replica estimates. The resulting jitter in $\mathrm{w}[n]$ keeps the input persistent enough to prevent any severe errors in $\hat{\mathbf{Q}}[n]$.

\subsection{Kalman Filter Estimator}

The rows of $\mathbf{Q}[n]$ can also be estimated as the state vectors for $N$ separate Kalman filters. In order to apply the Kalman filter to the problem, the rows of $\mathbf{Q}[n]$ are modeled as evolving as separate first-order Gauss-Markov processes [55]. The covariance of the Gaussian process driving the state equations must be observed, computed from oceanographic models, or assumed. If the rows of $\mathrm{Q}[n]$ propagate independently, the state space equations can be written as

$$
\begin{aligned}
\mathbf{q}_{i}[n+1] & =\alpha_{i} \mathbf{q}_{i}[n]+\mathbf{f}_{i}[n] \\
p_{i}[n] & =\mathbf{q}_{i}[n] \mathbf{w}[n]+n_{i}[n],
\end{aligned}
$$

where $\mathbf{f}_{i}[n]$ is the Gaussian random process driving the state update equations with covariance $\mathbf{P}_{\mathbf{f}_{i}}[n], p_{i}[n]$ is the pressure observed at the $i^{\text {th }}$ hydrophone of the feedback array, and $n_{i}[n]$ is the observation noise at that hydrophone, with variance $\sigma_{n_{i}}^{2}$. Note the assumption of independence is very conservative in this context, as the estimators do not attempt to exploit any correlation between the rows of $\mathbf{Q}$. This also prevents the estimators from suffering any deterioration in performance due to possible mismatch between the true values of the correlations and the values used by the estimator.

Equations 2.26 and 2.27 are identical to the formulations of the state-space model 
in [43] and [55], except the format has been appropriately modified to make $\mathbf{q}_{i}[n]$ a row vector instead of a column vector. Exploiting the development of [43], the Kalman filter estimate of $\hat{\mathbf{q}}_{i}[n]$ can be written as

Prediction Step

$$
\begin{aligned}
\hat{\mathbf{q}}_{i}[n \mid n-1] & =\alpha_{i} \hat{\mathbf{q}}_{i}[n-1 \mid n-1] \\
\mathbf{P}[n \mid n-1] & =\alpha_{i}^{2} \mathbf{P}[n-1 \mid n-1]+\mathbf{P}_{\mathbf{f}_{i}}
\end{aligned}
$$

\section{Update Step}

$$
\begin{aligned}
\hat{\mathbf{q}}_{i}[n \mid n] & =\hat{\mathbf{q}}_{i}[n \mid n-1]+\frac{\left(p_{i}[n]-\hat{\mathbf{q}}_{i}[n \mid n-1] \mathbf{w}[n]\right) \mathbf{w}^{\mathrm{H}}[n] \mathbf{P}[n \mid n-1]}{\mathbf{w}^{\mathrm{H}}[n] \mathbf{P}[n \mid n-1] \mathbf{w}[n]+\sigma_{n_{i}}^{2}}, \\
\mathbf{P}[n \mid n] & =\mathbf{P}[n \mid n-1]-\frac{\mathbf{P}[n \mid n-1] \mathbf{w}[n] \mathbf{w}^{\mathrm{H}}[n] \mathbf{P}[n \mid n-1]}{\mathbf{w}^{\mathrm{H}}[n] \mathbf{P}[n \mid n-1] \mathbf{w}[n]+\sigma_{n_{i}}^{2}} .
\end{aligned}
$$

where $\hat{\mathbf{q}}_{i}[n \mid n]$ and $\hat{\mathbf{q}}_{i}[n \mid n-1]$ are $E\left\{\mathbf{q}[n] \mid p_{i}[0], \ldots, p_{i}[n]\right\}$ and $E\left\{\mathbf{q}[n] \mid p_{i}[0], \ldots, p_{i}[n-1]\right\}$ respectively. The covariance matrices are defined as

$$
\begin{aligned}
\mathbf{P}[n \mid n] & =\operatorname{cov}\{\mathbf{q}[n]-\hat{\mathbf{q}}[n \mid n]\} \\
\mathbf{P}[n \mid n-1] & =\operatorname{cov}\{\mathbf{q}[n]-\hat{\mathbf{q}}[n \mid n-1]\} .
\end{aligned}
$$

If $\mathbf{P}_{\mathbf{f}_{i}}$ and $\alpha_{i}$ are assumed to be identical for all the rows of $\mathbf{Q}$, and the observation noise $\mathbf{n}[n]$ is spatially white and Gaussian with variance $\sigma_{n}^{2}$, the row estimates may all be computed simultaneously as

Prediction Step

$$
\begin{aligned}
\hat{\mathbf{Q}}[n \mid n-1] & =\alpha \hat{\mathbf{Q}}[n-1 \mid n-1] \\
\mathbf{P}[n \mid n-1] & =\alpha^{2} \mathbf{P}[n-1 \mid n-1]+\mathbf{P}_{\mathbf{f}}
\end{aligned}
$$




\section{Update Step}

$$
\begin{aligned}
& \hat{\mathbf{Q}}[n \mid n]=\hat{\mathbf{Q}}[n \mid n-1]+\frac{(\mathbf{p}[n]-\hat{\mathbf{Q}}[n \mid n-1] \mathbf{w}[n]) \mathbf{w}^{\mathrm{H}}[n] \mathbf{P}[n \mid n-1]}{\mathbf{w}^{\mathrm{H}}[n] \mathbf{P}[n \mid n-1] \mathbf{w}[n]+\sigma_{n}^{2}} \\
& \mathbf{P}[n \mid n]=\mathbf{P}[n \mid n-1]-\frac{\mathbf{P}[n \mid n-1] \mathbf{w}[n] \mathbf{w}^{\mathrm{H}}[n] \mathbf{P}[n \mid n-1]}{\mathbf{w}^{\mathrm{H}}[n] \mathbf{P}[n \mid n-1] \mathbf{w}[n]+\sigma_{n}^{2}} .
\end{aligned}
$$

The Kalman filter algorithm begins with initial estimates $\hat{\mathbf{Q}}[0 \mid 0]$ and $\mathbf{P}[0 \mid 0]$. These may be obtained as specified for the CNLLS algorithm by sequentially activating each element of the source array, or estimated from a priori knowledge of the environment.

The Kalman filter estimator has an advantage over the CNLLS algorithm because the former provides mechanisms for incorporating and exploiting any additional information available about the spatial correlation of the noise or replica matrix evolution over time. For instance, if the observation noise is dominated by sea surface noise matching the Kuperman-Ingenito model, the $\sigma_{n_{i}}^{2}$ terms can be set appropriately for each row. Currently, no detailed and experimentally-verified models exist describing how the statistics of replica matrices are affected by oceanographic processes such as internal waves and tidal mixing. If experiments using single mode propagation and other techniques yield such models, this information can be incorporated into the estimator in two ways. First, these models could give predictions for $\mathbf{P}_{\mathbf{f}_{i}}$. Second, in many conditions such as internal wave propagation through the control feedback volume, the updating of the $\mathbf{q}_{i}$ 's will not be independent. An accurate model of internal wave dynamics could estimate the correlation between the rows of $\mathrm{Q}[n]$. If the rows are combined into one large $1 \times N L$ state vector, these correlation estimates would give $\mathbf{P}_{\mathbf{f}}$ for this new state vector, and the Kalman filter would exploit the correlation structure of these rows in updating its estimate of $\mathbf{Q}[n]$. The Kalman filter's ability to assimilate such information as it becomes available recommends it over the least squares approach. As noted earlier, the possible benefits of including such information in the model comes with the risk of concomitant mismatch problems if the information is incorrect. 
Two recent papers have presented preliminary results on the statistics and dynamics of interval wave processes. Lynch et al. [56] measured travel-time variation in acoustic propagation due to large scale internal tides in the Barrents Sea Frontal Region. Unfortunately, equipment failure prevented the determination of directional spectra of the internal wave field, and also scuttled plans to observe propagation along a path which would have isolated internal wave effects on acoustics from the effects of frontal dynamics and bottom layer fluid dynamics. Candy and Chambers proposed a discrete-time state-space model for internal wave dynamics [57]. They used this model to enhance the signature of internal waves observed by a horizontal current meter array. The state space model required careful hand-tuning of the covariance matrices for the specific environment and waves in order to detect the internal waves. As more data is obtained about internal wave dynamics, a careful evaluation of the potential benefits versus risks must be made before incorporating the model statistics into the Kalman filter.

The Kalman filter estimator suffers when the input sequence is not persistent. The input sequence $\mathbf{w}[n]$ plays the role of the output matrix $\mathbf{C}[n]$ in Eq. 1.34. A system is defined to be uniformly completely observable if there exist $\alpha$ and $m$ such that the smallest eigenvalue of the observability gramian $\mathbf{M}[n, n+m]$ is greater than $\alpha$ for all $n$ [43]. The Kalman filter to estimate the state of a system in the form of Eqs. 1.33 and 1.34 will have exponentially stable dynamics if the pair of matrices $\left(\mathbf{A}[n], K_{\mathbf{n n}}^{-1 / 2}[n] \mathbf{C}[n]\right)$ are uniformly completely observable, and the pair $\left(\mathbf{A}[n], \mathbf{K}_{\mathbf{f f}}[n]\right)$ are uniformly completely observable. The former criteria is the critical one for the Kalman filter replica matrix estimator. For the state space model described in Eqs. 2.26 and 2.27, the observability criteria applies to $\left(\alpha_{i} \mathbf{I}, \sigma_{n_{i}}^{-1} \mathbf{w}[n]\right)$, giving an observability gramian

$$
\mathbf{M}[n, n+m]=\sigma_{n_{i}}^{-2} \sum_{\ell=n}^{n+m} \alpha_{i}^{2(n+m-\ell)} \mathbf{w}[\ell] \mathbf{w}^{\mathrm{H}}[\ell] .
$$


If $\mathbf{w}[n]$ converges to a fixed value, this matrix will not satisfy the condition for uniform observability. If $\left|\alpha_{i}\right|<1$, it will only exacerbate this conditioning problem since $\mathbf{w}[n]$ 's earlier excursions away from the final value will be weighted even less. The control algorithm's tendency to produce an impersistent input sequence means the Kalman filter cannot theoretically be guaranteed to converge. However, the algorithm had no difficulty converging in either simulations or laboratory waveguide tests.

\subsection{Least Mean Squares Estimator}

The Least Mean Square (LMS) Algorithm [13], [54] is a computationally simple and commonly used algorithm. In terms of the variables of the replica matrix estimation problem, the algorithm adaptively chooses a row vector $\hat{\mathbf{q}}[n]$ to give the best least squares solution to

$$
p_{i}[n]=\hat{\mathbf{q}}_{i}[n] \mathbf{w}[n] .
$$

The LMS algorithm approximates the steepest descent algorithm by using the instantaneous values of $p_{i}[n]$ and $\mathbf{w}[n]$ to estimate the cross-correlation vector $E\left\{p_{i}[n] \mathbf{w}^{\mathrm{H}}[n]\right\}$ and autocorrelation matrix $E\left\{\mathbf{w}[n] \mathbf{w}^{\mathrm{H}}[n]\right\}$. The resulting estimator is

$$
\hat{\mathbf{q}}_{i}[n+1]=\hat{\mathbf{q}}_{i}[n]+\mu\left(p_{i}[n]-\hat{\mathbf{q}}_{i}[n] \mathbf{w}[n]\right) \mathbf{w}^{\mathrm{H}}[n],
$$

where $\mu$ is a scalar controlling the correction step size at each iteration. If the input vectors $\mathbf{w}[n]$ are independent and persistent, and $\mathbf{w}[n]$ and $p_{i}[n]$ are mutually independent Gaussian random variables independent of all earlier values of $p_{i}[n]$, the LMS algorithm can be shown to converge in the mean to $\mathrm{q}_{i 0}$, the Wiener estimate of $\mathbf{q}_{i}[n]$ as $n \rightarrow \infty$. Even when these stringent conditions are not satisfied, the LMS algorithm is often still effective and used in practice because of its computational simplicity compared to algorithms such as those described in the previous two sec- 
tions. One problem with the LMS algorithm given by Eq. 2.40 is the norm of the correction $\mu\left(p_{i}[n]-\hat{\mathbf{q}}_{i}[n] \mathbf{w}[n]\right) \mathbf{w}^{\mathrm{H}}[n]$ depends on the norm of the input vector $\mathbf{w}[n]$. This can result in a convergence rate that depends on the input sequence. To reduce this dependence, the normalized LMS [54] algorithm uses $\mu=\tilde{\mu} /\left(c+\|\mathbf{w}[n]\|^{2}\right)$. The factor of $\|\mathbf{w}[n]\|^{2}$ normalizes the step size by the norm of the input vector, reducing the dependence of the convergence rate on the input sequence. The constant $c$ prevents $\mu$ from growing too large if $\|\mathrm{w}[n]\|^{2}$ grows very small.

The normalized LMS algorithm can be used to estimate all the rows of $\hat{\mathbf{Q}}[n]$ simultaneously by

$$
\hat{\mathbf{Q}}[n+1]=\hat{\mathbf{Q}}[n]+\frac{\tilde{\mu}}{c+\|\mathbf{w}[n]\|^{2}}(\mathbf{p}[n]-\hat{\mathbf{Q}}[n] \mathbf{w}[n]) \mathbf{w}^{\mathrm{H}}[n] .
$$

This equation reveals the normalized LMS estimator has the same fixed point as the CNLLS estimator. When the estimator errors for the rows of $\hat{\mathbf{Q}}[n]$ are orthogonal to the source array vector $\mathbf{w}[n]$ the LMS estimator will have same convergence problems caused by an impersistent input sequence as the CNLLS estimator. Comparing Eqs. 2.41 and 2.19-2.22 indicates the LMS estimator is equivalent to the CNLLS estimator with $\Phi[n]$ held fixed at $\mu \mathbf{I}$. Thus, the LMS estimator is not using any information about the past values of $\mathbf{w}[n]$ to update $\hat{\mathbf{Q}}[n]$. The CNLLS estimator will generally converge and respond to transients better because of its use of this information. If the weight sequence $\mathrm{w}[n]$ is distributed such that the $E\{\boldsymbol{\Phi}\}=\mathbf{I}$, the CNLLS and LMS estimators will be equivalent in the mean sense. However, the constraint of exciting a fixed pressure field will generally require $\mathbf{w}[n]$ to remain fixed or nearly fixed once the estimate of $\hat{\mathbf{Q}}[n]$ has converged close to the true $\mathbf{Q}$, and thus generally $E\{\Phi\} \neq \mathrm{I}$. In this situation, the LMS estimator is not expected to perform as well as the CNLLS estimator. The experiments presented in Chapter 4 verify this. 


\section{Chapter 3}

\section{Simulation Results}

This chapter presents the results of using the control algorithm presented in the last chapter in several simulated ocean environments. All of the environments were simulated with the finite-element parabolic equation (FEPE) model for acoustic propagation [21]. This is an especially good model to use for these simulations because it is not a mode-based propagation model. Consequently, this eliminates any concerns about the propagation model generating a single mode because it assumes the propagation takes place in discrete modes. The shallow water environments are based on observed sound speed profiles from the continental shelf in the region of $41^{\circ}$ $\mathrm{N} 71^{\circ} \mathrm{W}[58]$. Figure 3-1 indicates the specific transect where the profiles used in the experiments were measured. Section 3.1 confirms the algorithm works for a simple range- and time-invariant environment. Sections 3.2 and 3.3 simulate two common shallow water bathymetric features: a rock outcropping and a downsloping wedge. Both of these features affect acoustic propagation and would cause problems for an open loop control algorithm which assumed the modes propagated adiabatically downrange. The final section, Section 3.4, simulates the propagation of a solitary internal wave through the feedback volume over the course of roughly twenty-five minutes. The successful performance of the feedback control algorithm in all these environments offers encouragement for its successful deployment in an oceanographic 
experiment.

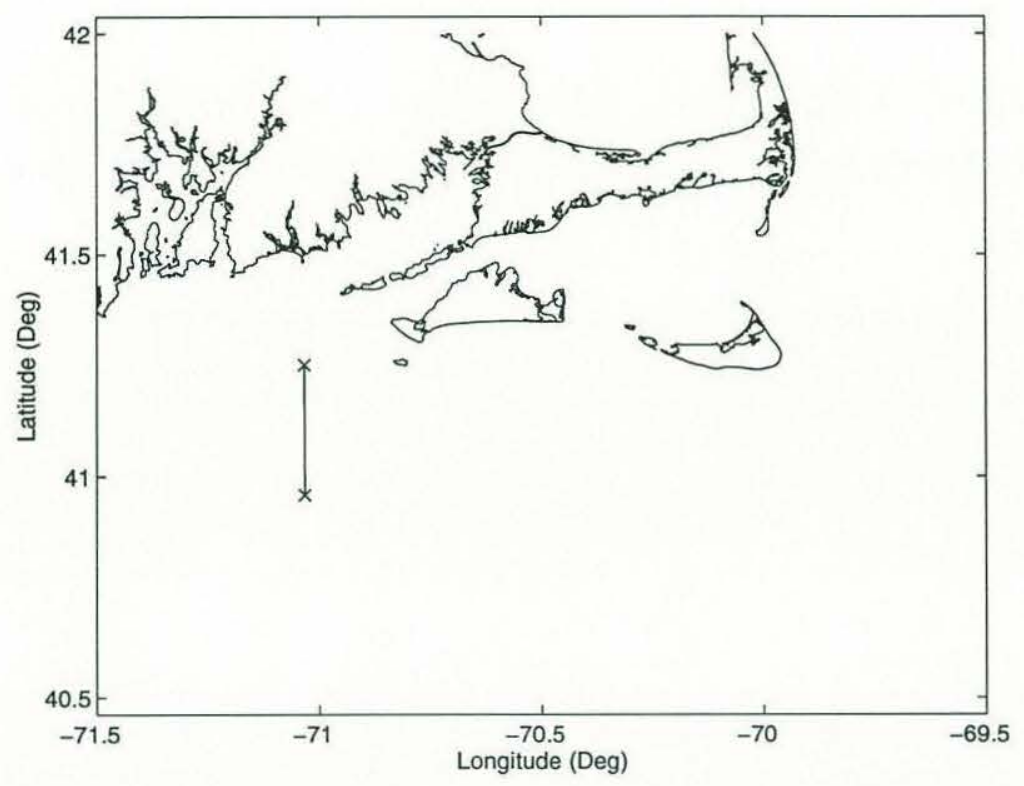

Figure 3-1: Hydrographic Transect Providing Environmental Data for Simulations

\subsection{Range-Invariant Environment}

The simulations in this section verify that the feedback control algorithm works for a simple but realistic shallow water environment. In addition, the performance of the algorithm is evaluated over a range of estimator parameters. For the CNLLS estimator, the parameter was the exponential forgetting factor, while the Kalman filter trials varied the autoregressive parameter $\alpha$ and the innovation covariance $\mathbf{P}_{\mathbf{f}}$ used by the Kalman filter's state space model for the replica matrix dynamics in Eqs. 2.342.37. The environment used was time-invariant and homogeneous in range with the vertical profile shown in Figure 3-2. The experiments were all configured with the source and feedback arrays separated by $1 \mathrm{~km}$, and the propagation frequency was $400 \mathrm{~Hz}$. At this frequency, the environment supports nine trapped modes. All of the evanescent modes and continuum energy will be completely attenuated over the $\mathrm{km}$ distance between the source and feedback arrays [18]. The source array consists of 
10 omni-directional point sources, and the feedback array of 19 hydrophones. The replica matrix at this frequency for the environment was computed using a version of FEPE modified to give complex replica vectors rather than transmission loss.

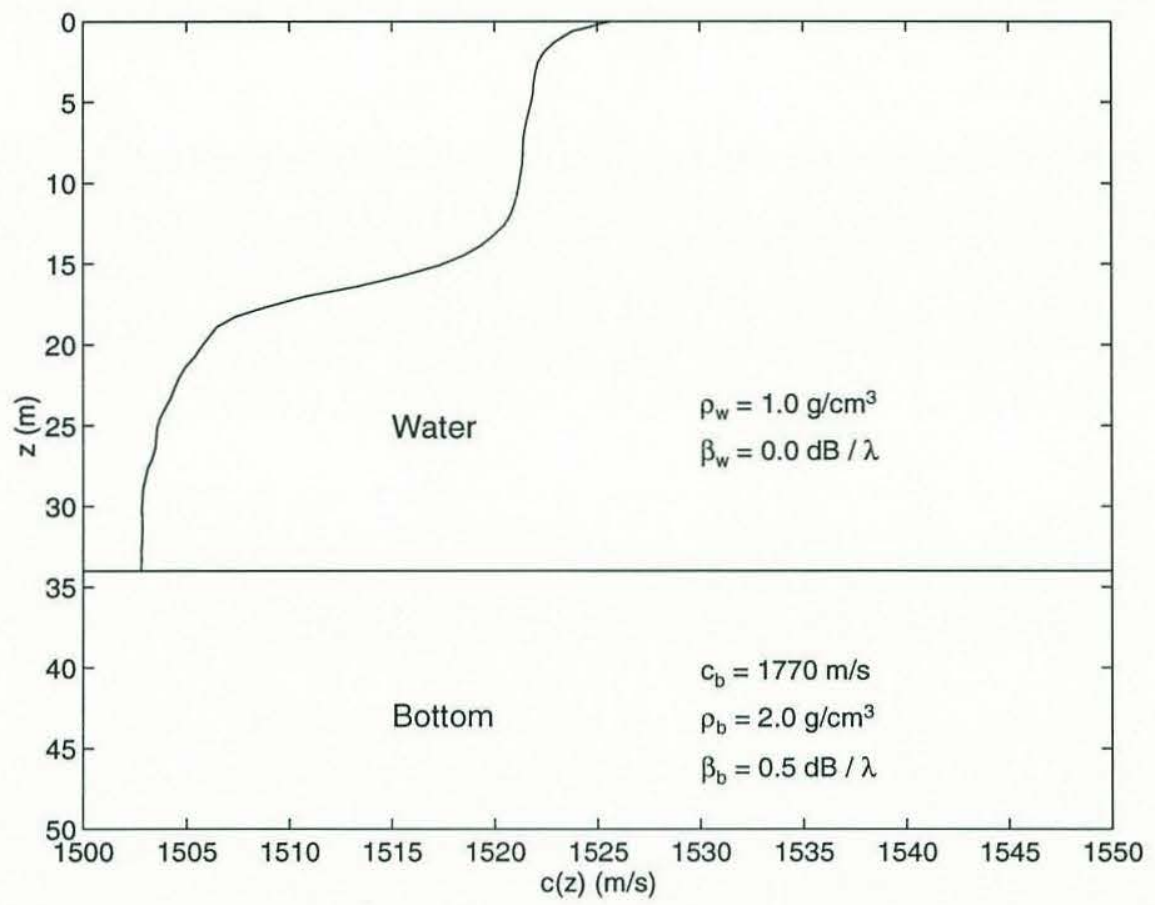

Figure 3-2: Vertical Profile of Range-Invariant Environment

Figure 3-3 plots the mode shapes for the nine trapped acoustic modes of this environment as computed by FEMODE, a finite-difference mode computation program. Figure 3-3 also displays the shape of the sound speed profile at the left edge of the plot for purpose of comparison with the mode shapes. The feedback array samples the mode shapes well, and so $\Psi_{R}$ has a condition number of roughly 1.25. Because $\Psi_{R}$ is well-conditioned, choosing the source array weights to minimize the error between the desired and obtained profiles in the pressure domain will give a high fidelity single mode.

The experiments each consisted of 100 independent trials attempting to excite mode two. Each trial started from a random $\hat{\mathbf{Q}}[0]$ and then allowed the algorithm to converge for 250 iterations. The observation noise at the feedback array was 

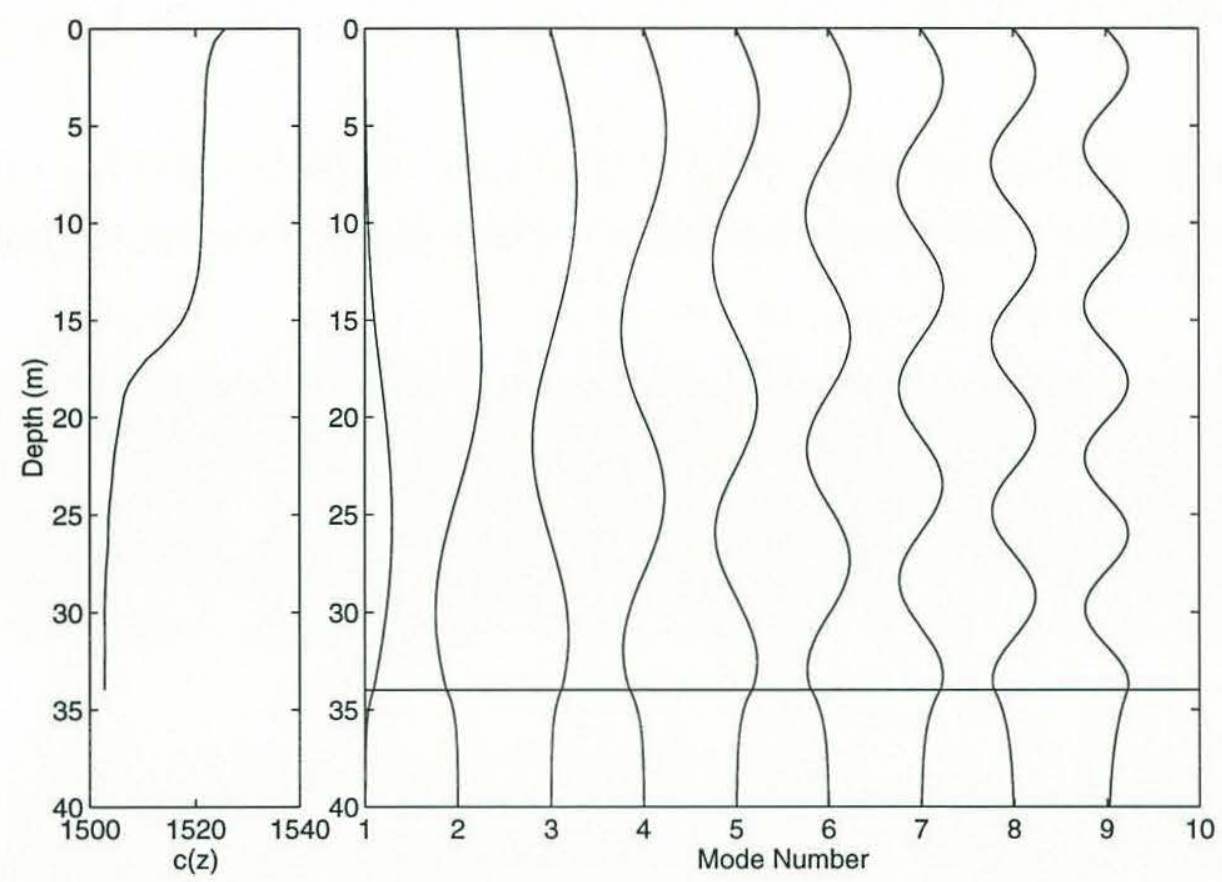

Figure 3-3: Mode Shapes for Range-Invariant Environment

spatially white, and $20 \mathrm{~dB}$ below the energy in the desired pressure profile for mode two. This SNR is relatively high but realistic given the short distance between the source and feedback arrays. For the scenario shown in Fig. 1-1, the excited field must be strong at the feedback array if it is to be measurable at the observation array, which is much further downrange. If the pressure field at the feedback array is not significantly above the observation noise, the observation array may have difficulty separating the signal due to the mode input to the observation volume from noise.

Figure 3-4 shows the convergence of the control algorithm using the CNLLS estimator for $\mathbf{Q}$. The figure plots the mean performance over 100 trials for three different forgetting factors, as well as the least squares bound obtainable from perfect knowledge of $\mathbf{Q}$. The three forgetting factors correspond to effective window lengths of 50, 100 and 200 iterations, where the effective window length $N_{\gamma}$ for a forgetting 
factor $\gamma$ is defined to be the number of iterations required for it to decay to 0.1 , i.e.,

$$
\gamma^{N_{\gamma}}=0.1
$$

The curves in Figure 3-4 plot the mean signal-to-error ratios (SERs) averaged over all trials for each experiment. The SER is defined to be the ratio of the energy in the desired pressure profile to the energy in the error at each iteration, i.e.,

$$
10 \log _{10}\left(\frac{\left\|\mathbf{p}_{d}\right\|^{2}}{\left\|\mathbf{p}_{d}-\mathbf{p}[n]\right\|^{2}}\right)
$$

where $\mathbf{p}[n]$ is the pressure observed at the feedback array for the current iteration including all observation noise. The error term $\left\|\mathbf{p}_{d}-\mathbf{p}[n]\right\|^{2}$ appears in the denominator of the SER expression, while the numerator $\left\|\mathbf{p}_{d}\right\|^{2}$ is fixed for these experiments. Thus, decreasing the error between the desired and observed pressure vectors will increase the SER. The experiments indicate that the performance does not vary much with changes in forgetting factor. The "LS Bound" at $55 \mathrm{~dB}$ is the least-squares bound on the performance that could be attained with perfect knowledge of $\mathbf{Q}$ and no observation noise. This bound is finite because of numerical differences between the methods for computing the pressure profiles for mode two and the replica matrix Q. As a result, a small component of $\mathbf{p}_{d}$ is not in the span of $\mathbf{Q}$.

Because the observed pressure includes noise, there is a practical bound on the curves in Fig. 3-4 considerably below the LS bound. Even if the algorithm perfectly excited the desired pressure field, the observation noise would still limit the performance to $20 \mathrm{~dB}$. Figure 3-5 shows the results of several experiments using the CNLLS estimator with decreasing observation noise (increasing SNR). Again, each curve is the average performance over 100 trials of the same experiment. In each case, the SER approaches the SNR demonstrating the performance of the algorithm in this environment is limited by the level of the observation noise. Figure 3-6 shows the desired pressure profile at the feedback array against the pressure obtained by a 
typical trial of this experiment with effective window length 100 . While the pressure obtained is not a perfect fit to the desired profile, it is a good approximation.

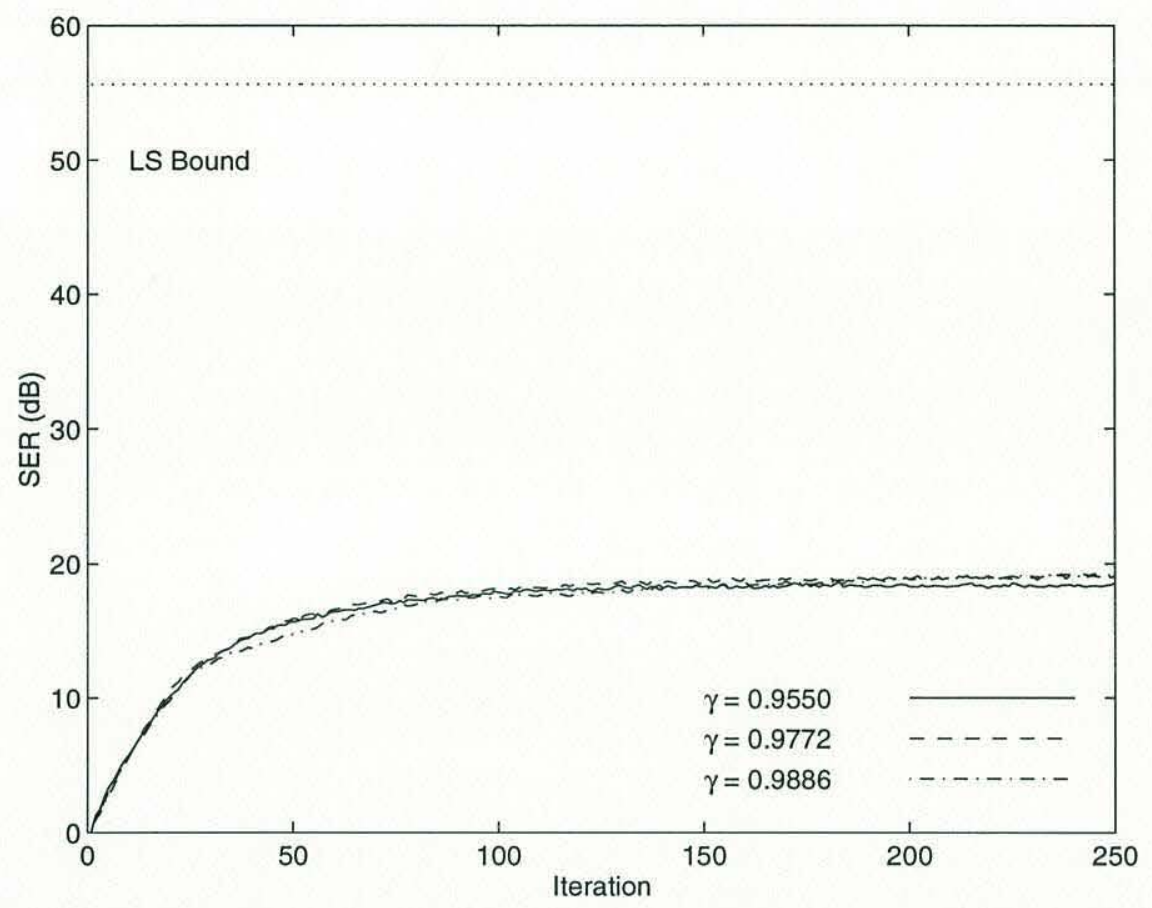

Figure 3-4: Ratio of Energy in Desired Profile to Energy in Mean Error for CNLLS Estimator

Figures 3-7 through 3-9 show the results of several simulations using the Kalman filter channel estimator with different values for $\alpha$ and $\mathbf{P}_{\mathbf{f}}$ in the Kalman filter's state space model for the dynamics of $\mathrm{Q}$. Note that this is only changing the parameters of the model used by the estimator, and does not change the properties of the real channel, which is time invariant. These experiments were performed with the same configuration, initialization and number of trials as the CNLLS experiments. For $\alpha=0.98$ and 0.99 , the experiments indicate the control algorithm performed best when a relatively large innovation covariance $\mathbf{P}_{\mathrm{f}}$ was used in the Gauss-Markov model for the evolution of the rows of $\mathbf{Q}$ i.e., Eq. 2.26. Choosing $\mathbf{P}_{\mathbf{f}}=10^{-6} \mathbf{I}$ gave the best performance for these values of $\alpha$. The mean absolute value of the elements of the true $\mathbf{Q}$ is $1.2 \times 10^{-3}$, so the standard deviation of the innovation process for 


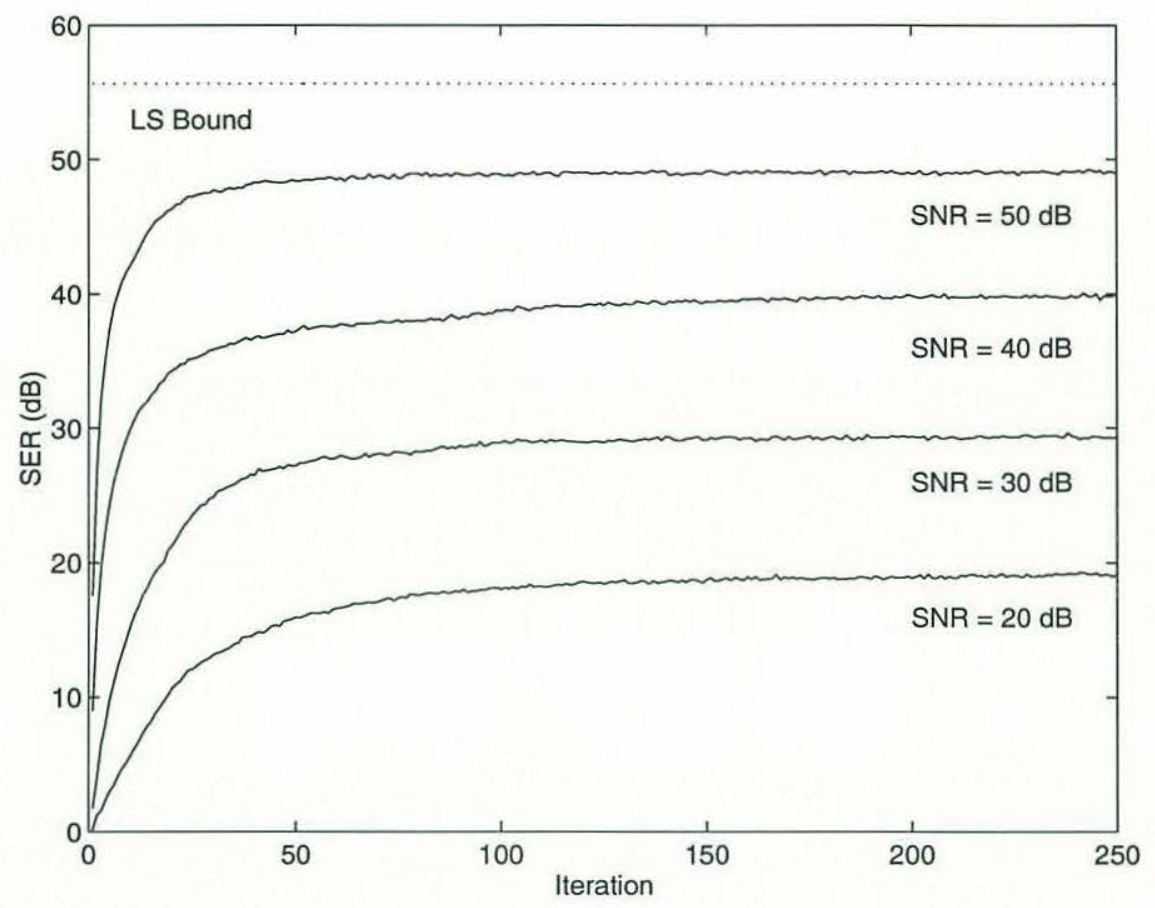

Figure 3-5: SER for CNLLS Estimator at different SNR levels

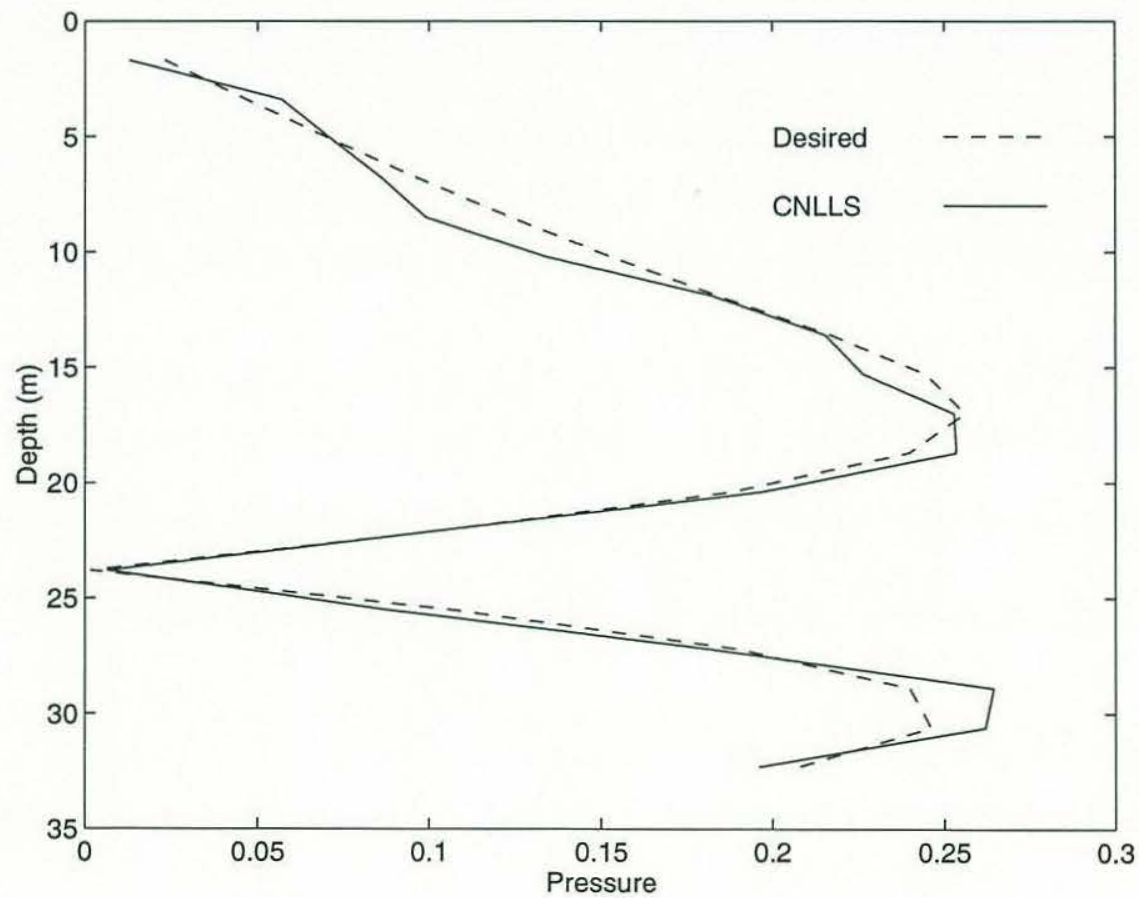

Figure 3-6: Vertical Profile of Pressure for Typical Trial of CNLLS Estimator 
these elements is on the same order as the mean replica matrix element. When $\alpha=1.0$, Figure 3-9 shows the estimator with $\mathbf{P}_{\mathbf{f}}=10^{-6} \mathbf{I}$ does noticeably worse than the others. Solving for the steady-state error covariance of the Kalman filter reveals that for $\alpha=1.0$ the error covariance of the Kalman filter is much larger for $\mathbf{P}_{\mathbf{f}}=10^{-6} \mathbf{I}$. It is sensible that a large value of $\mathbf{P}_{\mathbf{f}}$ would make the error variance grow even faster and cause worse performance than smaller values of $\mathbf{P}_{\mathbf{f}}$ when $\alpha=1.0$ so there is no decay. For all three values of $\alpha$ shown here, experiments using even larger values for $\mathbf{P}_{\mathbf{f}}$ caused worse performance. This is reasonable because a larger $\mathbf{P}_{\mathbf{f}}$ would mean the innovation portion of the Gauss-Markov process $\mathbf{f}$ would overwhelm the true values of the time-invariant $\mathbf{Q}$ the algorithm is attempting to estimate. Figure 3-10 plots the desired and obtained vertical pressure profiles for a typical trial with $\alpha=0.99$ and $\mathbf{P}_{\mathbf{f}}=10^{-6} \mathbf{I}$. Similar to the result shown in Figure 3-6, the profile obtained by the control algorithm is a close but not perfect fit to the desired profile.

Figure 3-11 plots the results of a simulation comparing the CNLLS with $\gamma=1$ to the Kalman filter with $\alpha=1$ and $\mathbf{P}_{\mathbf{f}}=0$. It can be demonstrated that for these parameter choices, the estimators are equivalent if the condition number is not limited in the CNLLS estimator. The curves in Fig. 3-11 are very close but not identical. Two factors may have caused this discrepancy. First, each curve is an average of 100 trials with the appropriate estimator. Each trial started with a different randomly chosen $\hat{\mathbf{Q}}[0]$. In the limit of an infinite set of trials, these averages should agree, but the sample averages for each estimator need not be identical after 100 trials. Second, the CNLLS estimator did limit the condition number of $\Phi^{-1}[n]$, so the estimators are not exactly equivalent when $\Phi$ becomes poorly conditioned. The increasing difference between the curves with increasing $n$ supports this explanation. If the condition number-limiting is a factor, it will only matter once $\mathbf{w}[n]$ has converged and $\Phi[n]$ becomes poorly-conditioned. This will take several iterations, and the effect will become more severe as $\Phi$ becomes more poorly conditioned over time. The curves in Fig. 3-11 start very close together and only diverge slowly as the 
would be expected as the conditioning of $\Phi$ grew worse. This supports the hypothesis the condition number-limiting is the primary factor responsible for the difference between the estimators. On the whole, the close agreement between the estimators in this experiment offers reassurance that the estimators are correctly implemented.

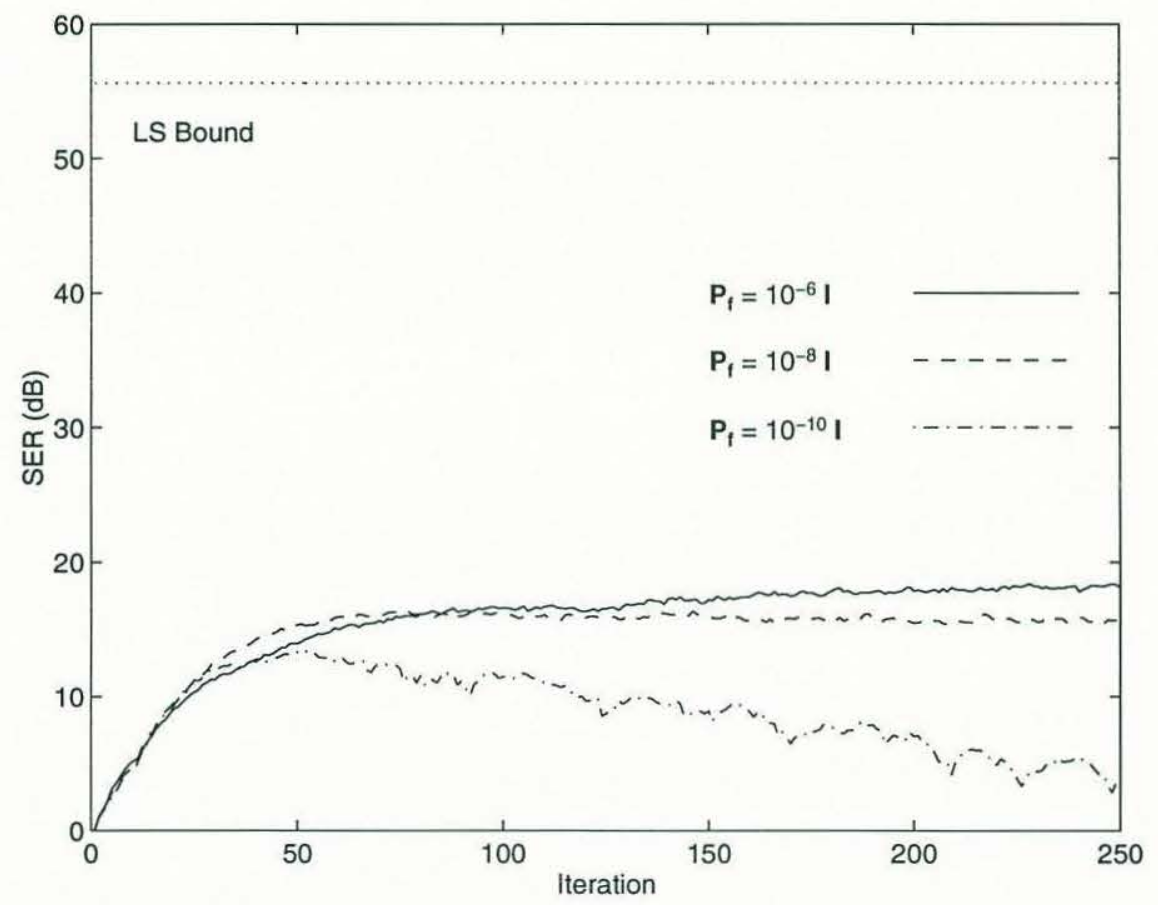

Figure 3-7: Ratio of Energy in Desired Profile to Energy in Mean Error for Kalman Filter Estimator with $\alpha=0.98$

\subsection{Rock Outcropping}

This section examines the performance of the control algorithm when the region between the source and feedback arrays includes a rock outcropping. Such features are common in shallow water environments, and the strong interaction of the pressure field with the bottom means these bathymetric features have an important effect on propagation. Given very accurate and detailed environmental measurements, an open loop control algorithm could theoretically still generate a single mode in the 


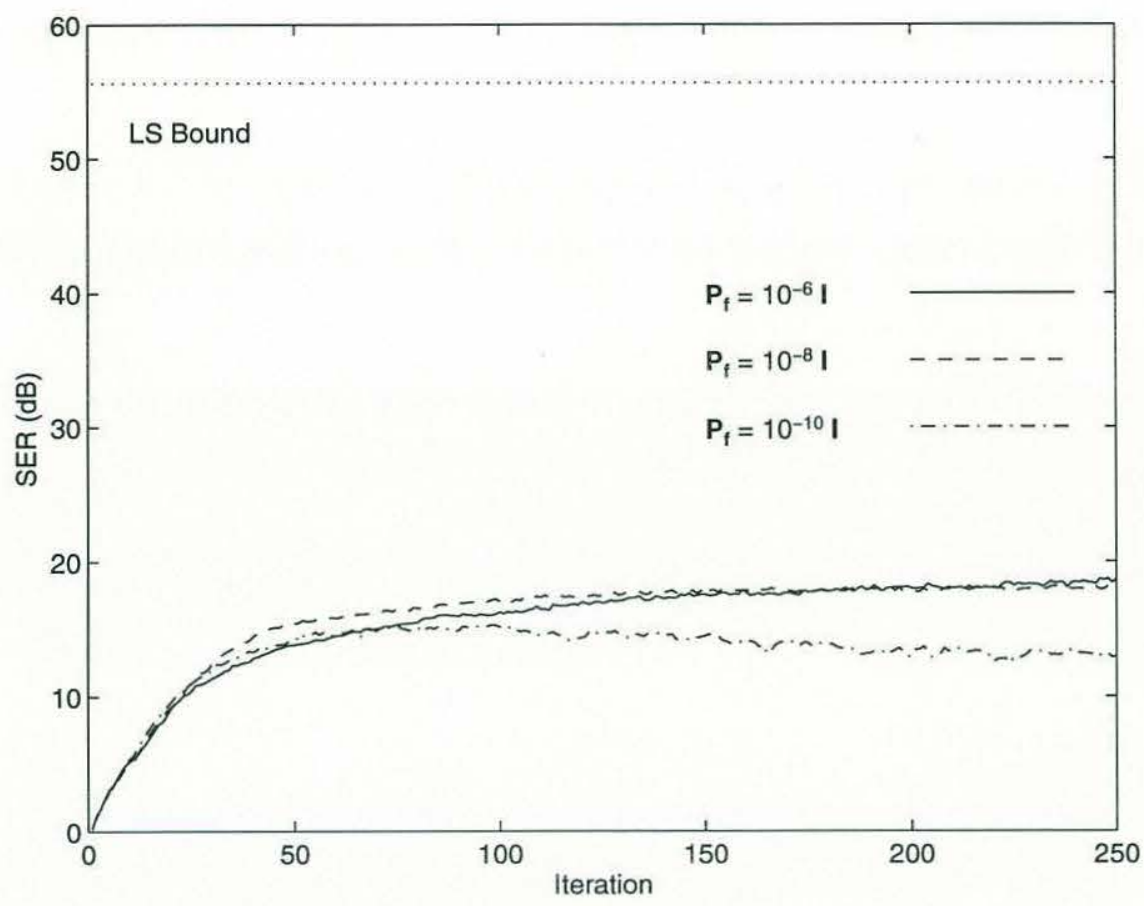

Figure 3-8: Ratio of Energy in Desired Profile to Energy in Mean Error for Kalman Filter Estimator with $\alpha=0.99$

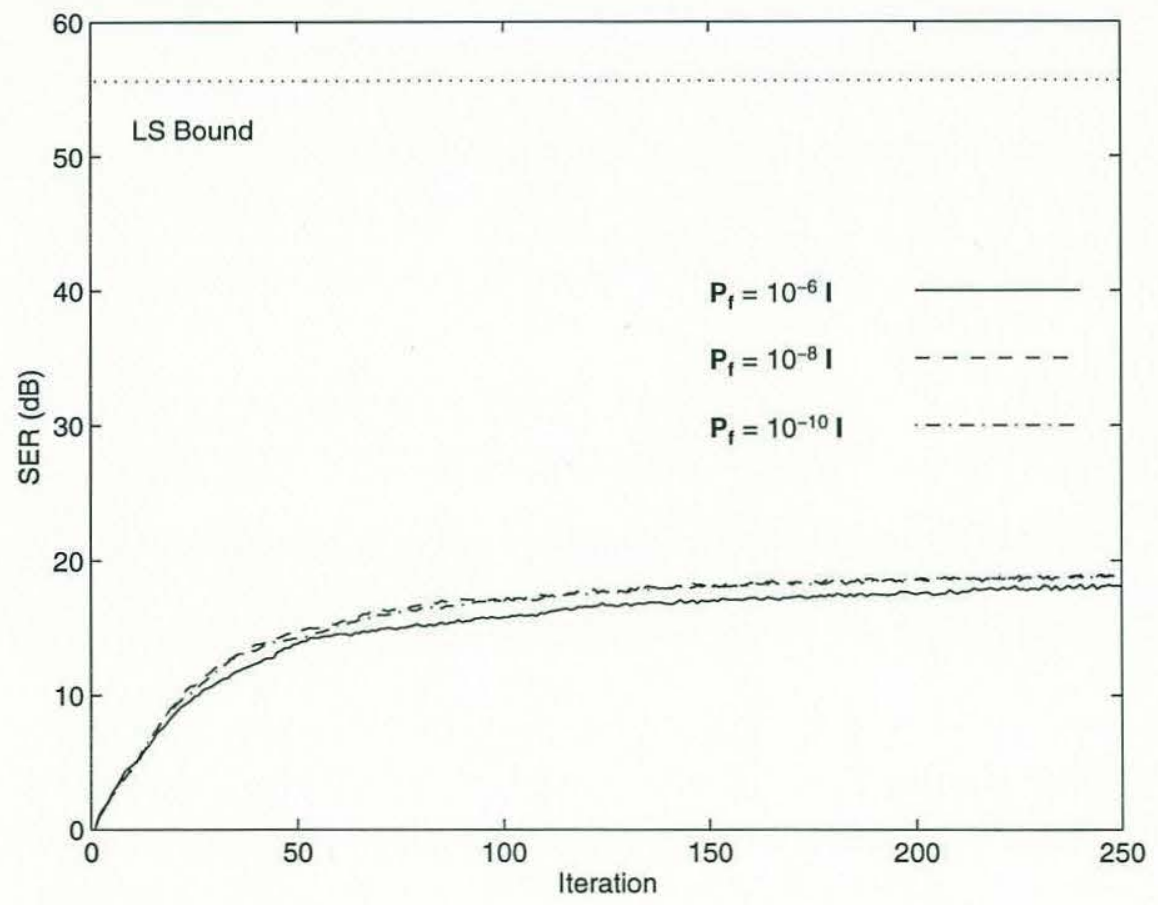

Figure 3-9: Ratio of Energy in Desired Profile to Energy in Mean Error for Kalman Filter Estimator with $\alpha=1.0$ 


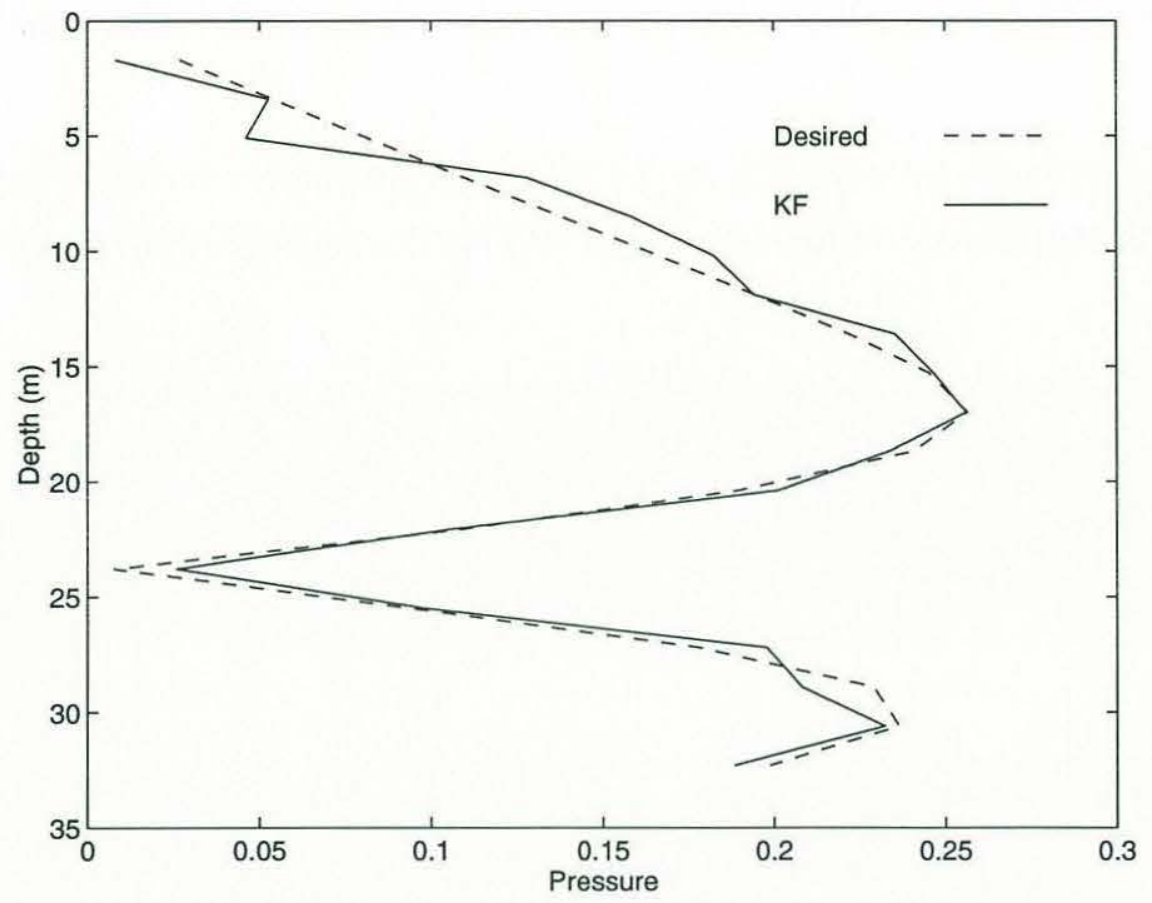

Figure 3-10: Vertical Profile of Pressure for Typical Trial of Kalman Filter Estimator with $\alpha=0.99, \mathbf{P}_{\mathbf{f}}=10^{-6} \mathbf{I}$.

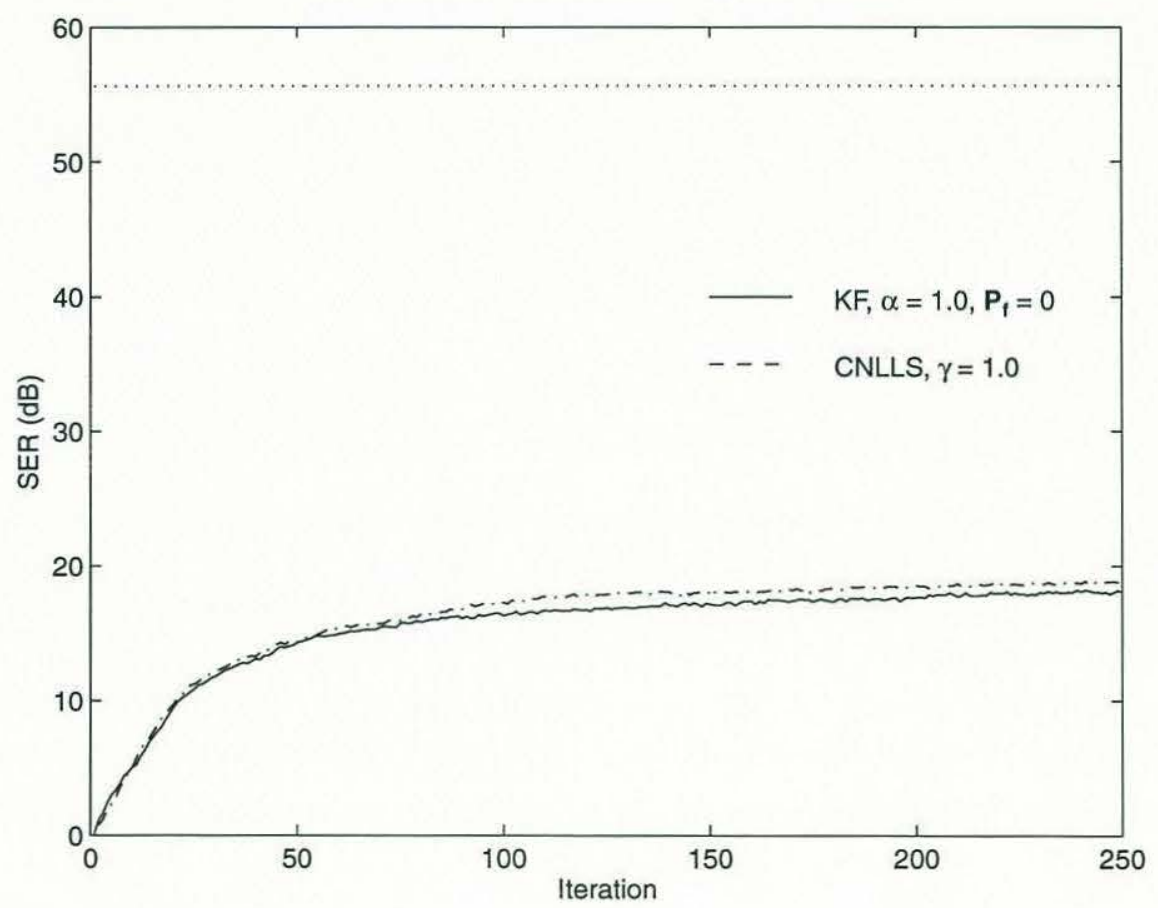

Figure 3-11: Comparison of CNLLS and Kalman filter estimators 
presence of such an outcropping. An echosounder survey could give a reasonably accurate bathymetric profile of the environment, though it would still have uncertainty in depth on the order of a quarter to half wavelength (1-2m). The acoustic effect of this uncertainty would probably be small, but not insignificant. Measurements of the geophysical parameters for the specific location would require exhaustive core and sediment sampling. However, representative measurements of these parameters exist for most coast regions, and generally these measurements would be acceptable approximations. The open loop algorithm would start with the available environmental data and try to compute the replica matrix from acoustic propagation models. The source array weights would then be set to be those the computed replica matrix predicted would give the desired pressure field at the start of the observation volume. In contrast, the feedback control algorithm estimates the replica matrix directly from acoustic observations, removing the intermediate step of computing the replica matrix from hydrographic and bathymetric observations. To whatever extent the geophysical parameters of the environment are relevant to the mode excitation experiment, they should manifest themselves in the observed pressure data.

Figure 3-12 shows the propagation environment with the outcropping. Other than the $6 \mathrm{~m}$ tall outcropping at $r=200 \mathrm{~m}$, this environment is identical to the range-invariant environment used in Section 3.1. The source array is at $r=0 \mathrm{~m}$ and the feedback array at $r=1000 \mathrm{~m}$. The outcropping has physical properties similar to those of limestone or basalt [32]. The experiments in this section will assume the extreme scenario that no a priori information was available about the rock outcropping. The open loop algorithm assumes it is operating in the range-invariant environment shown in Fig. 3-2. Generally, better information will be available, but this exaggerated mismatch demonstrates the attractive possibility of using the feedback array without any a priori knowledge of the environment.

The presence of this outcropping has a strong effect on the acoustic propagation. Figure 3-13 plots the performance of the feedback control algorithm using both 


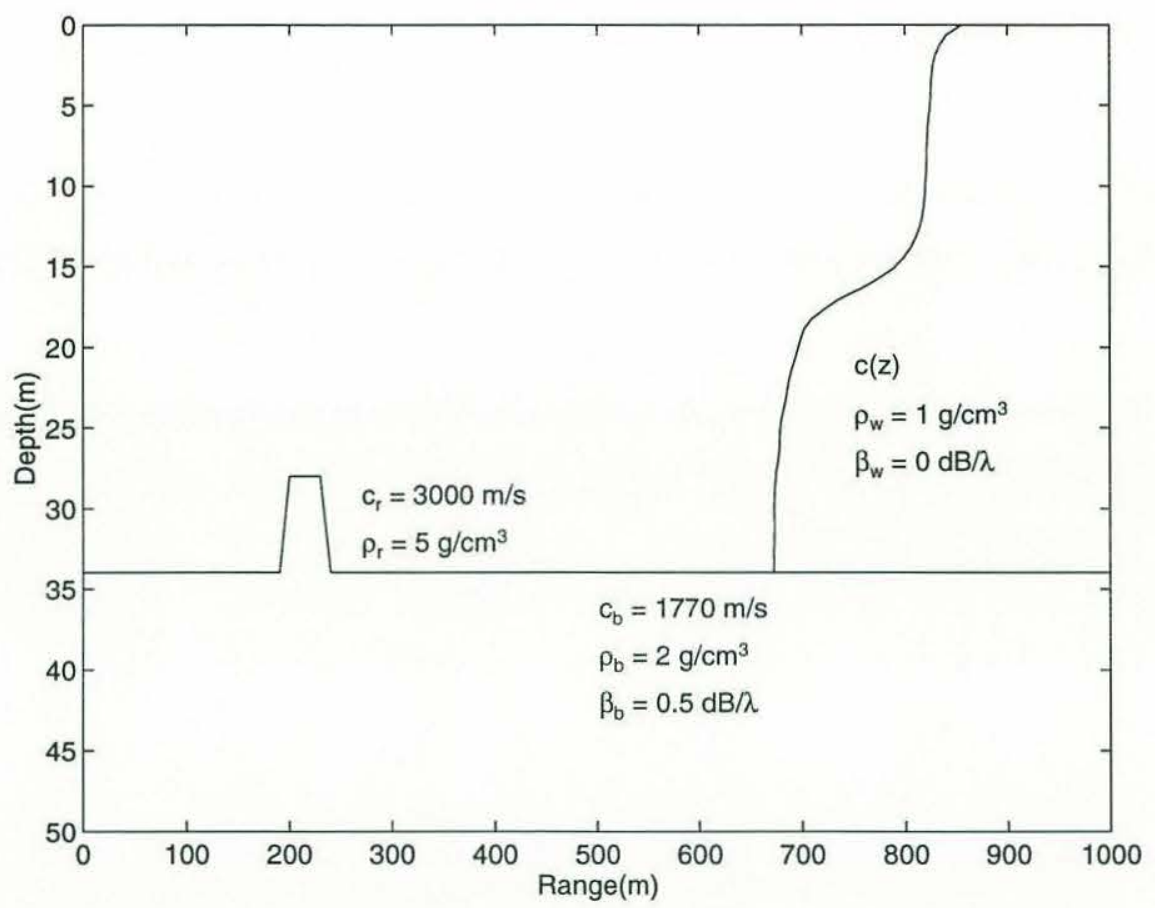

Figure 3-12: Rock Outcropping Environment

estimators, as well as for an open-loop controller which assumes the environment is homogeneous in range with the vertical profile observed at the source array. All the curves are the average over 100 trials initialized with random replica matrices. The CNLLS estimator used an effective window length of 100, while the Kalman filter had $\alpha=0.99$ and $\mathbf{P}_{\mathbf{f}}=10^{-6} \mathbf{I}$. The figure shows both estimators converge to allow the feedback controller to excite a single mode. However, the very poor performance of the open loop controller indicates the importance of accounting for the effect of the outcropping on propagation. By assuming the environment was range-invariant and that modes would propagate adiabatically, the open-loop controller generated a pressure profile in the far field which was a poor approximation to the desired pressure profile, and likely unacceptable as a source signal for oceanographic observations. Figure 3-14 shows vertical pressure profiles at the feedback array for one trial after the estimators had converged. From these plots, it is apparent that the feedback control algorithm gives a good approximation to the desired pressure profile using 
either estimator. The open-loop controller generates a profile that is unacceptable as a single mode source.

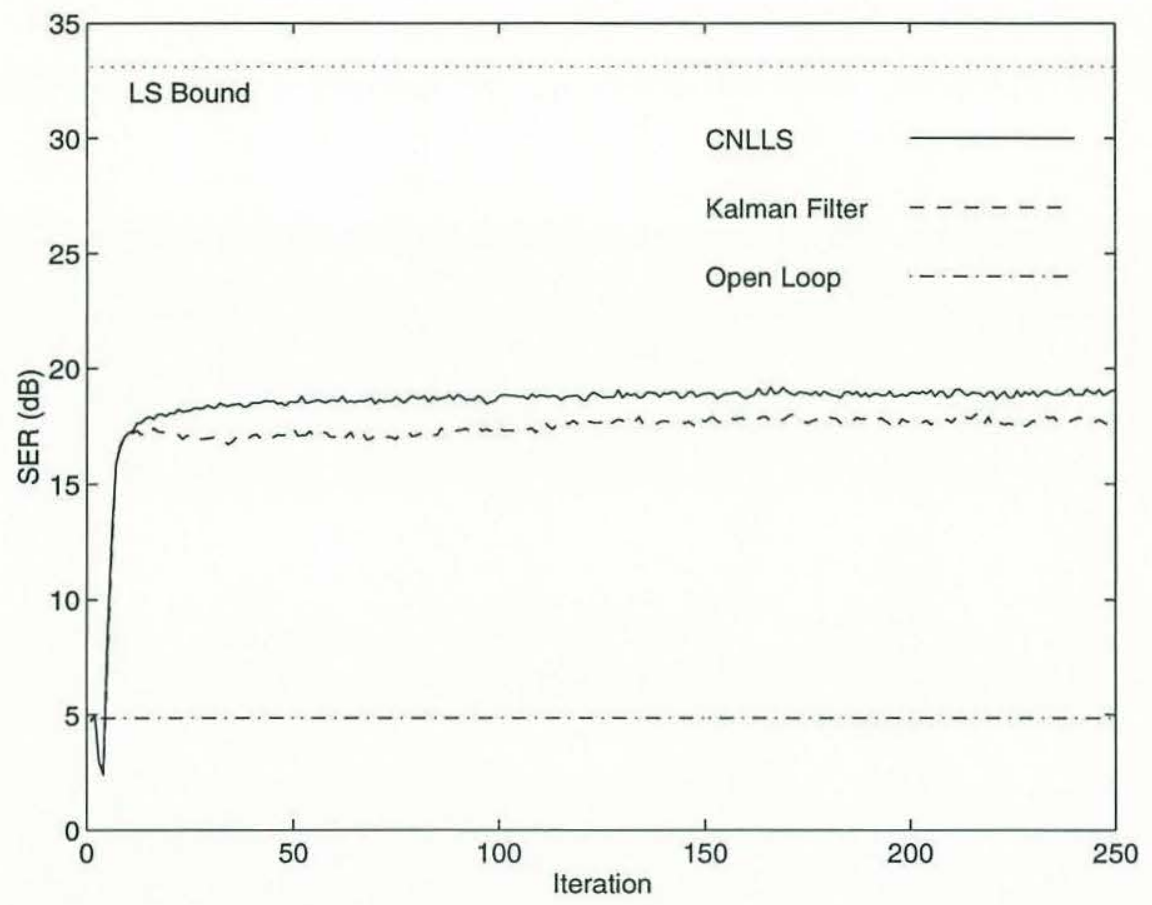

Figure 3-13: Performance of Estimators For Rock Outcropping Environment

Figures 3-15 and 3-16 present a revealing contrast between the pressure fields excited by the open loop and feedback control algorithms. Both figures plot acoustic intensity as a function of range and depth, where high intensity is indicated by a light shading. The intensity has been normalized to remove the effect of cylindrical spreading. The desired mode was mode two and the environment was the rock outcropping environment shown in Figure 3-12 in both cases. Figure 3-15 shows the pressure profile versus range and depth for the field excited by the open loop control algorithm, as well as the vertical profile of the magnitude of the desired pressure field for mode two on the left side of the figure for reference. Close to the source array, the pressure field can be seen to be settling towards mode two, with nulls developing at the surface and $z=24 \mathrm{~m}$. When the wave is incident upon the outcropping at $r=200 \mathrm{~m}$, the energy is scattered into several modes, generating the complicated 


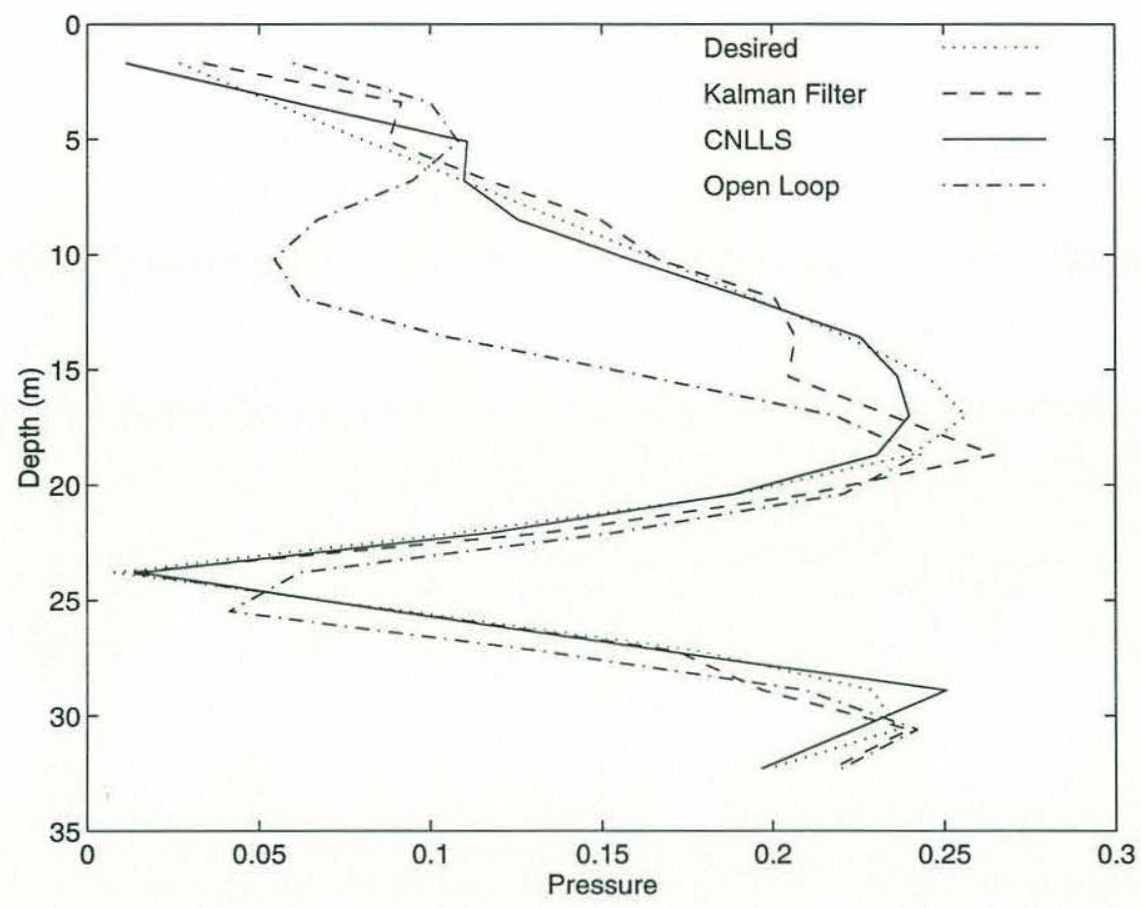

Figure 3-14: Vertical Profile of Pressure for a Typical Trial in Rock Outcropping Environment

interference pattern seen downrange of the outcropping. Based on this pattern, it is clear the pressure field entering the observation volume consists of several modes, and not the desired single mode.

Figure 3-16 plots the intensity for the feedback controller using the CNLLS estimator. For this algorithm, the source array initially excites a very complicated interference pattern containing a rich variety of modes. When this pressure field impinges upon the rock outcropping, the energy scatters such that all the undesired modes cancel out, leaving only mode two propagating. By the time the field reaches the feedback array at the start of the observation volume $(r=1000 \mathrm{~m})$, there is a well-developed pressure null at a depth of $z=24 \mathrm{~m}$. This corresponds well with the desired vertical pressure profile shown at the left side of the figure. The feedback control algorithm successfully estimates and compensates for the scattering effect of the rock outcropping to produce the desired pressure field in the far field. 
These results can be interpreted in terms of the matched signal paradigm discussed in Section 1.5. The open loop algorithm assumes an adiabatic environment and transmits the signal desired at the feedback array immediately from the source array. The outcropping distorts the transmitted mode two, coupling the energy into several modes. The feedback control algorithm estimates the environmental effect on the propagation, then transmits a complicated waveform which is matched to the environment so the pressure field at the start of the observation volume consists solely of mode two. This is analogous to the work done by Parvulescu and Clay [46], [45] only here the desired waveform is concentrated in the mode domain rather the time domain. The feedback control algorithm transmits a waveform that is initially not concentrated in the mode domain such that the pressure field at the start of the observation volume contains a single mode. The open loop controller does not account for any channel effects, and the field it generates at the start of the observation volume is not concentrated in the mode domain, but has energy in several modes.

\subsection{Downsloping Wedge}

A downsloping wedge is a common feature in many shallow water environments. This section presents the results of simulating the deployment of the feedback control algorithm in a $2^{\circ}$ downsloping wedge using the profiles observed in [58]. For the portions of the wedge deeper than the bottom used in the previous section, the deepest measurement of sound speed from the previous section is extended downward to form a isovelocity layer above the bottom in the wedge. The experiments described in this section will compare the performance of the feedback control algorithm to the open-loop algorithm, which assumes the modes excited at the source array propagate downslope without coupling. In reality, the slope causes coupling among the modes as they propagate downrange so the field excited by the open-loop algorithm 

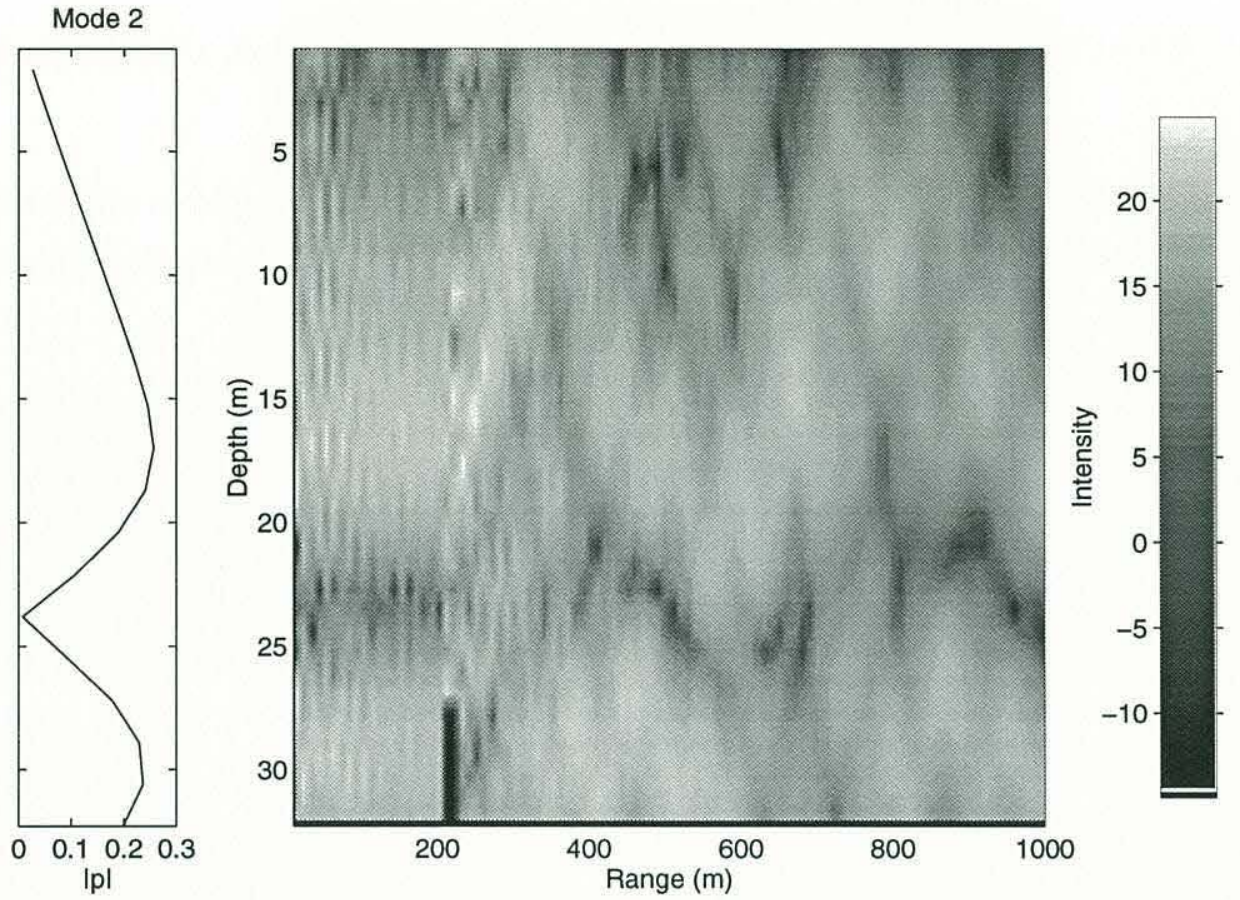

Figure 3-15: Pressure Field Excited by Open Loop Controller in Rock Outcropping Environment

Mode 2
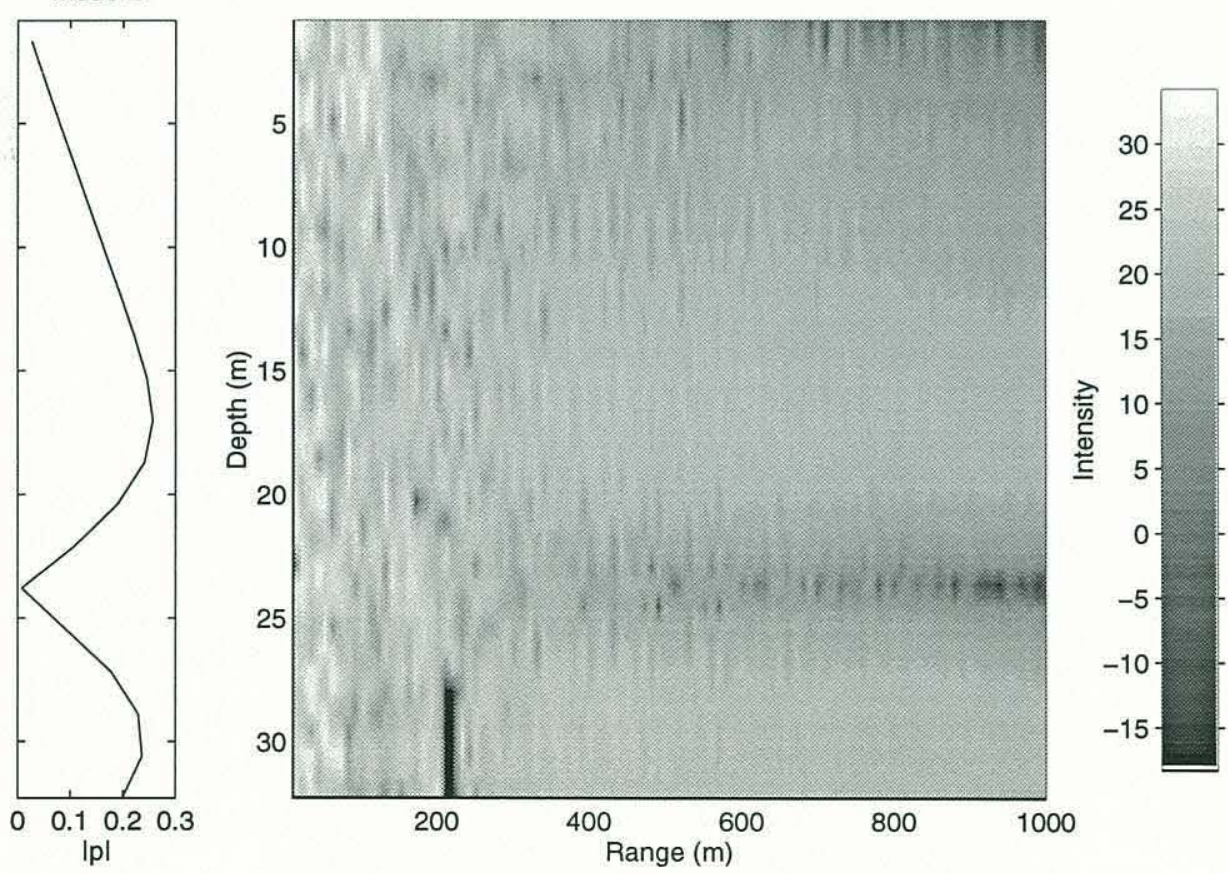

Figure 3-16: Pressure Field Excited by Feedback Controller in Rock Outcropping Environment 
contains several undesired modes when it enters the observation volume. The feedback control algorithm successfully compensates for the coupling introduced by the sloping bottom to excite the desired pressure field at the start of the observation volume.

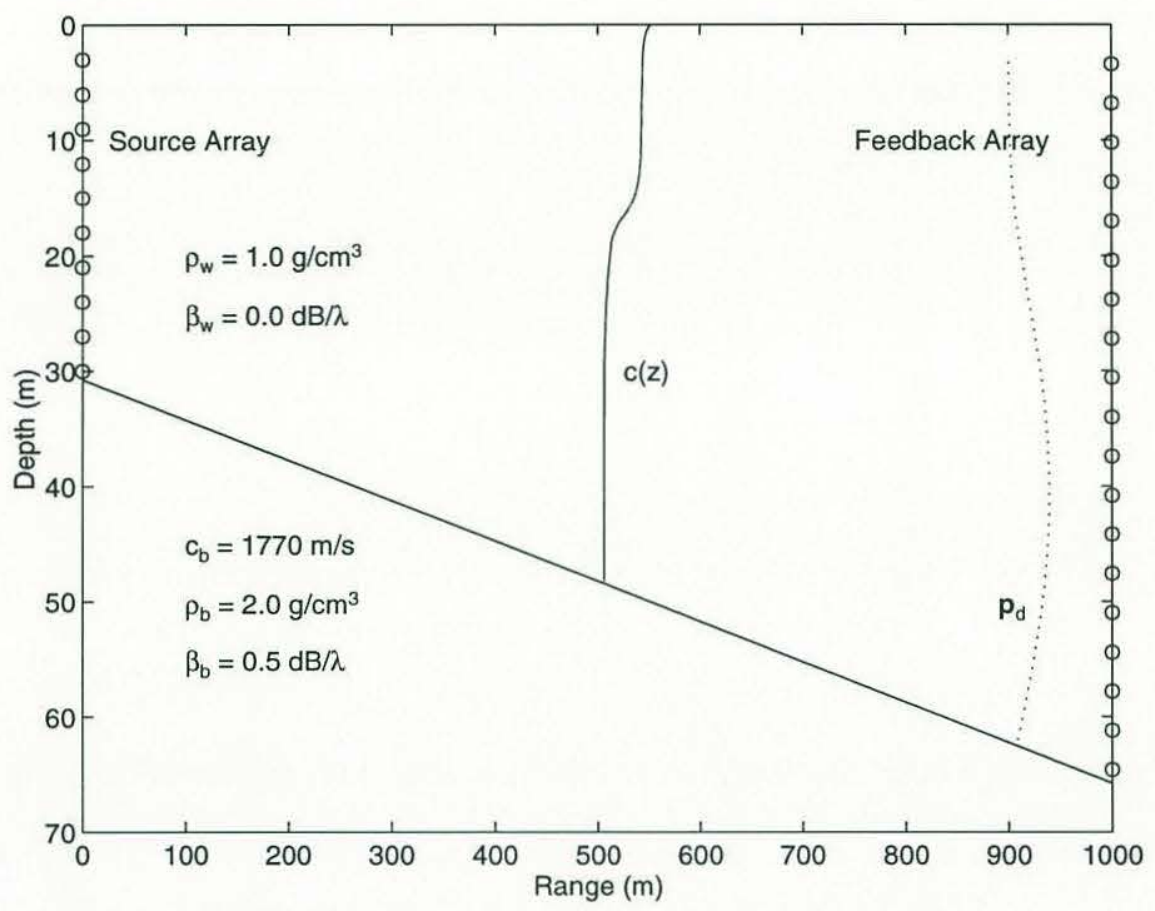

Figure 3-17: Downsloping Wedge Environment.

Figure 3-17 shows the environment used for this simulation. The ten element source array is located at $r=0$, and the nineteen hydrophone feedback array is at $r=1000 \mathrm{~m}$. The depths at the source and feedback array locations are $30.75 \mathrm{~m}$ and $65.75 \mathrm{~m}$, respectively. For the transmission frequency of $400 \mathrm{~Hz}$, the channel supports eight modes at the source array and nineteen modes at the feedback array. The depth at the source array is only slightly shallower than the cutoff depth for mode nine. Mode nine is initially an evanescent mode, but as the channel becomes deeper, it becomes trapped before the energy propagating at that wavenumber has been significantly attenuated. This phenomenon, known as mode capture, has been observed both in laboratory experiments [31] and numerical simulations, [60]. The 
simulations in this section verify that the replica matrix model for propagation used by the feedback control algorithm incorporates continuum effects in propagation sufficiently to model mode capture in downslope propagation. The sound speed profile and other environmental parameters are the same as those used in Section 3.2.

One hundred trials of the feedback control algorithm were run using both the Kalman filter and the CNLLS estimators. The Kalman filter estimator used $\mathbf{P}_{\mathbf{f}}=$ $10^{-6} \mathbf{I}$ and $\alpha=0.99$ and the CNLLS used a forgetting factor giving an effective

window length of 100 iterations. Each trial was initialized with a random $\hat{\mathbf{Q}}[0]$ and was allowed to run for 250 iterations attempting to excite mode one. The observation noise was spatially white and $20 \mathrm{~dB}$ below the energy of the desired signal, $\mathbf{p}_{d}$. Figure 3-17 shows the desired pressure field shape as a dotted line near the feedback array. Figure 3-18 shows the mean SER for these trials, as well as the performance of the open loop algorithm. The open loop algorithm chooses the source array weights to excite mode 1 assuming the mode would propagate adiabatically downslope from the source array. The figure shows that both the Kalman filter and CNLLS estimators work well, but the error in the open loop controller exceeds the energy of the desired signal. Figure 3-19 plots the vertical profile of pressure magnitude for typical trials using each estimator and the open loop algorithm. Regardless of which channel estimator is used, the pressure field generated by the feedback controller is a closer approximation to the desired pressure profile than the field generated by the open loop controller.

\subsection{Solitary Internal Wave}

This section examines the performance of the feedback control algorithm in a simulated environment with a propagating solitary internal wave. The previous simulations in this chapter studied environments where time-invariant mode coupling was induced by bathymetric features such as a sloping bottom or rock outcropping. One 


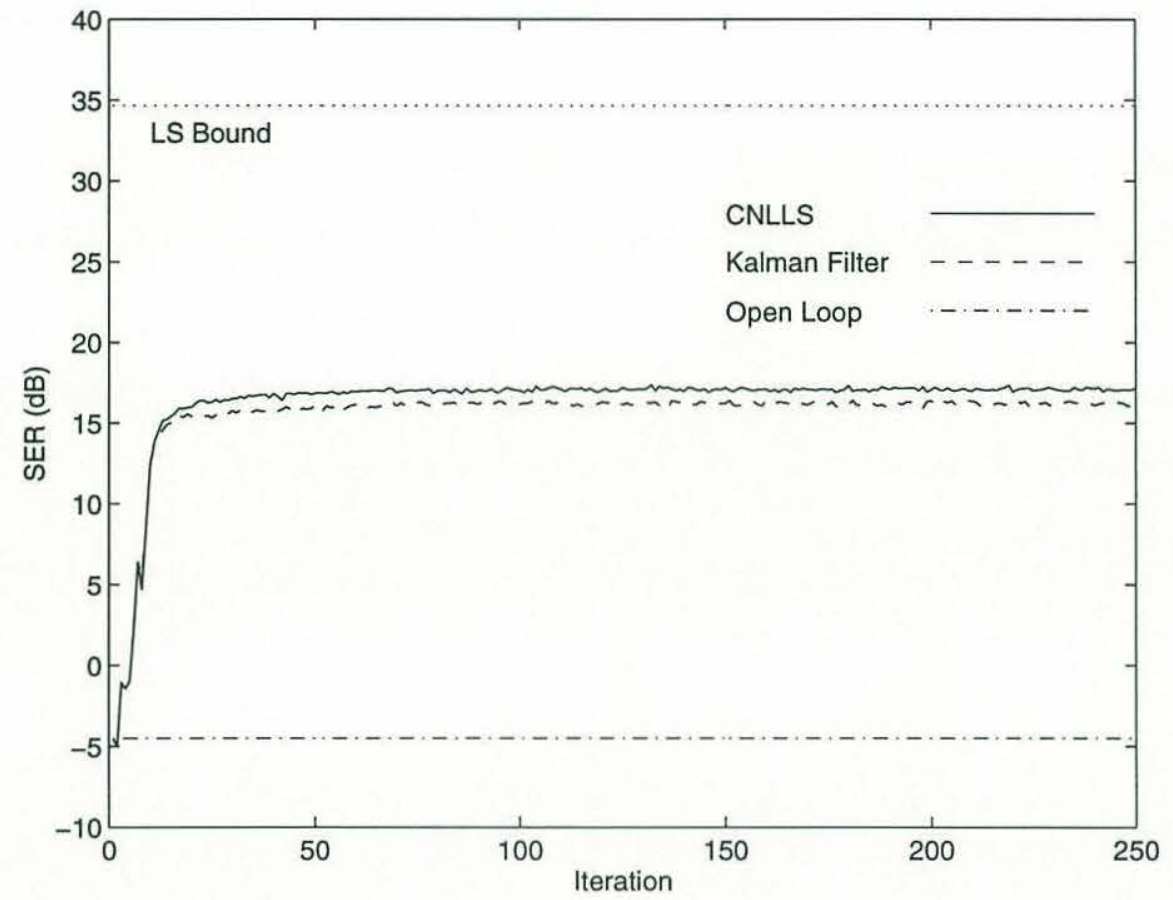

Figure 3-18: Mean SER Convergence for Downsloping Wedge Environment

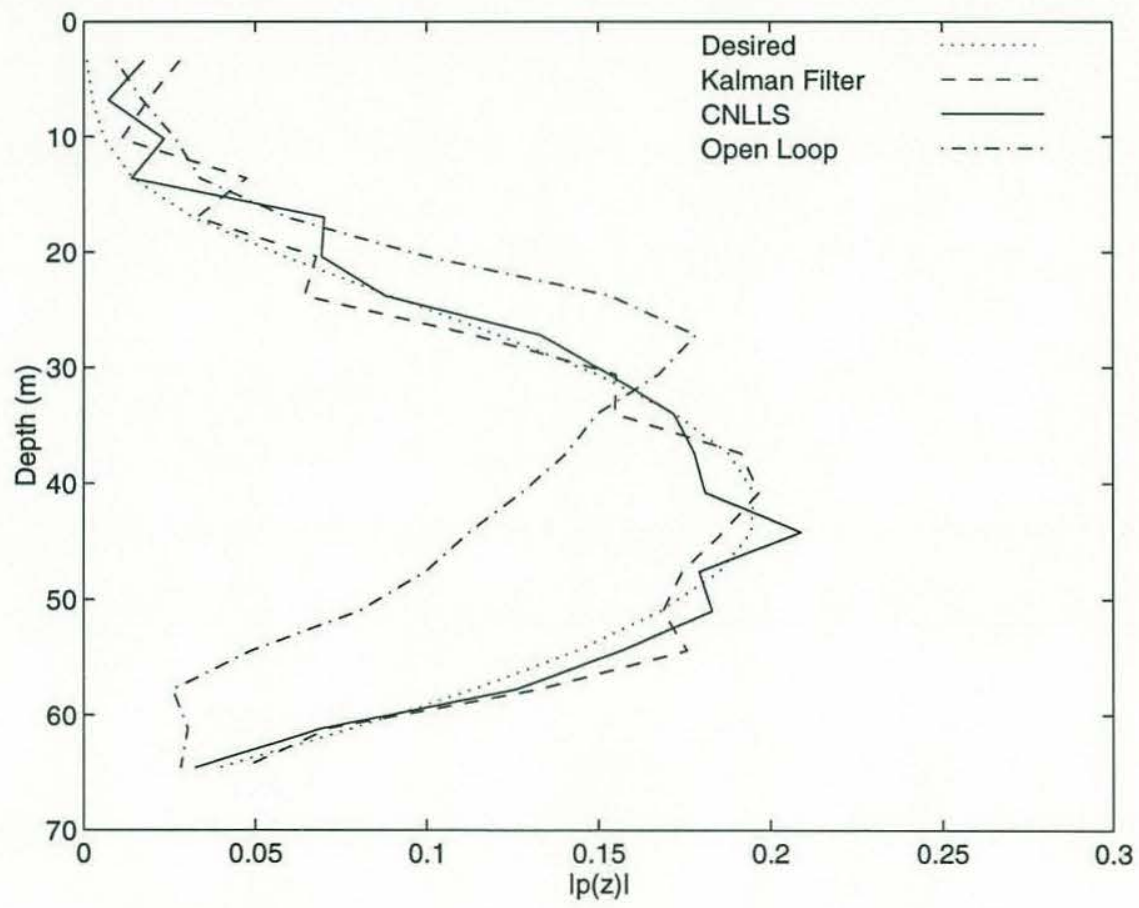

Figure 3-19: Typical Vertical Pressure Profiles for Downsloping Wedge Environment 
of the primary motivations for developing a single mode source such as the feedback control algorithm is to study the acoustic coupling introduced by internal waves in the observation volume. If the feedback control algorithm is to function successfully as a single mode source in such an environment, it must be capable of exciting a single mode even when solitary waves are propagating through the feedback volume. This simulation models the time-varying acoustic mode coupling between the source and feedback arrays introduced by a solitary internal wave propagating through the feedback volume. Based on studies of internal waves supported on density gradients in coastal regions [61] [62] [63], the thermocline at $z=10 \mathrm{~m}$ of the simulated environment is displaced by $10 \operatorname{sech}^{2}\left((r-1300+t) k_{\text {int }}\right) \mathrm{m}$ for $1000 \leq r \leq 1600$, where the horizontal wavenumber of the internal wave is $k_{i n t}=(2 \pi / 300) \mathrm{m}^{-1}$. Starting with a typical sound speed profile observed in [58], this displacement profile gives the sound speed profile shown in Fig. 3-20. In this figure, lighter regions indicate water with a higher sound speed, while darker regions indicate colder water with a slower sound speed.

The source array is located at $r=0 \mathrm{~m}$, and the feedback array at $r=1000 \mathrm{~m}$. The solitary wave propagates towards $r=0 \mathrm{~m}$ with a velocity of $1 \mathrm{~m} / \mathrm{s}$, consistent with the velocities observed for these waves in [61]. The replica matrices were computed every $10 \mathrm{~m}$ (or equivalently every 10 seconds) using FEPE. The frequency is $400 \mathrm{~Hz}$, which gives nine trapped modes for the water depth of $34 \mathrm{~m}$. The simulation used the same bottom parameters and source and feedback arrays as Section 3.1. The feedback control algorithm attempts to excite mode two starting from a randomly chosen $\hat{\mathbf{Q}}[0]$ and using an iteration time of $1 \mathrm{sec}$. The slowest mode in this environment propagates from the source array to the feedback array in roughly $0.8 \mathrm{sec}$, so this choice of iteration time insures the field observed at the feedback array reflects the most recent source array weights. The observation noise is again spatially white and $20 \mathrm{~dB}$ below the pressure profile for mode two.

The simulation was run with both the CNLLS and Kalman Filter channel estima- 

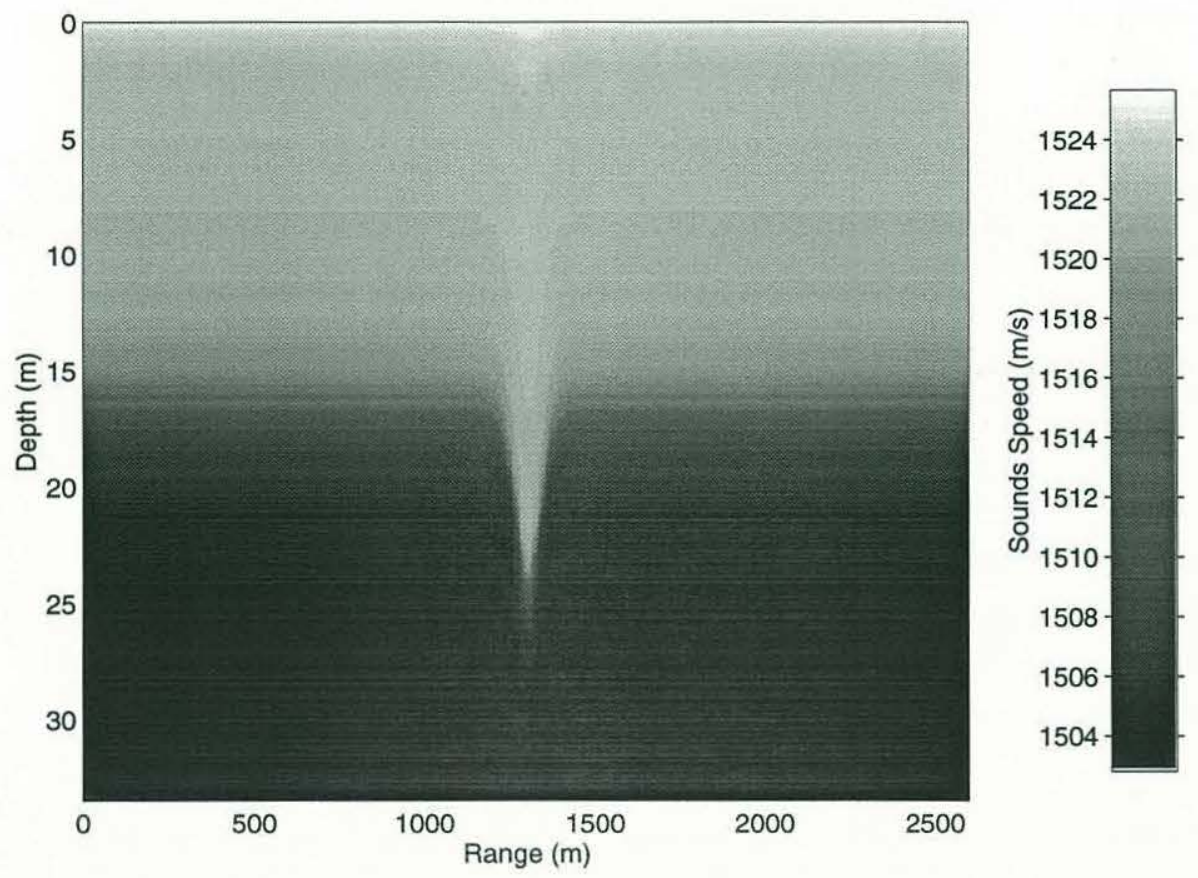

Figure 3-20: Sound speed profile $c(r, z)$ for solitary internal wave with $\operatorname{sech}^{2}$ profile

tors. The former used a forgetting factor of $\gamma=0.9772$, giving an effective window length of 100 iterations. The Kalman filter estimator set $\alpha=0.99$ and $\mathbf{P}_{\mathbf{f}}=10^{-6} \mathbf{I}$. Figures 3-21 and 3-22 show the performance of the feedback control algorithm using the CNLLS and Kalman filter estimators, respectively. Because the internal wave is propagating with velocity $1 \mathrm{~m} / \mathrm{s}$, the time axes of Figs. 3-21 and 3-22 can also be interpreted as the initial range location in Fig. 3-20 of the sound speed profile currently located at the source array. Each figure also shows the performance of the open loop algorithm in the same environment.

Figures 3-21 and 3-22 clearly demonstrate the feedback control algorithm using either estimator performs better than the open loop algorithm in exciting the desired mode. The open loop control algorithm observes the initial sound speed profile at the source array, and assumes the feedback volume is range-invariant with this profile throughout the experiment. Initially, this assumption is valid and attains the limit on SER imposed by the observation noise. Once the solitary wave starts entering 


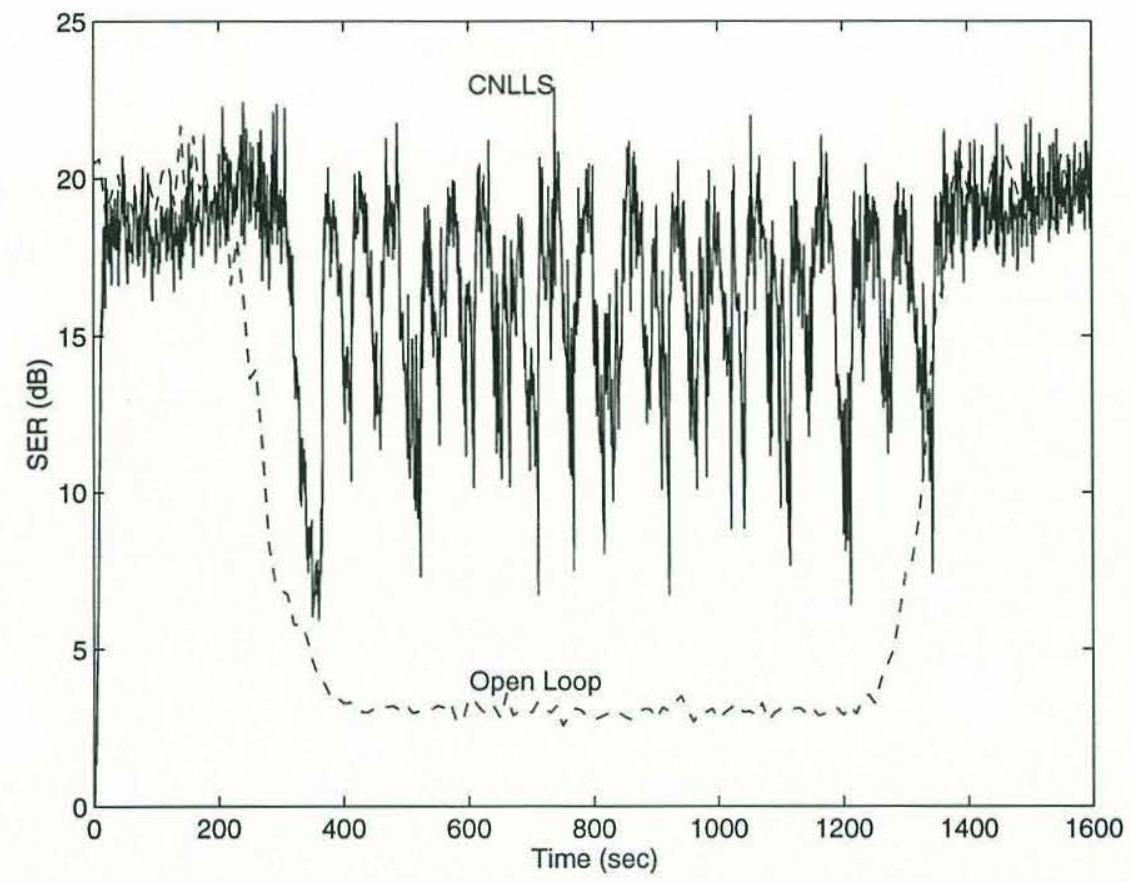

Figure 3-21: Performance of feedback control algorithm using CNLLS estimator compared with open loop controller for solitary wave propagating through feedback volume.

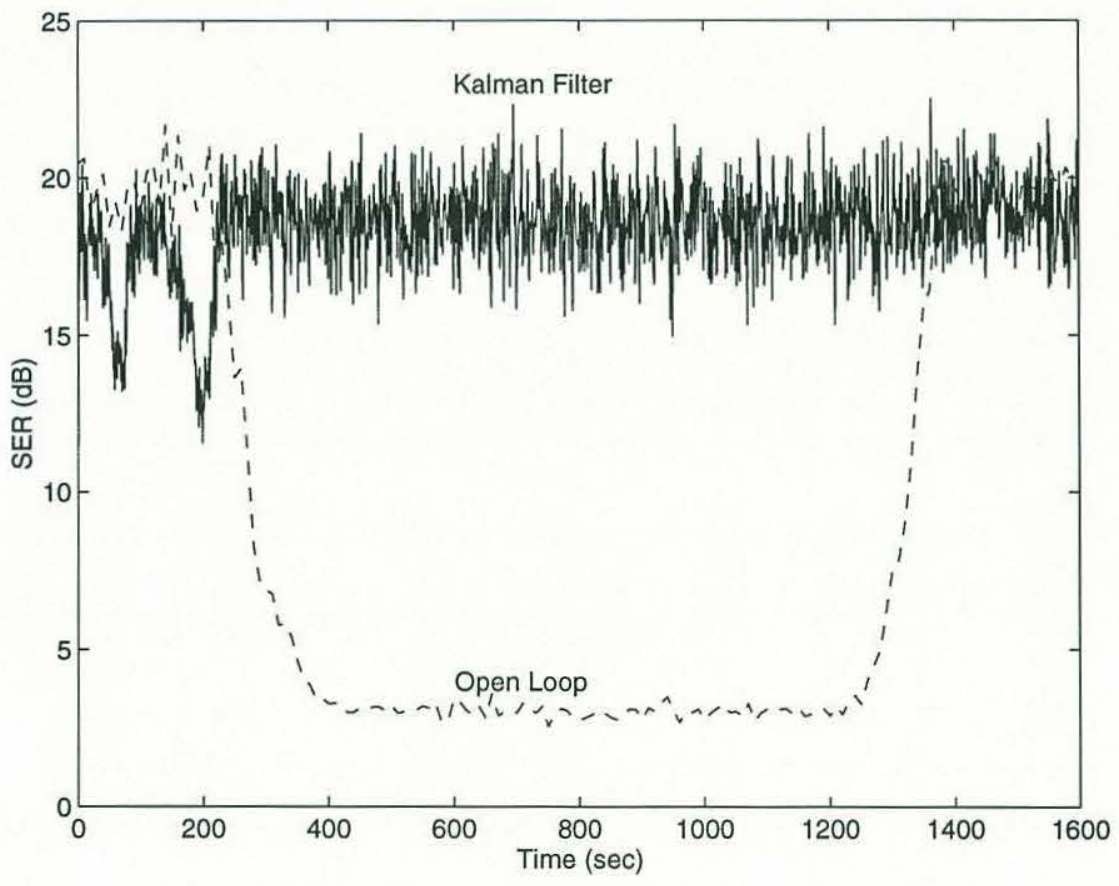

Figure 3-22: Performance of feedback control using Kalman filter estimator compared with open loop controller for solitary wave propagating through the feedback volume. 
the feedback volume the open loop algorithm's performance deteriorates severely and does not recover until the wave has largely propagated past the source array. The Kalman filter, in particular, tracks the time-varying coupling introduced by the solitary wave to maintain a consistently high fidelity mode after an initial transient. The pressure at the feedback array for the CNLLS has significant oscillations, making its pressure field a less desirable source for downrange observers than the field generated by the Kalman filter. The CNLLS algorithm assumes the dynamics of the rows of the replica matrix are stationary over the length of the effective window introduced by the forgetting factor. The deterministic propagation of the solitary wave through the feedback volume violates this assumption. Simulations run with time-invariant environments chosen from the replica matrices representing the solitary wave inside the feedback volume show the CNLLS estimator converges under these conditions. This also suggests the oscillations are caused by the nonstationary environment. The Kalman filter does not assume the replica matrix is stationary, and is able to excite a high fidelity single mode while a strong solitary wave propagates through the feedback volume inducing time-varying coupling.

\subsection{Summary}

This chapter has demonstrated that the feedback control algorithm is a viable method for exciting a single mode in several simulated coastal environments ranging from a simple adiabatic range- and time-invariant channel to a complicated rangeand time-varying propagation environment generated by a propagating solitary internal wave. These encouraging results suggest that the next logical step is to evaluate the performance of the feedback control algorithm in a scale model experiment. Such an experiment will introduce the limitations of real-time hardware and the vagaries of physical acoustic propagation, testing the robustness of the algorithm. The next chapter describes a set of experiments which study the feedback control algorithm 
under these conditions. 


\section{Chapter 4}

\section{Laboratory Waveguide Experiments}

This chapter describes a series of experiments using the feedback control algorithm to control the pressure field in a laboratory waveguide. These experiments investigated the performance of the feedback control algorithm implemented on real-time hardware and with actual rather than simulated acoustic propagation. Section 4.1 describes the laboratory waveguide used for these experiments as well as the transducers and signal processing hardware. The acoustic characteristics of the waveguide are discussed in Section 4.2. The first set of experiments confirmed that the feedback control algorithm could successfully control the pressure field in the waveguide without a priori knowledge of the propagation characteristics. Section 4.3 describes these experiments as well as a second series of experiments that explored which parameters of the channel estimators gave the best performance in the waveguide. Section 4.4 presents the results of a series of experiments investigating the transient response of the feedback algorithm when an acoustic scatterer was introduced into the waveguide after the algorithm had been allowed to converge. 


\subsection{Waveguide and Equipment Description}

The laboratory waveguide, or flume, used for these experiments was originally designed for fluid dynamics rather than acoustics experiments. Consequently, the flume is not an ideal acoustic waveguide, and little information is available about the aspects of construction germane to its acoustic properties. Much of what follows about the materials or structure is conjecture based on inspection of the flume, since no records or plans exist from the waveguide's construction. The tank is roughly $20 \mathrm{~m}$ long, $1 \mathrm{~m}$ deep and $1.2 \mathrm{~m}$ wide from front to back. The front face is constructed of plates of 3/8-inch thick plexiglass, joined by aluminum brackets to each other and $10 \mathrm{~cm}$ thick concrete pilings roughly every $2 \mathrm{~m}$. Fortunately, the distance the pilings project into the flume is insignificant compared to wavelength of these experiments. The back wall appears to be constructed of concrete roughly $10 \mathrm{~cm}$ thick. The back wall and bottom of the flume were thickly painted or coated with a latex-type material in a manner that left large $(20-30 \mathrm{~cm})$ bubbles irregularly spaced in several locations on the bottom. The bottom appeared to be largely solid concrete but a strip of significant width $(30-40 \mathrm{~cm})$ sounded hollow, perhaps fiberglass over a channel containing plumbing for the pumps used to generate hydrodynamic flows in the tank.

Two baffling plates were installed at either end of the tank for these experiments to reduce the amount of energy reflected, making it a closer approximation to an infinitely long waveguide. Each baffler was a steel plate covered with a layer of VRC PB 1-94 anechoic tiles manufactured by Vector Research Corporation under contract from Seaward International and generously loaned to us for these experiments. This material consists of polyurethane encapsulated gaseous microspheres, and was molded into pyramids roughly $2 \mathrm{~cm}$ square at the base and extending $6 \mathrm{~cm}$ out from the plate. The material was designed for $20 \mathrm{kHz}$, rather than the $8 \mathrm{kHz}$ operating frequency used in these experiments. Preliminary calibration data from Vector Research indicates even at these lower frequencies the panels should still provide 
roughly $10 \mathrm{~dB}$ of echo reduction at this lower frequency. The flume was filled with fresh water to a depth of $66 \mathrm{~cm}$ at the source array, which gave a depth of $69 \mathrm{~cm}$ at the feedback array due to a slight slope in the bottom of the flume. Assuming a frequency of $400 \mathrm{~Hz}$ in the ocean experiment, scaling these depths by the same factor of twenty as the frequency indicates this would be equivalent to a 13-14 m water depth for an ocean experiment.

The source array consisted of six Channel Industries 3013 ceramic cylinder 8$15 \mathrm{kHz}$ acoustic sources, spaced $10 \mathrm{~cm}$ apart and offset $5 \mathrm{~cm}$ from the surface of the water. The feedback array consisted of seven Benthos AQ2TS hydrophones spaced every $8 \mathrm{~cm}$. The hydrophones, along with their pre-amplifiers, were sealed in a polyurethane tube filled with mineral oil to minimize the acoustic impedance mismatch with the surrounding water. A tapered lead weight ballasted the base of the feedback array, allowing it to be tensioned to remain vertical. The pre-amplifiers were calibrated before insertion into the array so that all the pre-amplifiers had gains of $36.5 \pm 0.1 \mathrm{~dB}$ per WHOI standard [64]. Figure 4-1 provides a schematic of the physical layout of the waveguide for the experiments. The arrays were centered width-wise in the waveguide, roughly $60 \mathrm{~cm}$ from the front and back walls. If the source array is defined as the origin in range, with range increasing towards the feedback array, the baffling plates were located at $r=-1.6 \mathrm{~m}$ and $r=12.95 \mathrm{~m}$. The feedback array was located at $r=10.9 \mathrm{~m}$.

The feedback control algorithm was implemented in the Acoustic Modem System (AMS-2.0), a MATLAB-like real-time signal processing environment for the Texas Instrument TMS320C40 DSP chip. AMS was developed by Mark Johnson and Matt Grund of the Acoustic Telemetry Group at the Woods Hole Oceanographic Institution to assist rapid development of real-time signal processing applications in underwater acoustics [65]. The A/D and D/A conversion required for measuring the pressure at the feedback array and transmitting the source array weights were handled by two of the modems also developed by the Acoustic Telemetry Group. 


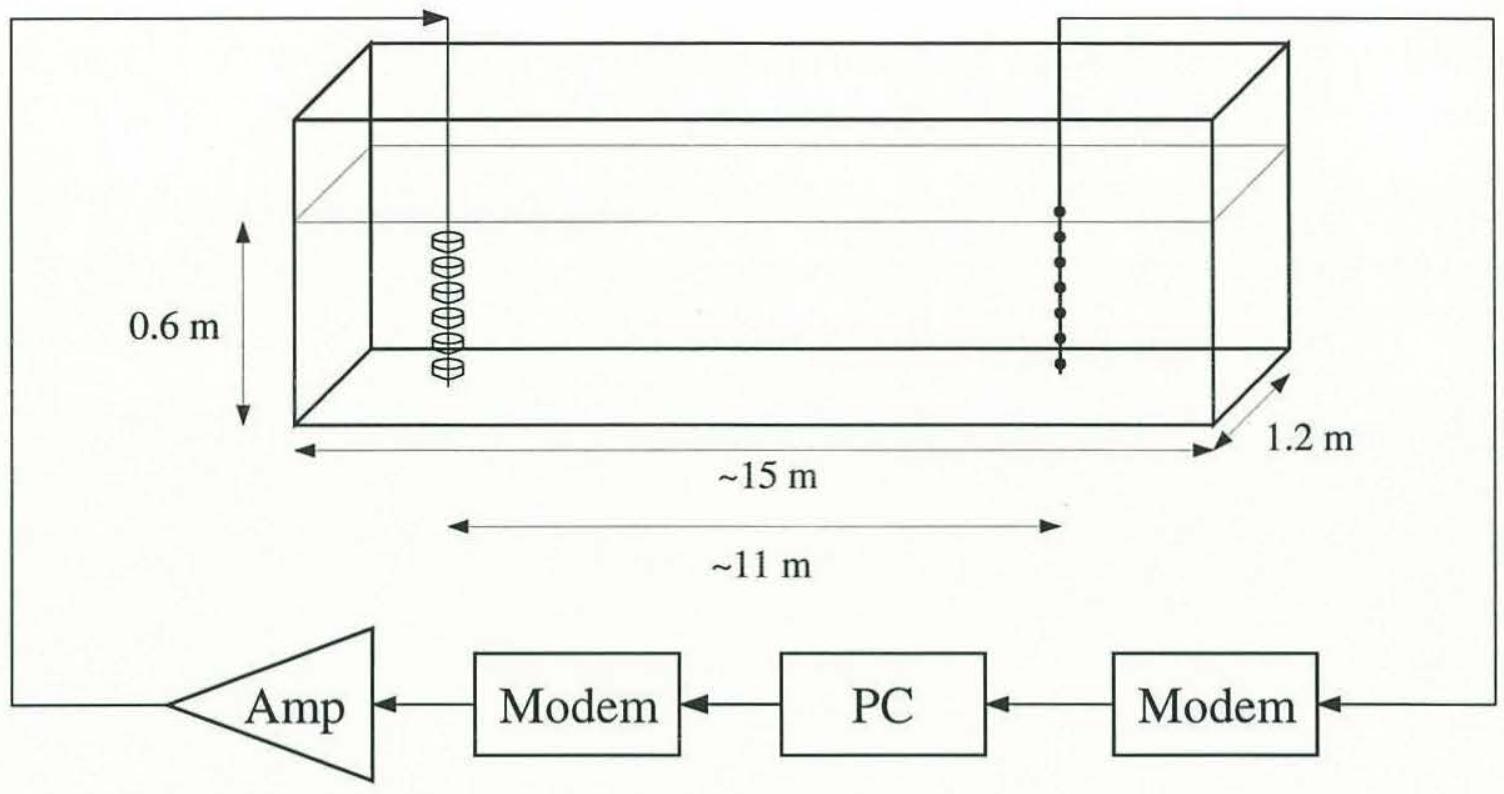

Figure 4-1: Configuration of Laboratory Waveguide Experiments

One modem sampled the pressures observed at the hydrophone array, while the second modulated the complex source array weights onto narrowband sinusoids. These sinusoids were fed to six independent power amplifiers, which drove the source array.

\subsection{Acoustic Properties of the Laboratory Waveg- uide}

This section describes the acoustic properties of the laboratory waveguide for the experimental configuration outlined in Section 4.1. The water depth in the waveguide was $66 \mathrm{~cm}$ at the source array and the frequency was $8 \mathrm{kHz}$. The temperature of the fresh water used to fill the waveguide was consistently $25^{\circ} \mathrm{C}$, which gives a sound speed of $1490 \mathrm{~m} / \mathrm{s}$. The ideal isovelocity constant-depth waveguide with a pressure-release surface and rigid bottom [22] supports six trapped modes for this water depth and frequency. The bottom of the flume is not a homogeneous perfect acoustic reflector, making the flume unlikely to be a good approximation to this 
ideal waveguide. In addition, the boundary conditions imposed across the width of the flume by the plexiglass front wall and back wall of unknown composition further complicate the acoustic propagation. Since the flume has a rectilinear geometry, the width and depth eigenfunctions of the waveguide should be separable. The width eigenfunctions should superimpose at the location of the vertical feedback array to form modes with the vertical profiles of the depth eigenfunctions.

Preliminary experiments verified that the acoustic propagation between the source array and feedback array was well modeled by an LTI system. Sequential excitation and scaling of source array elements confirmed the channel satisfied linearity. Long term experiments using the same set of source array weights indicated the channel was effectively time-invariant, with a correlation time on the order of hours when the water in the waveguide was calm. These experiments also indicated that the observation noise present in the system was more than $40 \mathrm{~dB}$ below the desired signal levels.

The sequential excitation experiments also provided an estimate of the replica matrix for the waveguide. Computing the SVD of this matrix revealed that only three modes of the system accounted for over $99 \%$ of the energy received at the feedback array. Figures 4-2 and 4-3 plot the singular values $\left(\sigma_{\hat{\mathbf{Q}}}\right)$ and pressure modes $\left(\mathbf{U}_{\hat{\mathbf{Q}}}\right)$ for the first four modes of the estimated replica matrix $\hat{\mathbf{Q}}$. The very high SNR in the waveguide means this should be an accurate estimate of the true replica matrix. For the remainder of the discussion in this chapter, the replica matrix estimated by sequentially exciting the sources will be considered to be the true $\mathbf{Q}$, and the caret $(\hat{*})$ will be reserved for replica matrices estimated by the feedback control algorithm. In general, the columns of $\mathbf{U}_{\mathbf{Q}}$ will not equal the sampled mode shapes, but they should span the same space as the acoustic modes. Projecting the theoretical modes shapes for an isovelocity waveguide with a rigid bottom onto the observed $\mathbf{U}_{\mathbf{Q}}$ will indicate how well these theoretical modes fall in the observed span of the system. Performing this projection reveals a significant portion of the energy in the first three theoretical 
modes falls outside the span of the first three columns of $\mathbf{U}_{\mathbf{Q}}$. This indicates the laboratory waveguide is not a good approximation to an isovelocity waveguide with a rigid bottom. This is not surprising given the unknown composition and irregular construction of the bottom of the flume.

Repeated estimations of $\mathbf{Q}$ indicate the tank is well-modeled as LTI over the time-scales considerably longer than the propagation time between the source and feedback arrays. Although the singular values do vary slowly over time, they remain roughly the same size as those shown in Fig. 4-2. Based on the values plotted in Fig. 4-2, three, or at most four, modes propagate in the laboratory waveguide.

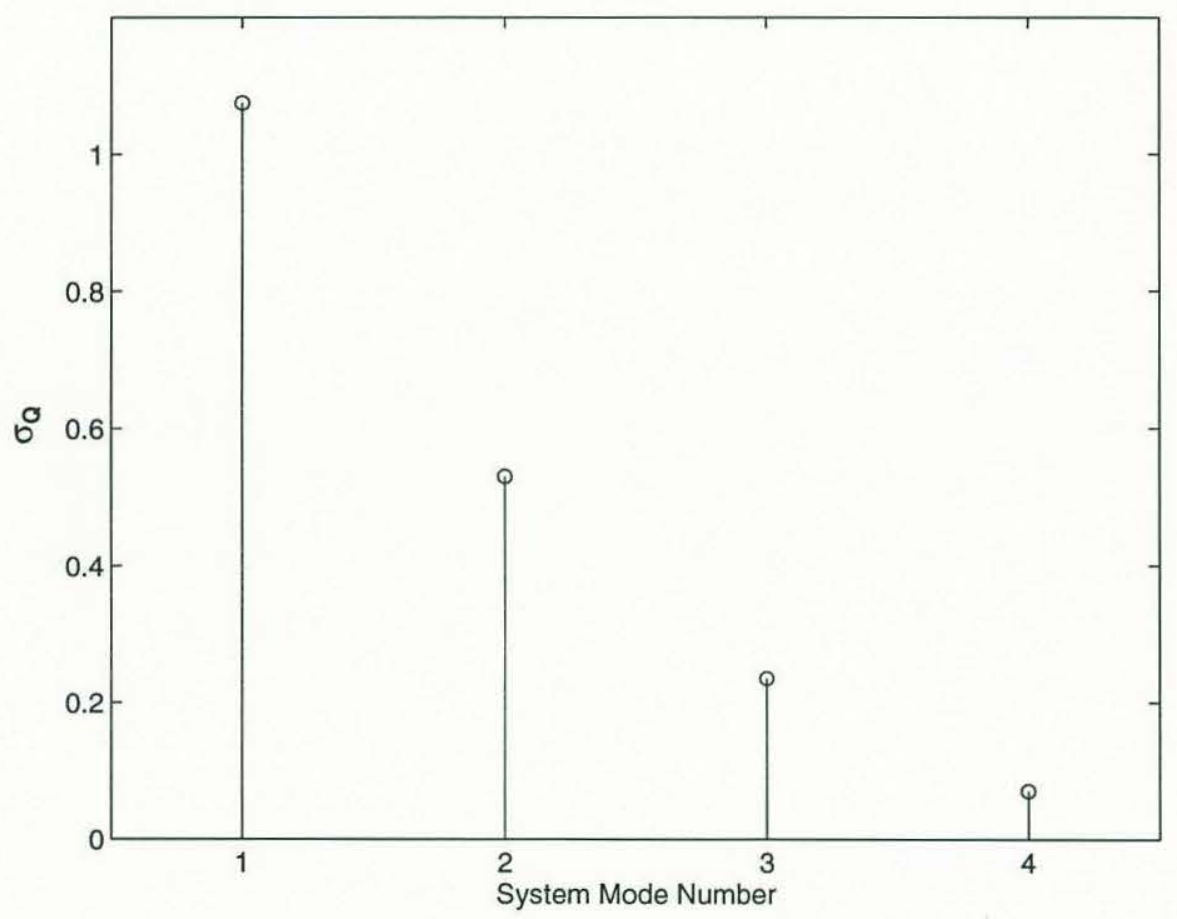

Figure 4-2: Singular Values of Replica Matrix for Laboratory Waveguide

The remainder of the experiments described in this chapter use the columns of $\mathbf{U}_{\mathbf{Q}}$, or system modes, for the desired pressure profile. In a well-designed ocean experiment, the acoustic modes fall within the span of the columns of $\mathbf{U}_{\mathbf{Q}}$, allowing the feedback control algorithm to excite the desired mode. Demonstrating the algorithm is capable of obtaining pressure profiles in the numerical span of $\mathbf{Q}$ in the 


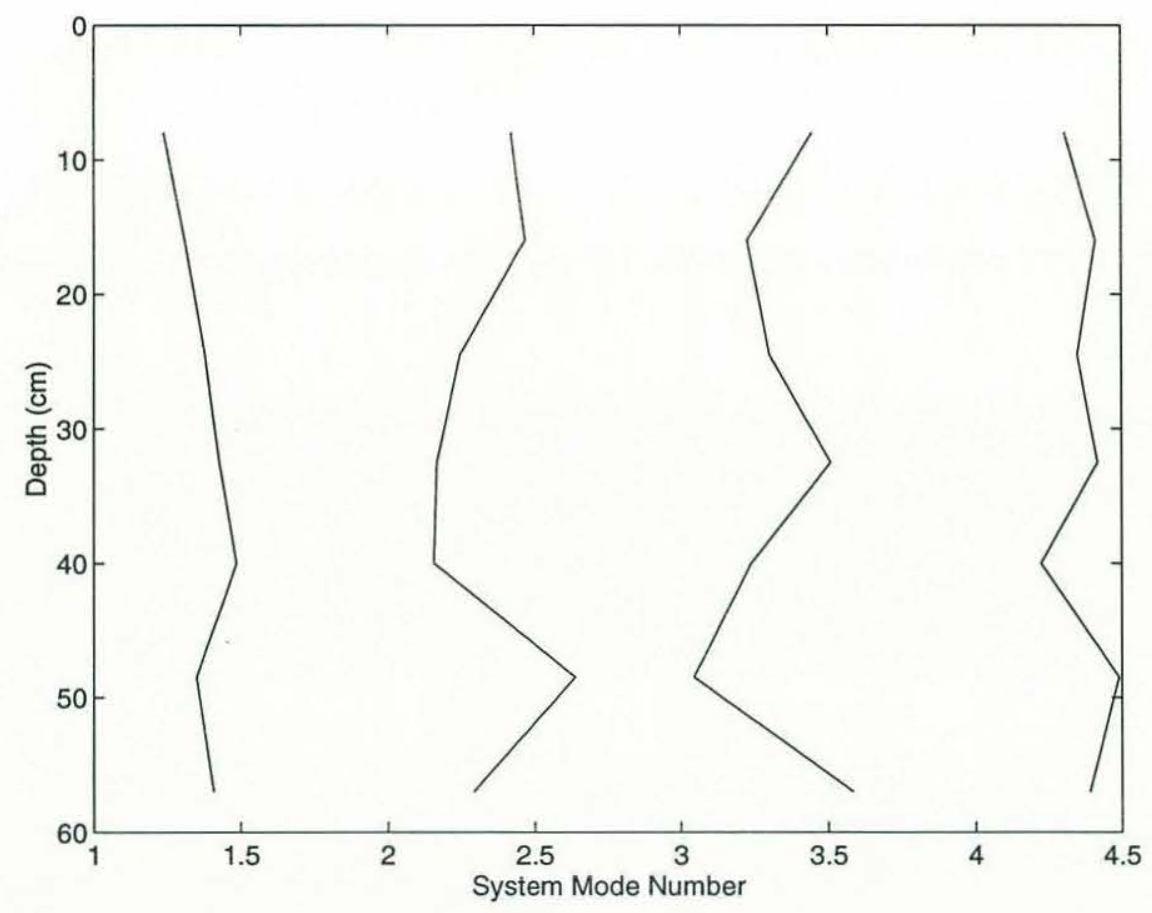

Figure 4-3: Pressure Magnitude for System Modes of Laboratory Waveguide

flume should give us confidence that the algorithm will be successful in exciting the desired acoustic mode in a well-designed ocean experiment.

\subsection{Time-Invariant Waveguide}

The experiments in this section demonstrate the feedback control algorithm can successfully excite the desired pressure profile in a time-invariant laboratory waveguide. The initial $\mathbf{Q}[0]$ is the true $\mathbf{Q}$ with noise added $20 \mathrm{~dB}$ below the true values. Starting with this initial $\hat{Q}[0]$, the feedback control algorithm was allowed to run for 100 iterations with a 0.9 second iteration time. The desired pressure profiles were the system modes, i.e., the columns of $U_{\mathbf{Q}}$. The experiments were run on a different day with a slightly different $\mathbf{Q}$ than the replica matrix measured in the last section. As a result, the desired pressure profiles and relative energies were similar but not identical to those shown in Figs. 4-3 and 4-2. The gains of the desired pressure profiles were 
scaled to prevent saturation of either the source array D/A or the feedback array A/D. Both the Kalman filter and CNLLS estimators were used in these experiments. The Kalman filter used $\alpha=1.0$ and $\mathbf{P}_{\mathbf{f}}=10^{-5} \mathbf{I}$; the CNLLS used a forgetting factor of $\gamma=0.9550$ and limited the condition number of $\Phi^{-1}[n]$ to 200. This limit on the condition number is much more severe than the $10^{6}$ used in the simulations of Chapter 3 due to numerical sensitivities in the eigenvalue library implemented in single precision on the TMS320C40. Experiments described later in this section explore other parameter choices for the channel estimators and confirm that although these may not be the ideal parameter choices for the estimators they are reasonable. Figure 4-4 shows the performance of the feedback control algorithm exciting system mode one. Both estimators give a very high SER: the Kalman filter estimator attains nearly $50 \mathrm{~dB}$ of SER, while the CNLLS estimator levels out around $45 \mathrm{~dB}$ SER. The Kalman filter experiment shows several drop-outs of the signal. These were caused by network delays during the experiment. The transmitter and receiver modems used a semaphore protocol via files on the NFS server to synchronize the sampling of the pressure field at the feedback array. Unfortunately, network delays sometimes disrupted this synchronization and the receiver did not sample the pressure until after the source array stopped transmitting. These glitches are regrettable, but they do allow the Kalman filter estimator to demonstrate its robustness to this sort of error. Other experiments shown later in the chapter indicate the CNLLS and LMS algorithms are also robust to this sort of error. This is encouraging for the ocean experiments because the radio telemetry link shown in Fig. 1-1 between the feedback array and source ship will likely experience similar intermittent disruptions.

Figures 4-5 through 4-7 show the performance of both estimators for system modes two through four. All of these experiments demonstrate that the feedback algorithm is capable of exciting high fidelity modes. The fidelity of the excited pressure profile for mode four is particularly impressive, since the singular value corresponding to this system mode is more than $20 \mathrm{~dB}$ below the singular value 


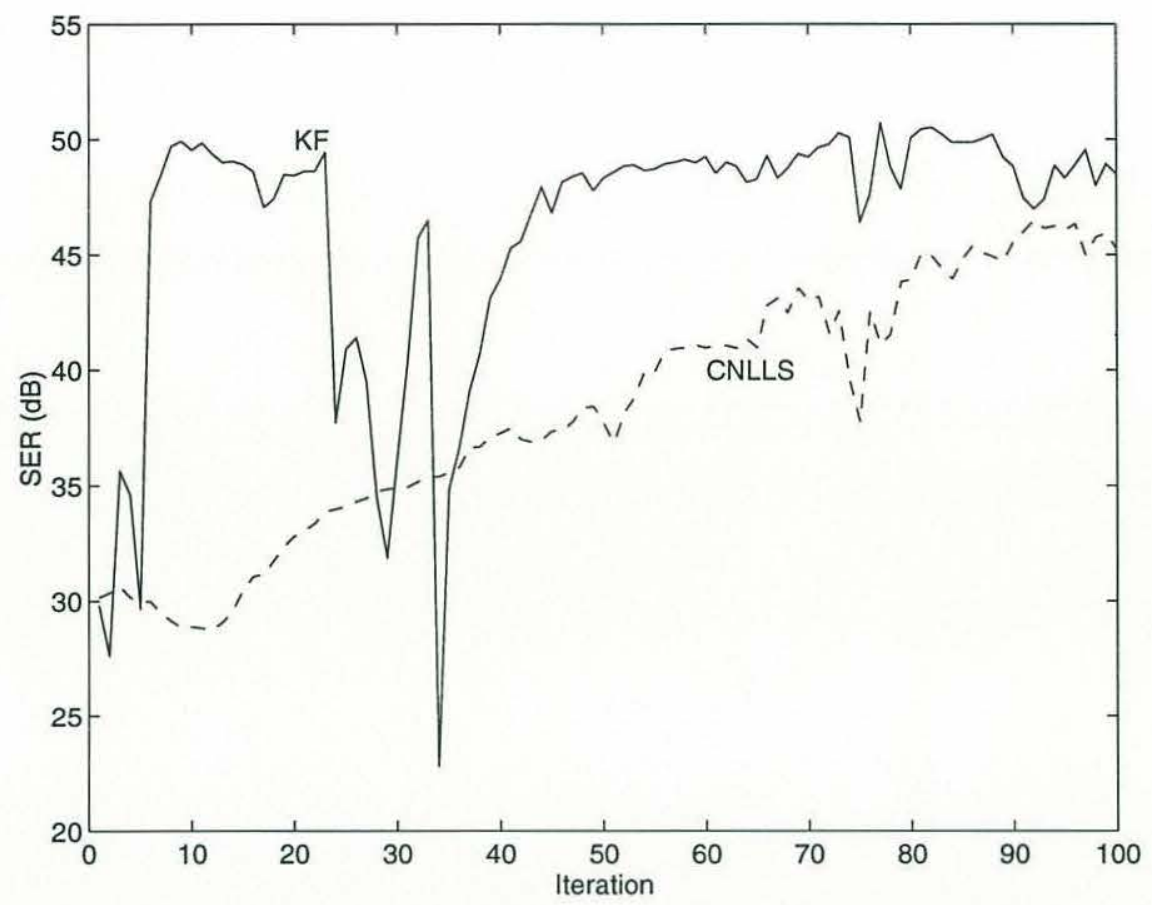

Figure 4-4: SER Performance for System Mode 1 in Time-Invariant Waveguide

for mode one. This can be seen in Fig. 4-2, where the magnitude of the pressure profile of system mode four is far below that of system mode one. The performance for mode four shown in Fig. 4-7 indicates this mode is both numerically reachable and observable for the conditions in the flume. Figure 4-8 shows the performance of the feedback control algorithm when the desired pressure profile was system mode one plus $0.4 j$ times system mode two. The high SER for this experiment confirms the algorithm can excite linear combinations of the system modes as well as the individual modes. If the desired acoustic mode in an ocean experiment is not one of the system modes but falls within the appropriate numerical span of the system modes, the feedback control algorithm should be able to excite the pressure profile of the acoustic mode with high fidelity.

Figures 4-9 through 4-11 show the performance of the channel estimators with different choices of parameters. All the experiments shown in these figures started with no a prior $i$ knowledge of $\mathbf{Q}$, i.e., random $\hat{\mathbf{Q}}[0]$, and attempted to excite system 


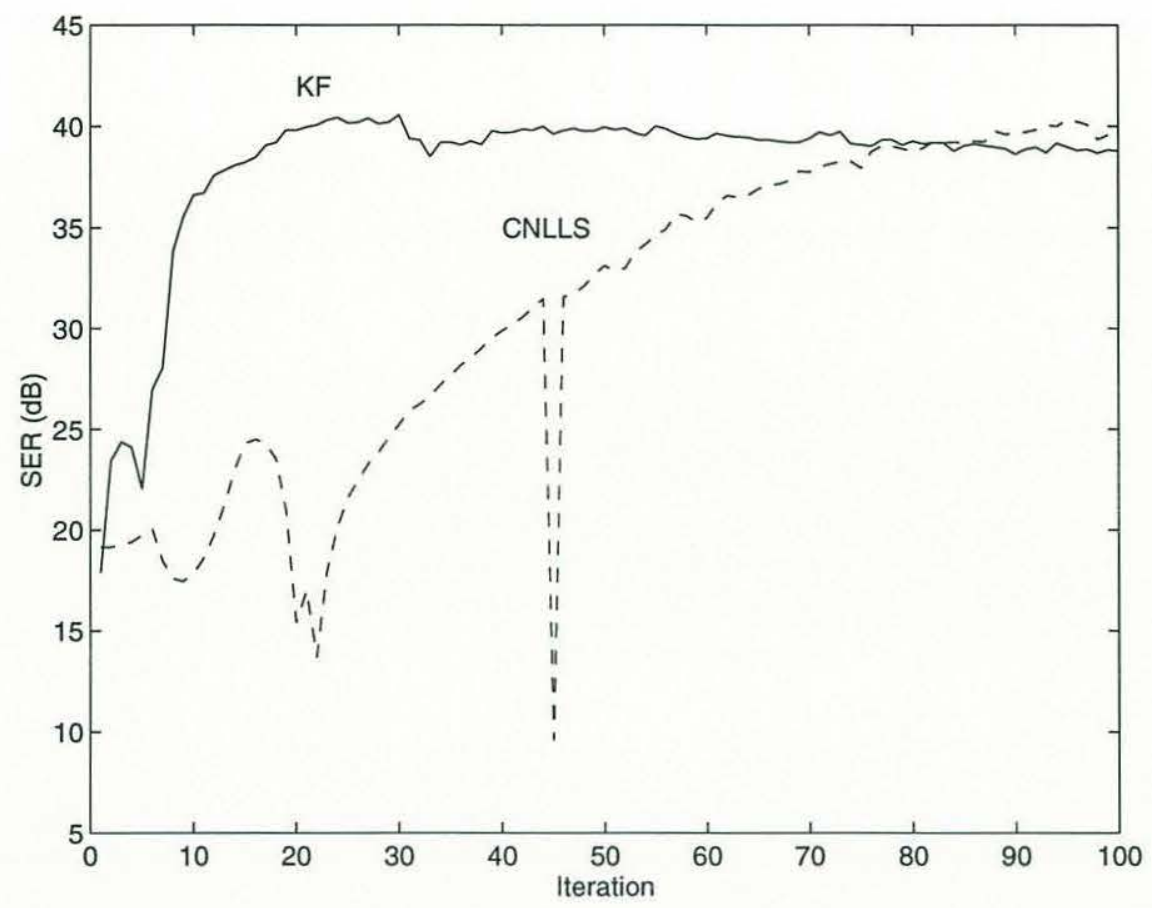

Figure 4-5: SER Performance for System Mode 2 in Time-Invariant Waveguide

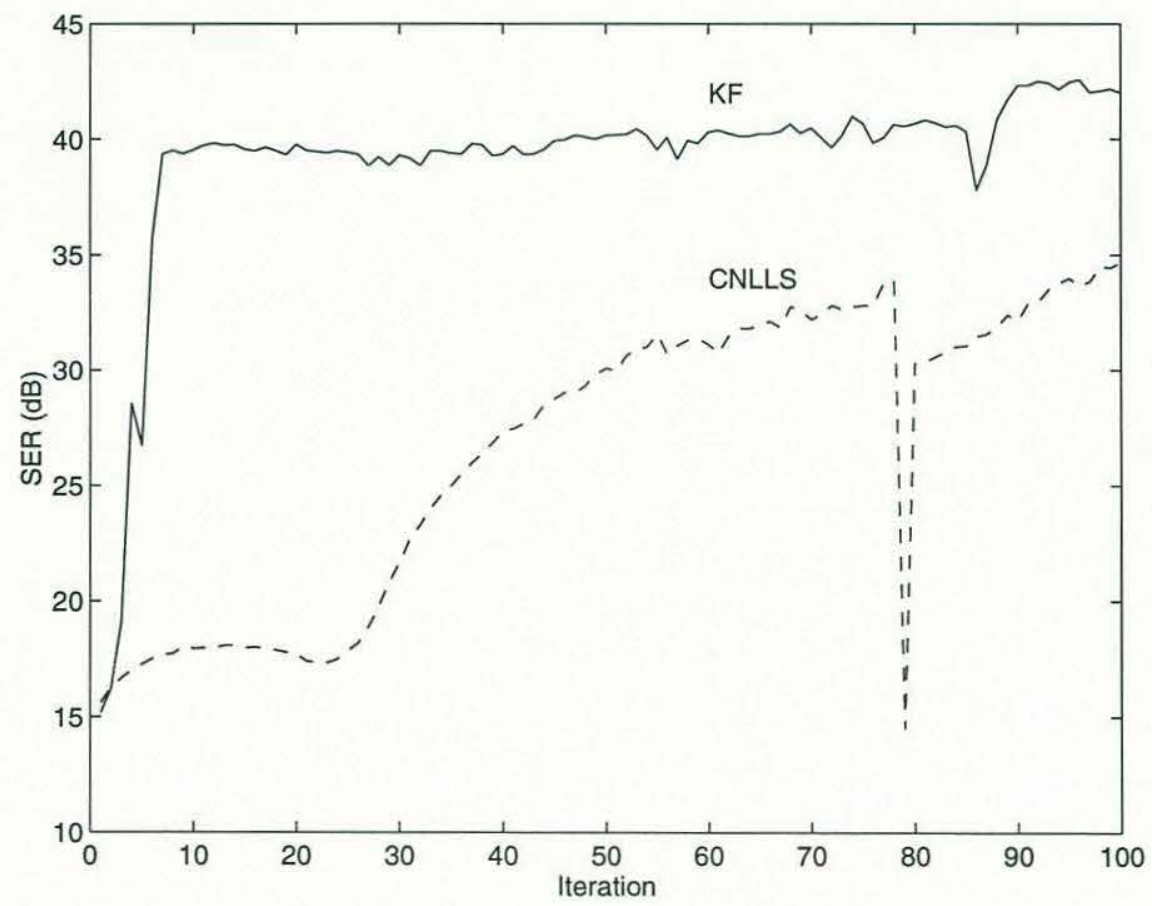

Figure 4-6: SER Performance for System Mode 3 in Time-Invariant Waveguide 


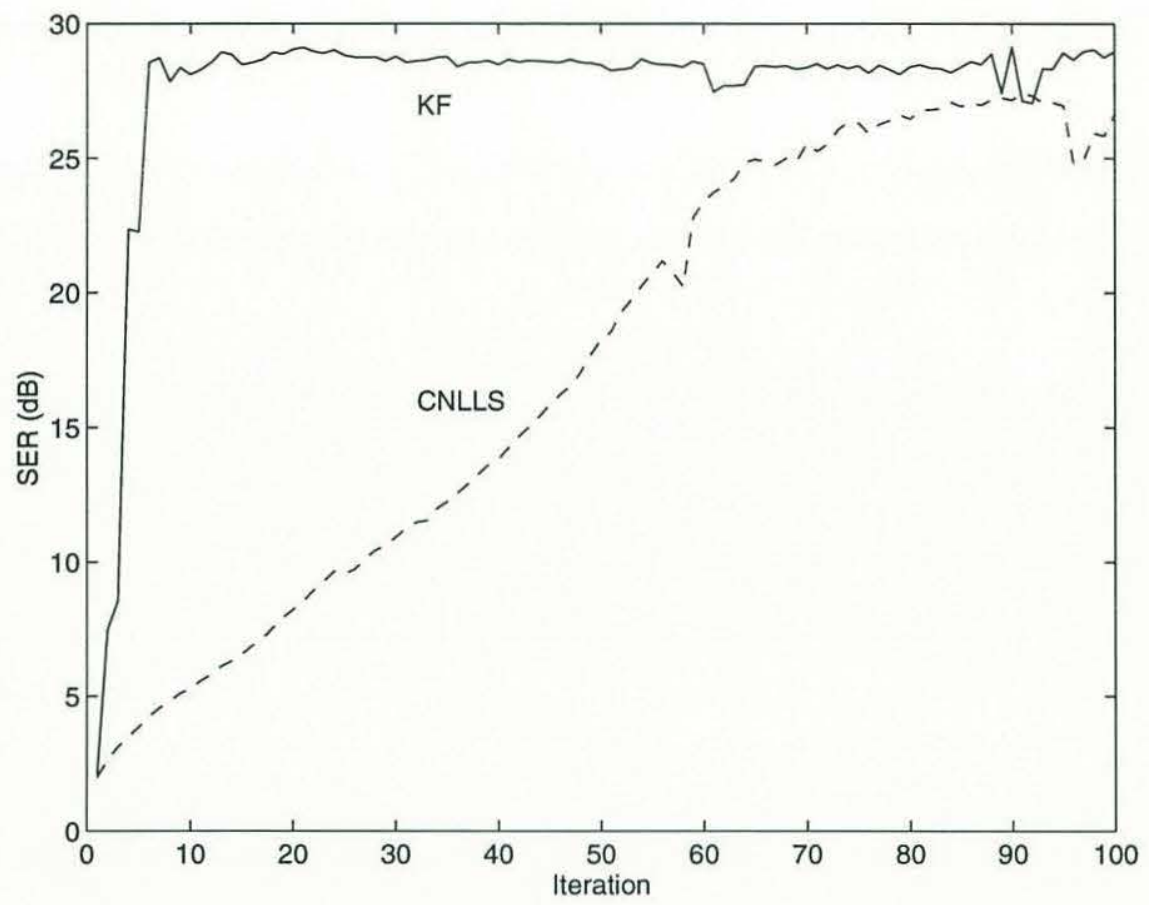

Figure 4-7: SER Performance for System Mode 4 in Time-Invariant Waveguide

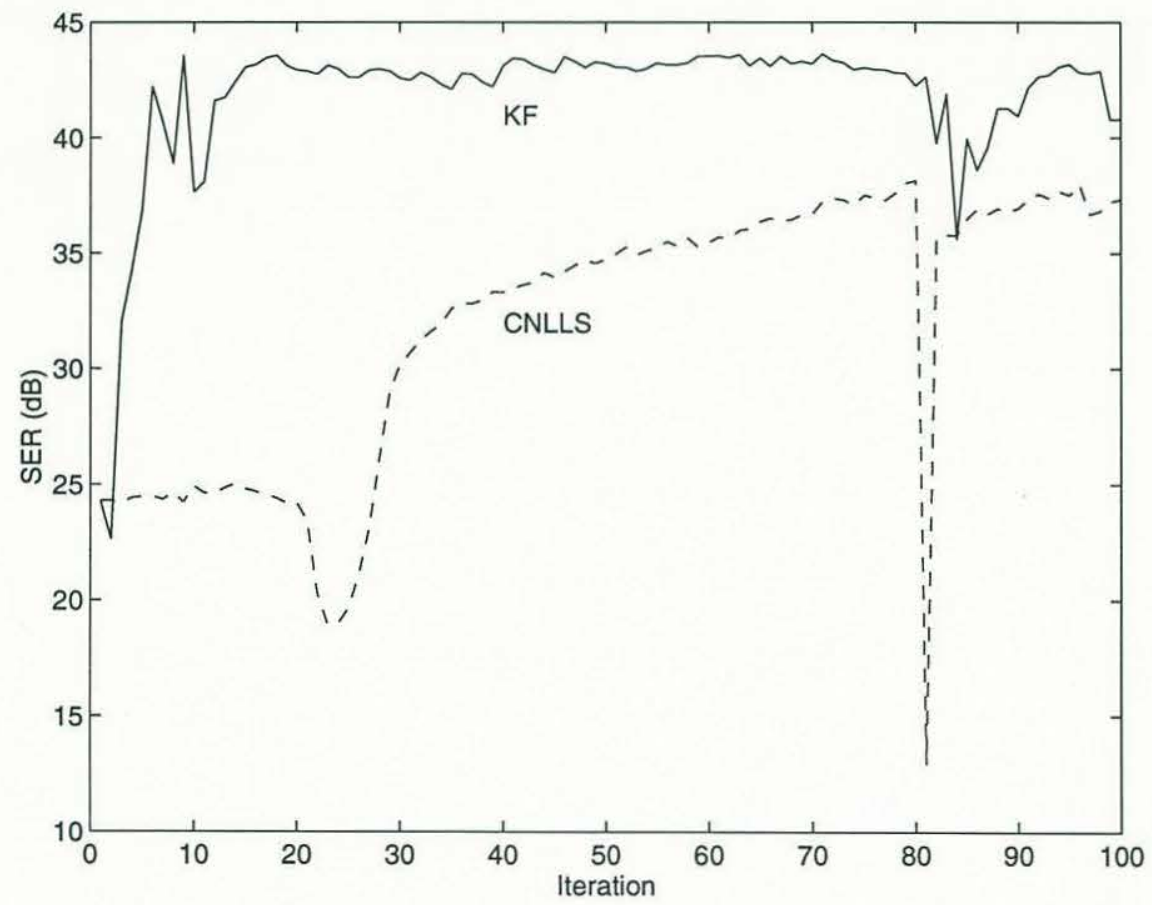

Figure 4-8: SER Performance for Mode $1+0.4 j$ Mode 2 in Time-Invariant Waveguide 
mode one. Figure 4-9 shows the performance for the Kalman filter estimator for various values of $\mathbf{P}_{\mathbf{f}}$ when $\alpha=1.0$. The mean magnitude of the elements of $\mathbf{Q}$ is 0.16 for this experiment. All of the covariance matrices for the experiments in Fig. 4-9 are far below the steady value of most elements of the replica matrix.

With $\alpha=1.0$, the covariance of the Kalman filter estimator will grow in all directions except $\mathbf{w}$. This may explain why the smallest $P_{\mathrm{f}}$ gives the best performance, as the estimator's covariance matrix will grow the slowest for this small innovation covariance. From examining this figure, it is tempting to suggest the high frequency content of the SER increases as $\mathbf{P}_{\mathbf{f}}$ decreases. Plotting the spectra of these curves reveals that the $\mathbf{P}_{\mathbf{f}}=2 \times 10^{-6} \mathbf{I}$ trial has more high frequency content in the SER than the $\mathbf{P}_{\mathbf{f}}=10^{-6} \mathbf{I}$ trial. More importantly, SER is not necessarily a good indicator of the frequency content of the errors. Consider the case when the observed pressure $\mathbf{p}[n]=\mathbf{p}_{d}+\Delta \mathbf{p} e^{j \omega n}$. This would have constant SER plotted against time regardless of the frequency $\omega$ in the error term. Thus, the frequency content of the pressure error may not be reflected in the SER. It is also important to remember that the curves in Fig. 4-9 are from a single trial. The limited time available for experiments precluded running extensive trials in the flume to allow averaging of performance over many trials.

Figure 4-10 plots the performance of the CNLLS estimator with different choices for the forgetting factor $\gamma$. The relatively smallest forgetting factor $(\gamma=0.9$, effective window length $=22$ samples) converges the fastest of the experiments shown, although $\gamma=0.925$ reaches a slightly higher final SER. The strong performance of these relatively short windows is not surprising because the noise level in the flume is so low. Consequently, the channel estimator does not need to average many iterations to minimize the effect of the observation noise. The tighter limit on the inverse of $\boldsymbol{\Phi}[n]$ introduces more bias into the channel estimate than was seen in the simulation results in Chapter 3. This explains why the CNLLS performance does not equal the SER obtained by the Kalman filter in the waveguide, although the 
CNLLS often performed slightly better than the Kalman filter in the simulations of time-invariant ocean channels.

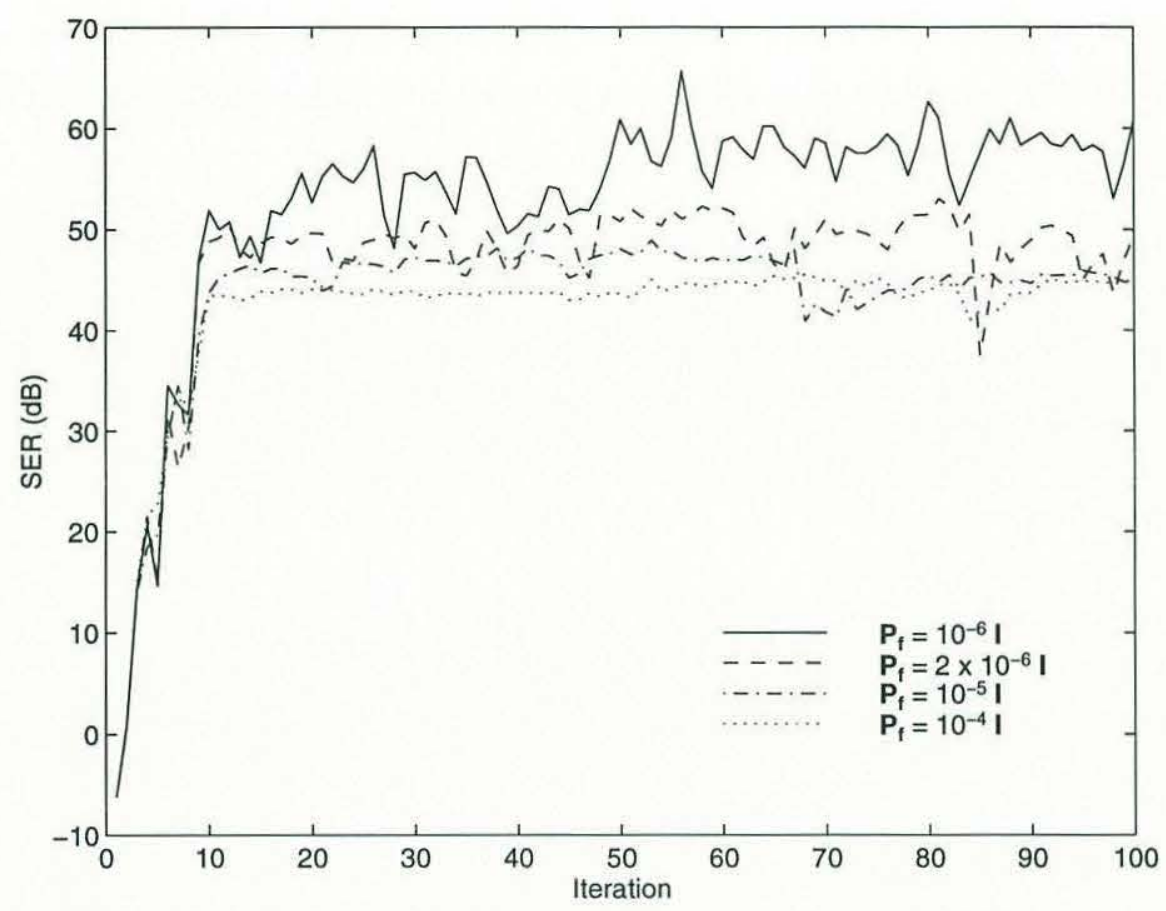

Figure 4-9: Comparison of the SER for System Mode 1 in the Time-Invariant Waveguide for different $\mathbf{P}_{\mathbf{f}}$ in the Kalman Filter Channel Estimator

Figure 4-11 shows the SER of the LMS channel estimator for two different choices of $a$. The algorithm converges faster and to a higher SER for $a=0.5$, although it does not match the performance of the Kalman filter or CNLLS estimators. It is reassuring that these latter estimators do obtain better performance than the LMS for the additional computational complexity they require. Figure 4-12 plots the pressure magnitude profile against depth for all three algorithms, as well as the desired profile for mode one. The Kalman filter and CNLLS estimators generate a pressure profile which is almost indistinguishable from the desired pressure profile. The LMS estimator does not match the desired profile quite as closely, but does still give a good approximation to the profile of system mode one. 


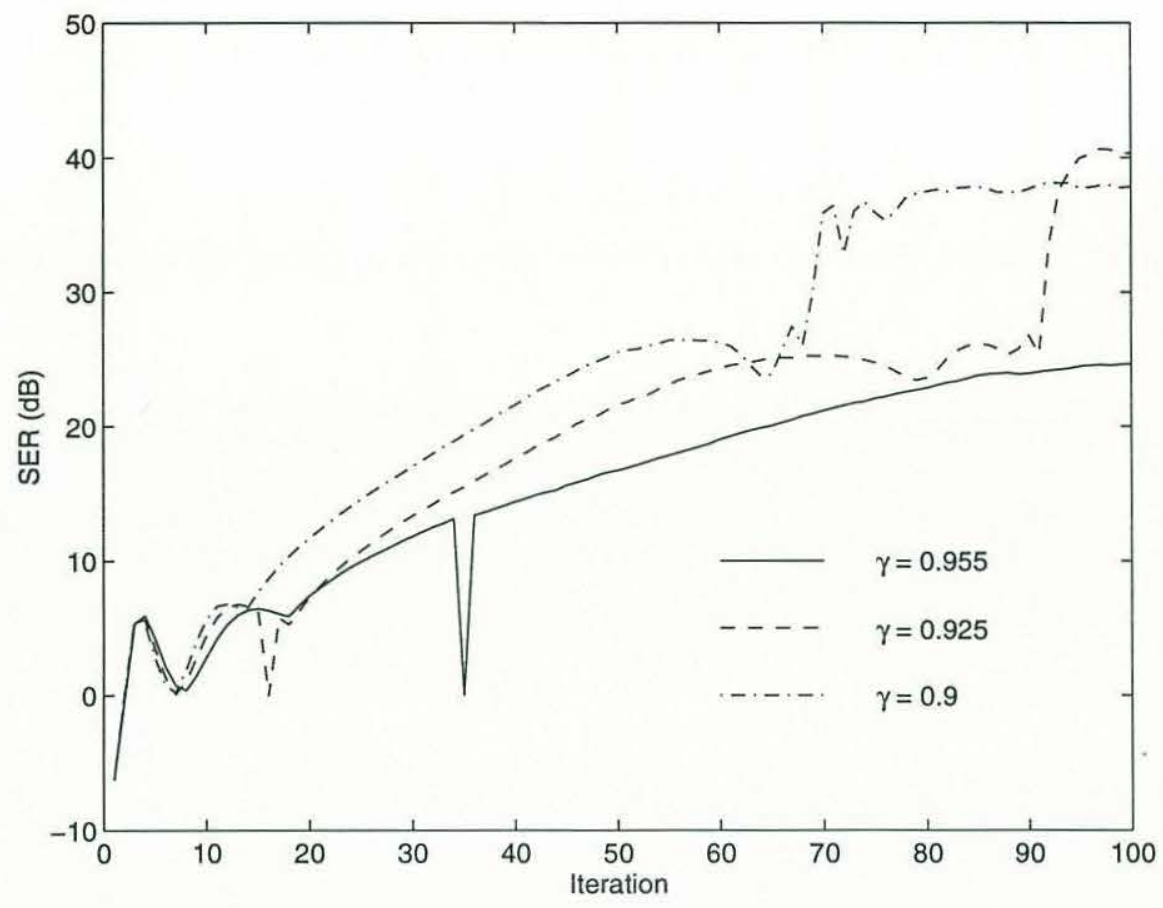

Figure 4-10: Comparison of the SER for System Mode 1 in the Time-Invariant Waveguide for different $\gamma$ in the CNLLS Channel Estimator

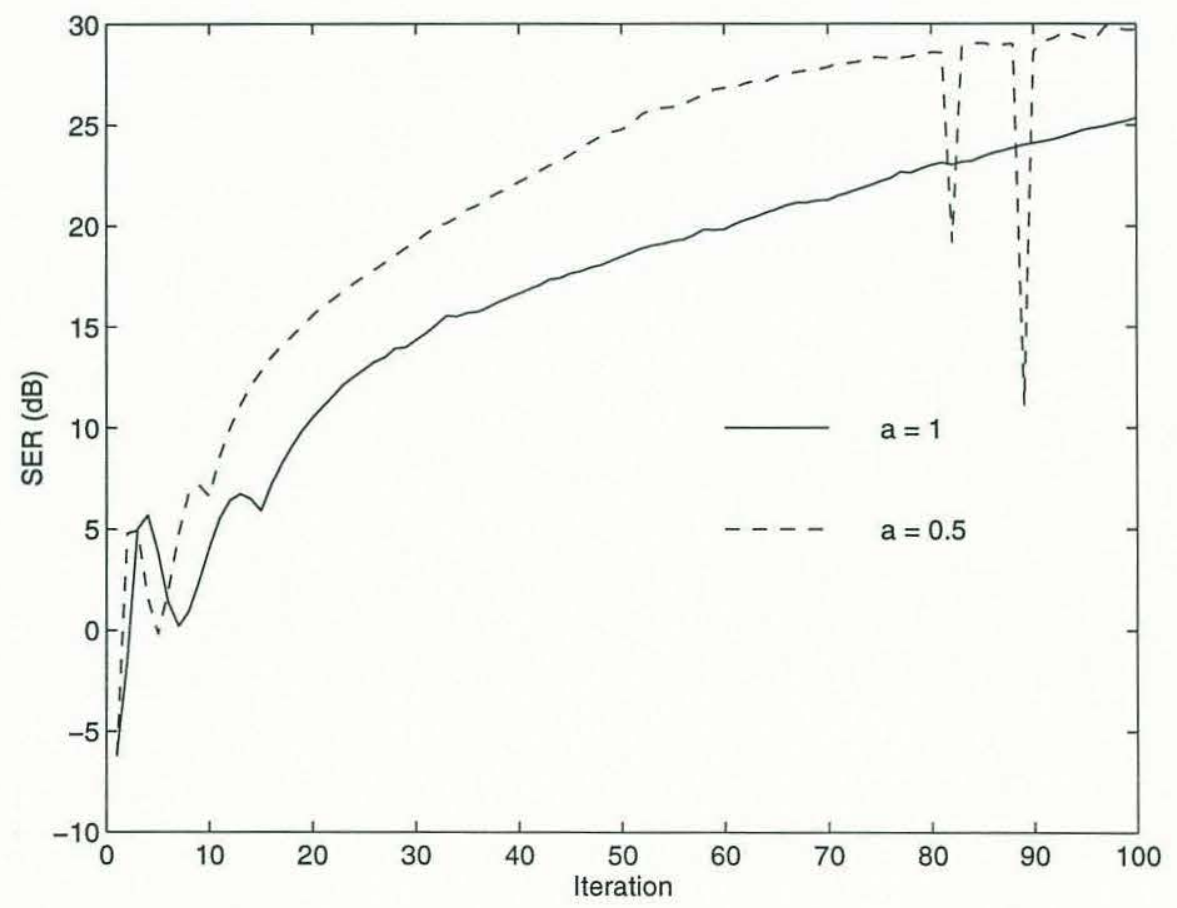

Figure 4-11: Comparison of the SER for System Mode 1 in the Time-Invariant Waveguide for different choices of $a$ in the LMS Channel Estimator 


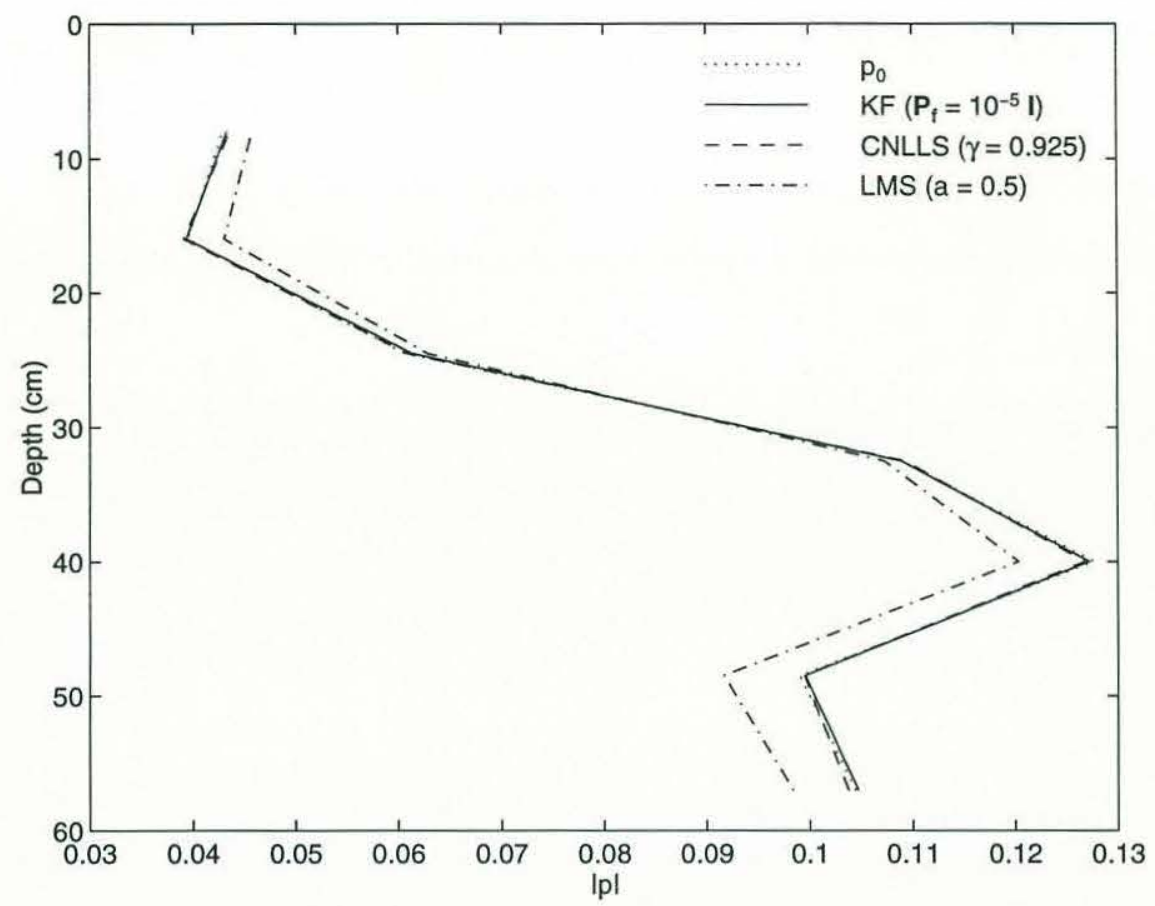

Figure 4-12: Depth Profile of Pressure Magnitude for All Channel Estimators after 100 Iterations

\subsection{Time-Varying Channels}

The experiments presented in this section investigate the transient performance of the feedback control algorithm. These experiments consisted of allowing the algorithm to iterate for a fixed amount of time, then inserting a scatterer to change the acoustic propagation in the waveguide. The scatterer used was a $40 \times 19.5 \times 19.5 \mathrm{~cm}$ cinder block. The block was suspended in a manner allowing it to be consistently placed at $r=3.15 \mathrm{~m}$ in the waveguide such that the bottom of the block was at a depth of roughly $z=25 \mathrm{~cm}$. The dynamic characteristics of the insertion were handcontrolled by a human operator, and were not as consistent between trials as the location was. This difficulty resulted in the two strategies for transient experiments outlined in the following sections.

All the experiments attempted to excite system mode one starting from a corrupted version of the true $\mathbf{Q}$. The Kalman filter estimator used $\mathbf{P}_{\mathbf{f}}=10^{-5} \mathbf{I}$ and 
$\alpha=1.0$, while the CNLLS estimator had $\gamma=0.95$ (effective window length $\approx 45$ iterations) and a condition number limit of 200. The normalized LMS estimator used $a=0.5$. The physical configuration of the waveguide and arrays were the same as described earlier in this chapter.

\subsubsection{Abrupt Channel Change}

These experiments changed the acoustic properties of the waveguide such that from the estimators' point of view the change occurred instantaneously. To implement this, the feedback control algorithm ran for 100 iterations with an open waveguide, then paused. The cinder block was lowered into the waveguide, the water waves excited in the tank were allowed to calm down, and then the algorithm resumed with the same state and desired pressure profile it had when it paused. From the estimators' vantage, the brick appeared instantaneously between iterations without disturbing the water. This experiment was repeated for each of the channel estimators. This method of modifying the acoustic properties of the channel allowed the experiments to focus on the transient behavior of the estimators independently of the settling time of any wave dynamics introduced by the lowering of the cinder block.

Figure 4-13 presents the SER curves for these experiments. The presence of the block does alter the replica matrix and thus the modes of the system. The desired pressure profile at the feedback array is kept unchanged as the first mode of the open waveguide. Projecting this pressure profile on the first three modes of the replica matrix with the block in place reveals more than $99 \%$ of the energy of the desired profile still falls in the span of the first three system modes, i.e.,

$$
\frac{\left\|\left(\mathrm{I}-\sum_{i=1}^{3} \mathrm{u}_{\mathrm{Q} B i} \mathrm{u}_{\mathrm{Q} B i}{ }^{\mathrm{H}}\right) \mathbf{p}_{d}\right\|^{2}}{\left\|\mathrm{p}_{d}\right\|^{2}}<0.01
$$

where $\mathbf{u}_{\mathbf{Q} B i}$ is the $i^{\text {th }}$ column of the replica matrix $\mathbf{Q}$ with the block inserted in 
the waveguide, and $\mathbf{p}_{d}$ is the first mode of the system for the open waveguide. The dotted line at roughly $30 \mathrm{~dB}$ indicates the best possible performance exciting mode one with the block in place. This bound on the SER is down from a theoretical limit of $150 \mathrm{~dB}$ in the absence of noise for the open tank without the block. As the observation noise limits the performance to $45-50 \mathrm{~dB}$ of SER, inserting the block changes the environment from one where the performance is noise-limited to one where the performance is propagation limited by the coupling induced by the block. The Kalman filter estimator adapts to the new channel in roughly 12 iterations. The CNLLS and LMS estimators take longer to converge after the change in the channel. The CNLLS estimator takes about 40 iterations to converge, while the LMS algorithm hasn't converged 100 iterations after the block appears. Both of these convergence characteristics could probably be improved by modifying the estimator parameters: decreasing $\gamma$ for the CNLLS or increasing $\tilde{\mu}$ for the normalized LMS. While these changes would increase the convergence rate, they would also make the estimators more sensitive to observation noise. Decreasing $\gamma$ would decrease the effective window length the CNLLS estimator averages over, while a larger $\tilde{\mu}$ makes the LMS estimator more sensitive to noise even after it has converged. In noisy ocean experiments, this sensitivity would be a more serious issue than in the very quiet and controlled laboratory waveguide setting.

\subsubsection{Gradual Channel Change}

In the experiments described in this section, the feedback control algorithm ran continuously while the cinder block was lowered into the waveguide, rather than pausing as in the experiments described in the previous section. The planned sequence was to allow the algorithm to run for 50 iterations, then gradually insert the block over the next ten to fifteen iterations while trying to minimize the water waves excited in the tank by the disturbance. The execution was not always successful in terms of the timing of the insertion or minimizing the fluid dynamic transient. 


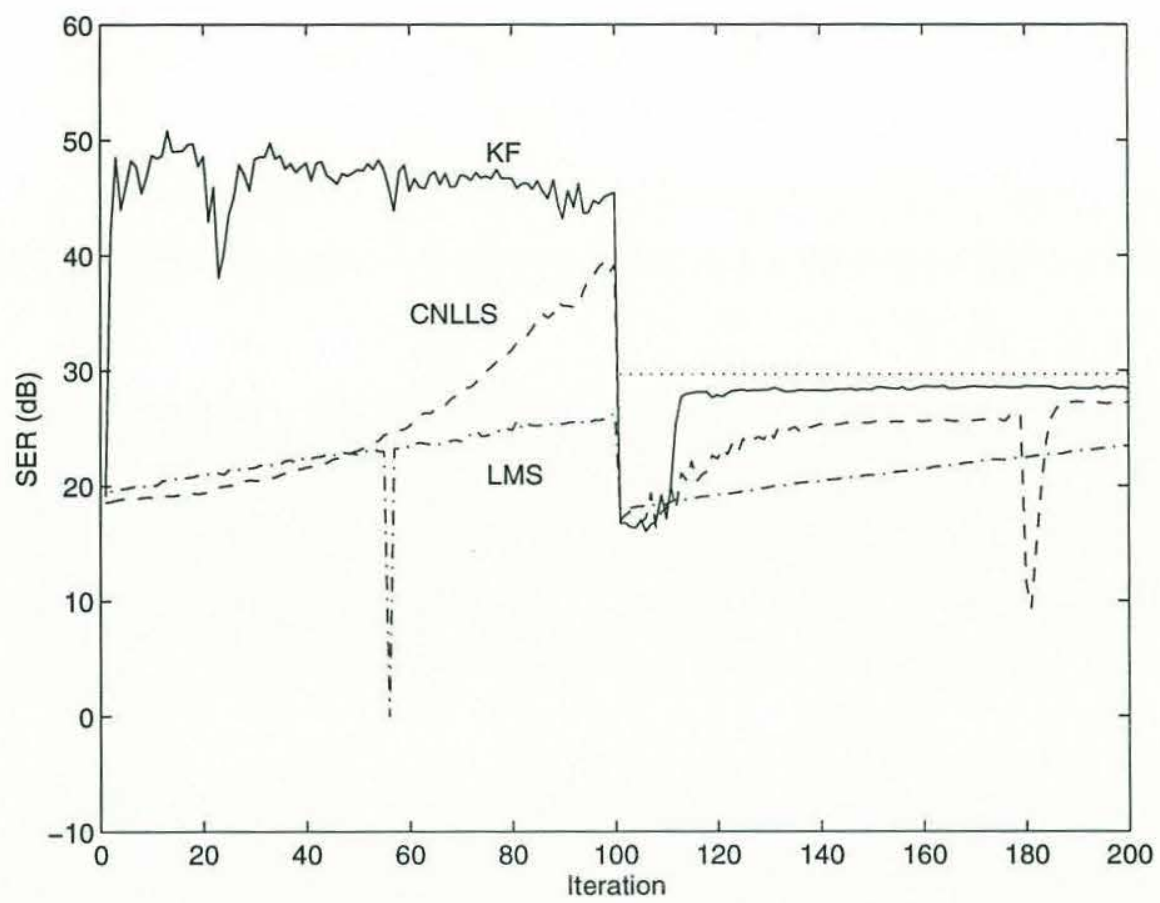

Figure 4-13: Transient Response of Channel Estimators for Abrupt Change in Channel

Figures 4-14 through 4-16 present the results of these experiments. The different line types on each figure represent successive trials of the same experiment. It is not easy to extract the sort of quantitative results obtained in the previous section from these plots. The Kalman filter clearly converges the fastest in these experiments, with the CNLLS and LMS estimators following in that order. Both the Kalman filter and CNLLS estimators come very close to the LS bound on SER with the block in the waveguide. This bound for these trials is not necessarily the same as the one shown in Fig. 4-13 since the waveguide characteristics drift and these trials were performed at a different time and took longer to complete. Consequently, the extent to which $\mathbf{p}_{d}$ fell within the numerical span of $\mathbf{Q}$ varied from trial to trial. This bound only varied by a few $\mathrm{dB}$ over the experiments shown in this section.

The longer convergence times of all the estimators in these experiments when compared to Fig 4-13 could be due to a combination of several factors. The acoustic 
properties of the waveguide are changing every iteration for roughly a dozen iterations due to the lowering of the block into the waveguide. In addition, the water waves excited by inserting the block will also affect the acoustic propagation. Even a very gentle touch lowering the block into the channel excited water waves that took several iterations to settle.

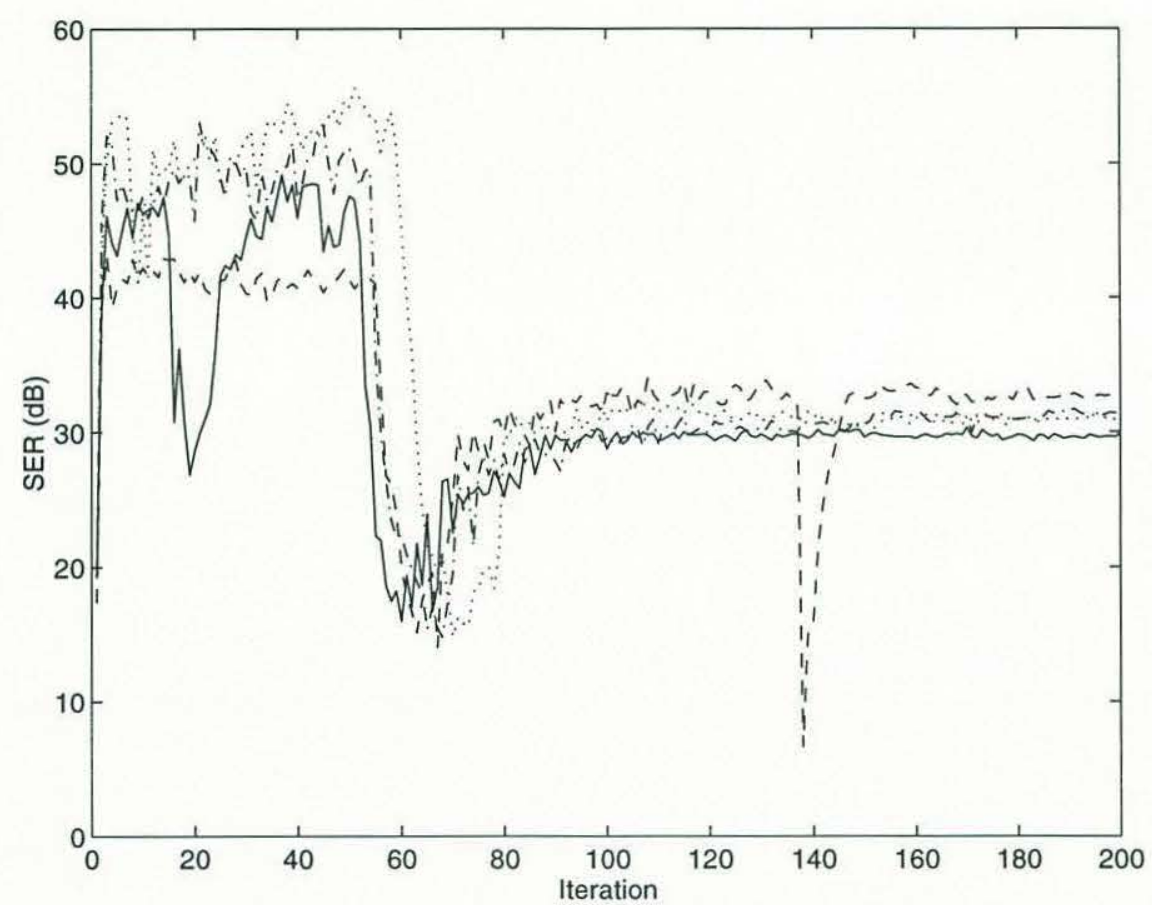

Figure 4-14: Transient Response of Kalman Filter Channel Estimator for Gradual Change in the Waveguide

A rough quantitative estimate of the increase in convergence time can be obtained by starting from the observation that in most of the trials in these experiments the block was completely lowered by iteration 65. Looking closely at Fig. 4-14 reveals the Kalman filter estimator generally converged within 25 iterations after the block was in place. This was about twice as long as the convergence time observed in Fig. 4-13. A similar examination of Fig. 4-15 reveals an increase of convergence time to roughly 70 iterations, rather than the 40 iterations observed in Fig. 4-13. The LMS algorithm never completely converged in either set of experiments, making it impossible to 


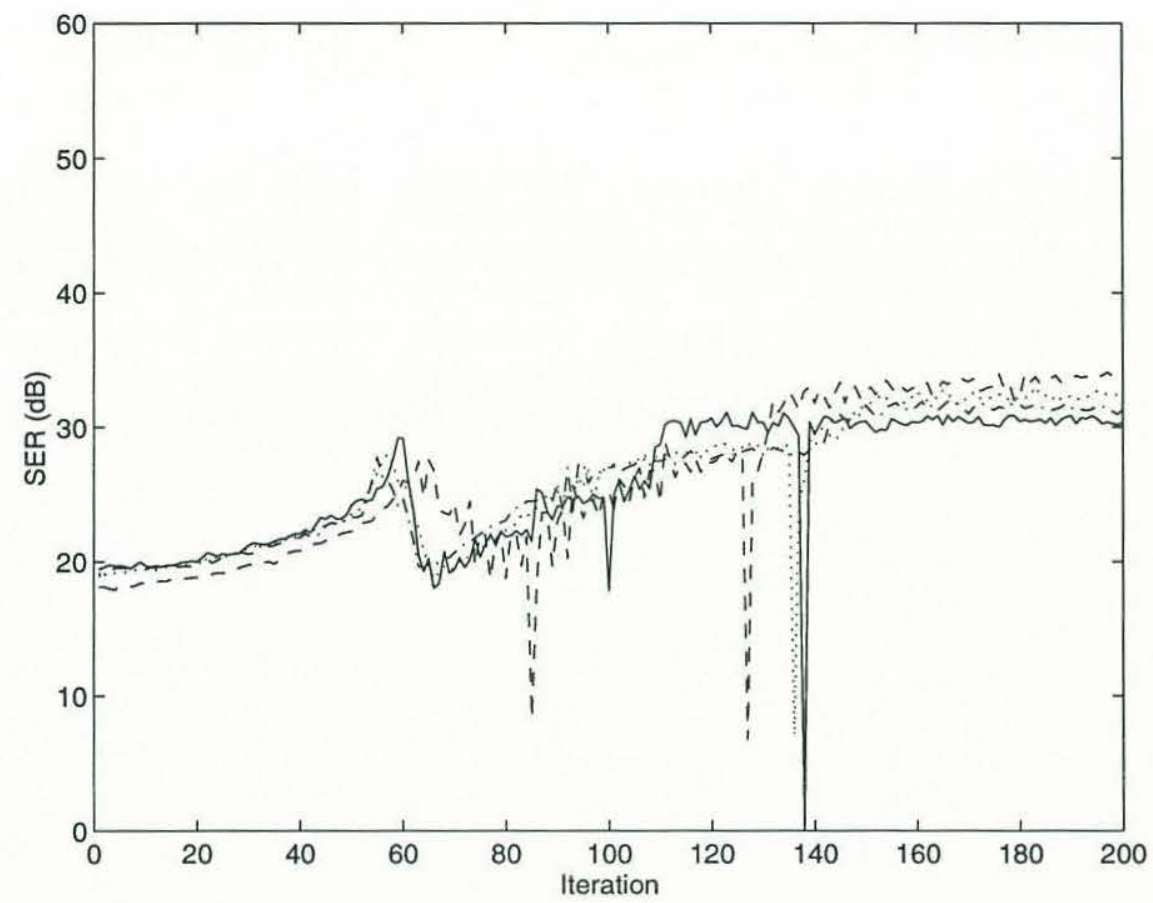

Figure 4-15: Transient Response of CNLLS Channel Estimator for Gradual Change in the Waveguide

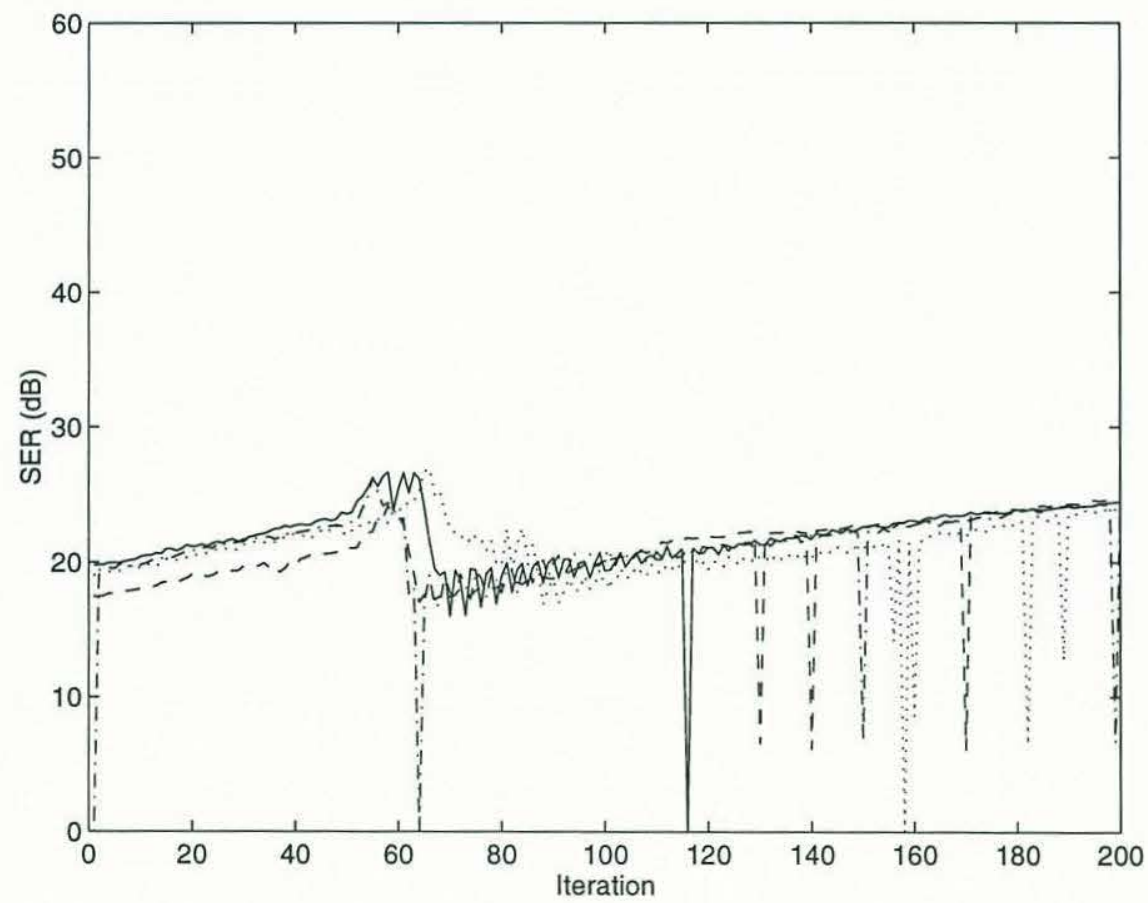

Figure 4-16: Transient Response of LMS Channel Estimator for Gradual Change in the Waveguide 
make a similar comparison for this estimator. Comparing these transient response times to those seen with the abrupt channel change, one qualitative conclusion that can be made is water dynamics slow the convergence of the estimators. While the insertion of the block was not perfectly consistent for each trial, the water waves were excited in roughly the same way. The varying transient response times among the estimators indicate that the estimators experience differing levels of difficulty tracking the water dynamics. If the dynamics were slow enough that the estimators could perfectly track the changes in $\mathrm{Q}$, all of the transient times of the estimators would be the settling time of the water dynamics. The variation of the response times in Figs. 4-14 through 4-16 confirm the estimators are unable to keep up with the changes in $\mathbf{Q}$ due to the hydrodynamics. The Kalman filter does not assume the replica matrix dynamics are a stationary process, so it is not surprising that it adapts most quickly. In fact, the transient time for the Kalman filter might be taken as a rough estimate of the settling time for the dynamics of the water waves. The CNLLS and LMS estimators assume $\mathbf{Q}$ is stationary, so they won't be able to make substantial progress converging on the new $\mathrm{Q}$ with the block in place until the non-stationarity introduced by the water waves dies out. For the CNLLS estimator, the increase in convergence time from the abrupt channel change experiment should reflect the settling time for the water dynamics. The increase in convergence time of roughly 30 iterations is commensurate with the estimate of the time constant for the hydrodynamics obtained from the Kalman filter transient. The duration of the ripples in the LMS SER in Fig. 4-16 also supports this estimate of the hydrodynamics time constant. As noted in the discussion of Fig 4-9, time characteristics of the SER do not necessarily reveal all the underlying dynamics of the error, but here the SER curve for the LMS estimator does corroborate the estimated settling time for the water waves obtained from the CNLLS and Kalman filter SER curves.

The estimators' difficulty tracking the flume water dynamics are not necessarily cause for concern in the ocean environment. Visual observation indicated the waves 
excited by lowering the block propagated the $8 \mathrm{~m}$ from the block to the feedback array in less than 3 seconds. Scaling up by a factor of 20 for a $400 \mathrm{~Hz}$ ocean experiment, this indicates that in an ocean experiment the estimators could not track water dynamics propagating $26 \mathrm{~m} / \mathrm{s}$, an excessive velocity for ocean phenomena. The simulations of the solitary wave in Section 3.4 suggests the Kalman filter can track ocean processes propagating at least as fast as $1 \mathrm{~m} / \mathrm{s}$, which is a generous estimate for the group velocity of these waves.

\subsection{Summary}

This chapter presented a sequence of experiments using the proposed feedback control algorithm in a laboratory waveguide. These experiments demonstrate that the algorithm performs well in time-invariant channels when implemented on real-time signal processing hardware connected to real sources and hydrophones. This environment provided a very useful test bed for verifying the algorithm was feasible and making some preliminary investigations of its transient behavior. More detailed investigations of the tracking performance of the estimators would require a well-controlled means of introducing a fluid dynamic disturbance of the appropriate bandwidth in the waveguide. The results obtained in the experiments described in this chapter are encouraging for the success of an ocean experiment. In addition, the experiments provided some valuable experience for the deployment and debugging of a real-time system implementing the feedback control algorithm. 


\section{Chapter 5}

\section{Conclusions and Future Directions}

The work presented in this thesis develops an adaptive feedback controller to excite a single mode in the shallow water channel. No mid-frequency (circa $400 \mathrm{~Hz}$ ) ocean experiment has successfully generated a single mode in this environment. A single mode source in this frequency range would be very useful for studying oceanographic processes in the coastal environment. The control algorithm incorporates elements of control theory, adaptive estimation, array processing and underwater acoustics. The controller iterates between choosing the source array weight vector, and updating its estimate of the replica matrix between the source and feedback arrays. Three channel estimators are developed based on different models for the properties and statistics of the replica matrix.

The work leading to the control algorithm also yielded two additional results. The first is a formulation of the maximum a posteriori (MAP) mode filter. This filter segues between the pseudo-inverse and sampled mode shape filters as the hydrophone array aperture decreases. This result provides a theoretical basis for an earlier ad hoc "dropped eigenvalue" mode filter proposed by Yang [41]. The MAP mode filter also has an appealing interpretation as a generalization of the discrete spatial Wiener filter. The investigation of controllability and observability provides a cohesive framework for examining numerical issues germane to a practical exper- 
iment. Specifically, criteria are given for determining when a desired mode can be excited and observed given proposed array configurations, observation noise characteristics, source power limitations and approximate acoustic attenuation in range.

A series of simulations presented in Chapter 3 investigate the performance of the proposed algorithm in several realistic shallow water environments: a range-invariant waveguide, a rock outcropping, a downsloping wedge, and a propagating solitary internal wave. The performance of the various channel estimators in each environment are compared to each other and the performance of an open loop controller. The Kalman filter and CNLLS estimators both generated high fidelity modes in these simulations, with the latter usually performing slightly better.

The proposed algorithm was also evaluated in a series of laboratory waveguide experiments. The algorithm successfully excited several different modes of the system. The Kalman filter channel estimator proved more robust to the computational limitations of the real-time DSP hardware in these experiments. The waveguide experiments also provided some preliminary data on the transient response of the channel estimators. The Kalman filter also performed better than the other estimators in the transient experiments. The success of the algorithm in the laboratory waveguide is encouraging for the prospects of an ocean experiment.

Several aspects of the single mode excitation problem present intriguing directions for future research. One possible improvement in the feedback control algorithm would be in the weight vector selection step. Rather than choosing the source array weight vector $\mathbf{w}[n]$ to minimize the squared error between the predicted pressure $\hat{\mathbf{Q}}[n] \mathbf{w}[n]$ and desired pressure vector $\mathbf{p}_{d}, \mathbf{w}[n]$ could be chosen to minimize the ratio of the energy in all modes to the energy in the desired mode. In the limit with only mode $m_{0}$ excited, this ratio would be 1 . More precisely, the optimization problem the new weight selection step would solve is

$$
\mathbf{w}[n]=\arg \min _{\mathbf{w}} \frac{\mathbf{w}^{\mathrm{H}} \hat{\mathbf{B}}^{\mathrm{H}}[n] \hat{\mathbf{B}}[n] \mathbf{w}}{\mathbf{w}^{\mathrm{H}} \hat{\mathbf{b}}_{m_{0}}{ }^{\mathrm{H}}[n] \hat{\mathbf{b}}_{m_{0}}[n] \mathbf{w}},
$$


where $\hat{\mathbf{B}}[n]$ is an estimate of the source weight to mode coefficient transfer matrix from Eq. 1.35 and $\hat{\mathbf{b}}_{m_{0}}[n]$ is the $m_{0}^{\text {th }}$ row of that estimate. As formulated in Eq. 5.1, the optimization does not have a unique solution, but by introducing an additional constraint, a unique solution can be obtained. The constrained optimization is

$$
\mathbf{w}[n]=\arg \min _{\mathbf{w}} \mathbf{w}^{\mathrm{H}} \hat{\mathbf{B}}^{\mathrm{H}}[n] \hat{\mathbf{B}}[n] \mathbf{w} \text { subject to } \hat{\mathbf{b}}_{m_{0}}[n] \mathbf{w}=1 .
$$

and it is clear any solution to this optimization will also be a solution to Eq. 5.1. The constrained problem in Eq. 5.2 has the same form as the Minimum Variance Spectral Estimator [66], [40]. The solution to this optimization can be written in closed form:

$$
\mathbf{w}[n]=\frac{\left(\hat{\mathbf{B}}^{\mathrm{H}}[n] \hat{\mathbf{B}}[n]\right)^{-1} \hat{\mathbf{b}}_{m_{0}}{ }^{\mathrm{H}}[n]}{\hat{\mathbf{b}}_{m_{0}}[n]\left(\hat{\mathbf{B}}^{\mathrm{H}}[n] \hat{\mathbf{B}}[n]\right)^{-1} \hat{\mathbf{b}}_{m_{0}}{ }^{\mathrm{H}}[n]} .
$$

The performance of this weight selection criteria would need to be evaluated in simulations and laboratory waveguide experiments and compared against the performance of the least-squares criteria used in the thesis.

The proposed algorithm uses a very basic model for the acoustic propagation in the feedback volume and also a simple feedback control algorithm. The simulations and laboratory experiments indicate these straightforward approaches can successfully excite a single mode. This success encourages further investigations incorporating more sophisticated control algorithms and ocean models to see if these advances can provide even better performance. Specifically, the field of robust control contains many important results that may provide additional insight into the single mode control problem [49], [50], [51]. In pursuing these advances, a balance must be made between improved performance exciting a mode and greater sensitivity to mismatch errors in either the ocean acoustics or estimated plant. While the algorithm proposed here is very modest in its technical scope, it is flexible in responding to a wide range of ocean environments. Any more sophisticated control algorithm will want to preserve this robustness as much as possible. 
Another possible improvement would be a more aggressive temporal sampling strategy for the feedback volume. The iteration time could be reduced from the propagation time of the slowest mode between the source and feedback arrays to the difference between the travel times of the fastest and slowest modes. This would require accurate estimates of the mode group velocities. These estimates could be obtained numerically from the sound speed profiles at the source and feedback arrays [32] and if the estimates are sufficiently accurate and reliable, several packets of modes could be en route between the source and feedback arrays simultaneously. This decrease in the iteration time for the control algorithm would allow the channel estimator to track a higher bandwidth of oceanographic processes. For the ranges and environments examined in this thesis, the conservative iteration interval set by the propagation time of the slowest mode sufficiently samples the most important processes, so the incremental gains obtained by decreasing the iteration time do not merit the significantly increased risk of poor performance if the estimated group velocities are incorrect. Inaccurate group velocity estimates could cause mode packets that are no longer temporally distinct at the feedback array. Future deployments of the algorithm may occur in environments with ocean processes operating on shorter time scales, or with a greater distance between the source and feedback arrays. In such scenarios, the benefits of reducing the iteration time might merit the additional complexity and risk associated with this strategy. A specific instance where this could prove valuable would be scenarios where surface wave processes have a significant effect on the mode propagation. Surface waves have much shorter time scales than most internal ocean processes and reducing the iteration time of the control algorithm could prove crucial to allowing the estimators to track these processes.

The single mode source provided by the feedback control algorithm can be used for other acoustic measurements besides estimating the horizontal wavenumber spectra of an internal wave packet. When the propagation in the observation volume is well-modeled by a discrete set of propagating modes, the mode coupling over that 
volume gives a simple parametric model for predicting the propagation through that volume. Long-term measurements of these coupling statistics could provide useful measurements of the dynamics of acoustic propagation in coastal regions which could be exploited by more sophisticated ocean acoustics signal processing algorithms. Currently, very little data is available about the statistics and dynamic behavior of propagation in these regions.

The feedback control algorithm may have other oceanographic applications besides a single mode source. Gingras [5] proposed a single mode source as a method of minimizing environmental backscatter for an active sonar system. While a single propagating mode is a useful conceptual approach to visualize which portions of the water column would be illuminated by such a system, such a pressure profile may not be the optimal transmitted pressure field to minimize backscatter from the bathymetry. The feedback control algorithm could adapt the source array to minimize the backscattered energy in the absence of targets.

Another possible but highly speculative use for the feedback control algorithm would be in a communication scenario. Different messages could be modulated onto distinct modes for a communication network. This would allow simultaneous communication at the same frequency by exploiting the modal diversity of the environment. Any communication system proposed utilizing this modulation would have to address many issues such as mode coupling due to range-inhomogeneities in the water column or bottom bathymetry between the feedback array and receiver. The concept is attractive and bears closer examination.

The ideas presented in this section are intriguing as extensions or improvements on the algorithm presented in the thesis. At the current time, the most pressing future work to be done is an actual ocean test of the algorithm proposed. Significant engineering challenges such as the radio telemetry link from the feedback array to the source ship and continuous mode shape estimation at the feedback array location must still be addressed. The simulations and laboratory experiments indicate that 
the feedback control algorithm should be capable of exciting a single mode. If this experiment is successful, more advanced uses can be explored. If the trial reveals unexpected shortcomings or difficulties with the feedback control algorithm, these problems can be addressed. Without such an experiment, any future work performed with the algorithm must be viewed as speculative at best. 


\section{Appendix A}

\section{Transient Response of the CNLLS}

\section{Estimator}

Section 2.3 describes the CNLLS estimator and notes that it is important to limit the condition number of $\Phi^{-1}$ at each iteration and not the condition number of $\Phi$ propagated by the algorithm. Specifically, the estimator may respond more slowly to abrupt changes in the desired mode or the channel if the condition number of $\Phi$ is limited. To understand why, consider the simplified case where $\mathbf{w}[n]=\mathbf{w}_{0}$ for $n=1, \ldots, 2 \eta$, where $\eta$ is the limit on the condition number. The eigenvalue decomposition of $\boldsymbol{\Phi}[2 \eta]$ can be written

$$
\Phi[2 \eta]=\mathrm{V}_{\boldsymbol{\Phi}}\left[\begin{array}{cccc}
2 \eta & 0 & \cdots & 0 \\
0 & 0 & \ddots & \vdots \\
\vdots & \ddots & \ddots & 0 \\
0 & \cdots & 0 & 0
\end{array}\right] \mathrm{V}_{\boldsymbol{\Phi}}{ }^{\mathrm{H}}
$$

where

$$
\mathrm{V}_{\mathbf{\Phi}}=\left[\mathrm{w}_{0}\left|\mathbf{v}_{2}\right| \cdots \mid \mathbf{v}_{L}\right] .
$$


Suppose the desired mode changes abruptly at $n=2 \eta$ such that $\mathbf{w}[n]$ is now orthogonal to $\mathbf{w}_{0}$ for $n>2 \eta$. Note that if $\mathbf{w}[n]$ is orthogonal to $\mathbf{w}_{0}$, we may assume $\mathbf{w}[n]=\mathbf{v}_{2}$ without loss of generality, since we can pick the $\mathbf{v}_{i}$ to be any orthonormal basis of orthogonal complement of $\mathbf{w}_{0}$. Moreover, assume $\hat{\mathbf{Q}}[n]$ has errors that were orthogonal to $\mathbf{w}_{0}$ but are not orthogonal to $\mathbf{v}_{2}$, i.e., $(\hat{\mathbf{Q}}[n]-\mathbf{Q}[n]) \mathbf{v}_{2} \neq 0$. The change of $\mathbf{w}[n]$ to $\mathbf{v}_{2}$ will cause $\hat{\mathbf{Q}}[n]$ to converge to a more accurate estimate of $\mathbf{Q}[n]$ such that $(\hat{\mathbf{Q}}[n]-\mathbf{Q}[n]) \mathbf{v}_{2}=\mathbf{0}$.

The rate of this convergence depends on which of $\Phi$ or $\Phi^{-1}$ has its condition number limited. From Eqs. 2.21 and 2.22, the correction to $\hat{\mathbf{Q}}[n]$ is $\mathbf{a}[n] \mathbf{k}^{\mathrm{H}}[n]$, where $\mathbf{a}[n]$ is $(\mathbf{p}[n]-\hat{\mathbf{Q}}[n] \mathbf{w}[n])=(\mathbf{Q}[n]-\hat{\mathbf{Q}}[n]) \mathbf{w}[n]$ and $\mathbf{k}[n]=\mathbf{\Phi}^{-1}[n] \mathbf{w}[n]$.

Let $\mathbf{k}_{1}[n]$ be the gain vector when $\boldsymbol{\Phi}[n]$ is propagated with its condition number limited, and let $\mathbf{k}_{2}[n]$ be the gain vector when $\boldsymbol{\Phi}[n]$ propagates without any limit on its condition number, but the condition number is limited at each iteration when $\Phi_{C L}^{-1}$ is computed per Eq. 2.25. The notation $(\cdot)_{C L}$ denotes the operation of limiting the condition number of its argument to be $\eta$ while leaving the first eigenvalue unchanged. Then

$$
\begin{aligned}
& \mathbf{k}_{1}[2 \eta+1]=\left((\Phi)_{C L}[2 \eta+1]\right)^{-1} \mathbf{w}[2 \eta+1] \\
& =\mathrm{V}_{\Phi}\left[\begin{array}{ccccc}
2 \eta & & 0 & \cdots & 0 \\
& 3 & & \ddots & \vdots \\
& & 2 & & 0 \\
\vdots & \ddots & & \ddots & \\
0 & \cdots & 0 & & 2
\end{array}\right]^{-1} \quad \mathrm{~V}_{\Phi}{ }^{\mathrm{H}} \mathbf{v}_{2} \\
& =\mathbf{v}_{2} / 3 \text {. }
\end{aligned}
$$


Contrastingly, for the CNLLS estimator given in Sec. 2.3,

$$
\begin{aligned}
& \mathbf{k}_{2}[2 \eta+1]=\left(\Phi^{-1}\right)_{C L}[2 \eta+1] \mathbf{w}[2 \eta+1] \\
& \begin{aligned}
= & \mathbf{V}_{\boldsymbol{\Phi}}\left(\left[\begin{array}{ccccc}
2 \eta & & 0 & \cdots & 0 \\
& 1 & & \ddots & \vdots \\
0 & & 0 & & 0 \\
\vdots & \ddots & & \ddots & \\
0 & \cdots & 0 & & 0
\end{array}\right]_{C L}^{-1}\right)_{\mathbf{V}^{H} \mathbf{V}_{2}} \\
= & \mathbf{v}_{2} / 2
\end{aligned}
\end{aligned}
$$

The gain vector $\mathbf{k}[n]$ has the same direction in each case, but takes a larger step for the case where the condition number is propagated without limiting, then limited after inversion. If the correlation matrix $\Phi$ is propagated with condition number limiting, the gain of the correction is reduced even the first step after $\mathbf{w}[n]$ changes. In fact, if $\mathbf{k}_{2}[2 \eta+2]$ will still be $\mathbf{v}_{2} / 2$, while $\mathbf{k}_{1}[2 \eta+2]=\mathbf{v}_{2} / 4$. As long as $\mathbf{w}[n]=\mathbf{v}_{2}$, $\mathbf{k}_{1}[n]$ and $\mathbf{k}_{2}[n]$ will have the same direction, but $\left\|\mathbf{k}_{1}[n]\right\|<\left\|\mathbf{k}_{2}[n]\right\|$, so $\mathbf{k}_{2}[n]$ will have a faster transient response.

The scenario here simplifies the case in several aspects, but the underlying results are still valid: propagating $\Phi[n]$ with its condition number unlimited then limiting the condition number at the inversion for each iteration gives better transient performance than propagating a $\Phi[n]$ whose condition number is limited. 


\section{Bibliography}

[1] Clarence S. Clay and Herman Medwin. Acoustical Oceanography: Principles and Applications. Ocean Engineering. John Wiley \& Sons, New York, NY, 1977.

[2] C. L. Pekeris. Theory of propagation of explosive sound in shallow water. The Geological Society of America Memoirs, 27, October 1948.

[3] D. Peregrym. An investigation of shallow water mode coupling effects during single mode transmission. Master's thesis, MIT/WHOI Joint Program Oceanography and Oceanographic Engineering, August 1994.

[4] J. Zhou, X. Zhang, and P. Rogers. Resonant interaction of sound wave with internal solitons in the coastal zone. Journal of the Acoustical Society of America, 90(4):2042-2054, October 1991.

[5] D. F. Gingras. Shallow-water backscatter reduction through selective mode excitation. Journal of the Acoustical Society of America, 96(5):3288, November 1994.

[6] W. A. Kuperman and F. Ingenito. Spatial correlation of surface generated noise in a stratified ocean. Journal of the Acoustical Society of America, 67(6):19881996, June 1980.

[7] R. M. Hamson. The theoretical responses of vertical and horizontal line arrays to wind-induced noise in shallow water. Journal of the Acoustical Society of America, 78(5):1702-1712, November 1985. 
[8] Raymond H. Ferris. Comparison of measured and calculated normal-mode amplitude functions for acoustic waves in shallow water. Journal of the Acoustical Society of America, 52(3):981-988, September 1972.

[9] C. T. Tindle, K. M. Guthrie, G. E. J. Bold, M. D. Johns, D. Jones, K. O. Dixon, and T. G. Birdsall. Measurements of the frequency dependence of normal modes. Journal of the Acoustical Society of America, 64(4):1178-1185, October 1978.

[10] Homer P. Bucker. Normal-mode sound propagation in shallow water. Journal of the Acoustical Society of America, 36:251-258, 1964.

[11] R. H. Ferris, F. Ingenito, and A. L. Faber. Experimental separation and identification of acoustic normal modes in shallow water. Technical Report 7174, Naval Research Laboratory, Washington, DC, October 1970.

[12] R. R. Leitch and M. O. Tokhi. Active noise control systems. IEE Procedings, 134(6):525-546, June 1987.

[13] B. Widrow and S. Stearns. Adaptive Signal Processing. Prentice Hall Signal Processing Series. Prentice Hall, Englewood Cliffs, NJ, 1985.

[14] Kumpati S. Narendra and Anuradha M. Annaswamy. Stable Adaptive Systems. Information and System Sciences Series. Prentice Hall, Englewood Cliffs, NJ, 1989.

[15] C. S. Clay and K. Huang. Single mode transmission and acoustic backscattering measurements in a laboratory waveguide. Journal of the Acoustical Society of America, 67(3):792-794, March 1980.

[16] C. Gazanhes and J. L. Garnier. Experiments on single mode excitation in shallow water propagation. Journal of the Acoustical Society of America, 69(4):963969, April 1981. 
[17] C. S. Clay. Waveguides, arrays, and filters. Geophysics, 31:501-505, 1966.

[18] R. P. Porter, M. Siderius, W. L. J. Fox, and D. Rouseff. Acoustic prediction in shallow water from sparse data. Journal of the Acoustical Society of America, 95(5):2926, May 1994.

[19] O. Lee. Effect of an internal wave on sound in the ocean. Journal of the Acoustical Society of America, 33(5):677-681, May 1961.

[20] J. C. Preisig and T. Duda. Coupled acousctic mode propagation through continental shelf internal solitary waves. IEEE Journal of Oceanic Engineering, 22(3), To Appear July 1997. Special Issue on Shallow Water Acoustics, Geophysics and Oceanography.

[21] M. D. Collins. FEPE User's Guide. Technical Note 365, Naval Ocean Research and Development Activity, Stennis Space Center, Mississippi, 39529-5004, October 1988.

[22] George V. Frisk. Ocean and Seabed Acoustics: A Theory of Wave Propagation. Prentice Hall, Englewood Cliffs, NJ, 1994.

[23] L. M. Brekhovshikh and Yu. P. Lysanov. Fundamentals of Ocean Acoustics. Springer Series on Wave Phenomena. Springer-Verlag, Berlin, second edition, 1991.

[24] Y. Desaubies. A uniformly valid solution for acoustic normal mode propagation in a range varying ocean. Journal of the Acoustical Society of America, 76(2):624-626, August 1984.

[25] Y. Desaubies, C.-S. Chiu, and J. H. Miller. Acoustic mode propagation in a range-dependent ocean. Journal of the Acoustical Society of America, 80(4):1148-1160, October 1986. 
[26] Ewing and Worzel. Explosion sounds in shallow water. The Geological Society of America Memoirs, 27, October 1948.

[27] Homer P. Bucker. Sound propagation in a channel with lossy boundaries. Journal of the Acoustical Society of America, 48:1187-1194, 1970.

[28] M. Buckingham. Acoustic propagation in a wedge-shaped ocean. In Acoustics of the Sea Bed, pages 251-259. Bath Univ. Press, Bath, U. K., 1983.

[29] M. Buckingham. Theory of three-dimensional acoustic propagation in a wedgelike ocean with a penetrable bottom. Journal of the Acoustical Society of America, 82(1):198-210, July 1987.

[30] C. T. Tindle, H. Hobaek, and T. G. Muir. Downslope propagation of normal modes in a shallow water wedge. Journal of the Acoustical Society of America, 81(2):275-286, February 1987.

[31] C. T. Tindle, H. Hobaek, and T. G. Muir. Normal mode filtering for downslope propagation in a shallow water wedge. Journal of the Acoustical Society of America, 81(2):287-294, February 1987.

[32] Finn B. Jensen, William A. Kuperman, Michael B. Porter, and Henrik Schmidt. Computational Ocean Acoustics. AIP Series in Modern Acoustics and Signal Processing. AIP Press, Woodbury, NY, 1994.

[33] S. L. Campbell and C. D. Meyer Jr. Generalized Inverses of Linear Transformations. Pitman, London, 1979.

[34] Keiiti Aki and Paul G. Richards. Quantitative Seismology, volume 2. W. H. Freeman and Co., San Francisco, CA, 1980.

[35] H. L. Van Trees. Detection, Estimation, and Modulation Theory. John Wiley and Sons, New York, 1968. 
[36] J. F. Lynch, D. K. Schwartz, and K. Sivaprasad. On the use of focused horizontal arrays as mode separation and source location devices in ocean acoustics. Part II: Theoretical and numerical modeling results. Journal of the Acoustical Society of America, 78(1):575-586, August 1985.

[37] G. H. Golub and C. F. Van Loan. Matrix Computations. The Johns Hopkins University Press, Baltimore and London, second edition, 1989.

[38] A. W. Drake. Fundamentals of Applied Probability Theory. McGraw-Hill, New York, NY, 1967.

[39] A. G. Voronovich, V. V. Goncharov, A. Yu. Nikol'tsev, and Yu. A. Chepurin. Comparative analysis of methods for the normal mode decomposition of a sound field in a waveguide: numerical simulation and full-scale experiment. Soviet Physical Acoustics, 38(4):365-370, July-August 1992.

[40] Don H. Johnson and Dan E. Dudgeon. Array Signal Processing: Concepts and Techniques. Prentice Hall Signal Processing Series. Prentice Hall, Englewood Cliffs, NJ, 1993.

[41] T. C. Yang. A method of range and depth estimation by modal decomposition. Journal of the Acoustical Society of America, 82(5):1736-1745, November 1987.

[42] William L. Brogan. Modern Control Theory. Prentice Hall, Englewood Cliffs, NJ, second edition, 1985.

[43] Alan S. Willsky. Recursive estimation. Supplementary Notes for 6.433, Spring 1994. MIT Dept. of Elec. Engr. and Comp. Science.

[44] L. Ljung. System Identification. Information and System Sciences Series. Prentice Hall, Englewood Cliffs, NJ, 1987.

[45] A. Parvulescu. Matched-signal "MESS" processing by the ocean. Journal of the Acoustical Society of America, 98(2):943-960, 1995. 
[46] A. Parvulescu and C. S. Clay. Reproducibility of signal transmissions in the ocean. The Radio and Electronic Engineer, 29(4):223 - 228, April 1965.

[47] A. Parvulescu. MESS processing. Journal of the Acoustical Society of America, 33:1674, 1961.

[48] F. DiNapoli. Personal Communication, October 1995.

[49] G. Zames. Feedback and optimal sensitivity: Model reference transformations, multiplicative seminorms, and approximate inverses. IEEE Trans. Autom. Control, AC-26:301-320, 1981.

[50] Jan Lunze. Robust Multivariable Feedback Control. Prentice Hall International Series in Systems and Control Engineering. Prentice Hall, Englewood Cliffs, NJ, 1989.

[51] Michael Grimble. Robust Industrial Control: Optimal Design Approach for Polynomial Systems. Prentice Hall International Series in Systems and Control Engineering. Prentice Hall, Englewood Cliffs, NJ, 1994.

[52] R. Headrick. Personal Communication, May 1996.

[53] Alan V. Oppenheim and Ronald W. Schafer. Discrete-time Signal Processing. Prentice Hall Signal Processing Series. Prentice Hall, Englewood Cliffs, NJ, 1989.

[54] Simon Haykin. Adaptive Filter Theory. Information and System Sciences Series. Prentice Hall, Englewood Cliffs, NJ, second edition, 1991.

[55] B. D. O. Anderson and J. B. Moore. Optimal Filtering. Information and System Sciences Series. Prentice Hall, Englewood Cliffs, NJ, 1979.

[56] J. F. Lynch, G. Jin, R. Pawlowicz, D. Ray, C.-S. Chiu, J. H. Miller, R. H. Bourke, A. R. Parsons, and R. Muench. Acoustic travel-time perturbations 
due to shallow-water internal waves and internal tides in the Barents Sea Polar Front: Theory and experiment. Journal of the Acoustical Society of America, 99(2):803-821, 1996.

[57] J. V. Candy and D. H. Chambers. Internal wave signal processing: A modelbased approach. IEEE Journal of Oceanic Engineering, 21(1):37-52, January 1996.

[58] John R. Buck, Josko Catipovic, and Hugh B. Morgenbesser, August 1993. XBT survey of south continental shelf near $41^{\circ} \mathrm{N} 71^{\circ} \mathrm{W}$ from R/V Navaho.

[59] Elvis Presley. Personal Communication, April 1995.

[60] F. B. Jensen and C. T. Tindle. Numerical modeling results for mode propagation in a wedge. Journal of the Acoustical Society of America, 82(1):211-216, July 1987.

[61] L.-L. Fu and B. Holt. Internal waves in the Gulf of California: Observations from a spaceborne radar. Journal of Geophysical Research, 89(2):2053-2060, March 1984.

[62] D. Halpern. Observations on short-period internal waves in Massachusetts Bay. Journal of Marine Research, 29(2):116-132, 1971.

[63] L. Haury, M. Briscoe, and M. Orr. Tidally generated internal wave packets in Massachusetts Bay. Nature, 278:312-317, 1979.

[64] Keith von der Heydt. Hydrophone calibration memo. Woods Hole Oceanographic Institution, February 1988.

[65] M. Grund and M. Johnson. The AMS operating system: A prototyping environment for real-time signal processing algorithm development. In Proc. of the IEEE Int. Workshop on Rapid Systems Prototyping, North Carolina, June 1995. 
[66] J. Capon. High-resolution frequency-wavenumber spectrum analysis. Proceedings of the IEEE, 57(8):1408-1418, August 1969. 